\title{
Swift and NuSTAR observations of GW170817: detection of a blue kilonova
}

\author{
P.A. Evans ${ }^{1 *}$, S.B. Cenko ${ }^{2,3}$, J.A. Kennea ${ }^{4}$, S.W.K. Emery ${ }^{5}$, N.P.M. Kuin ${ }^{5}$, O. \\ Korobkin $^{6}$, R.T. Wollaeger ${ }^{6}$, C.L. Fryer ${ }^{6}$, K.K. Madsen $^{7}$, F.A. Harrison ${ }^{7}$, Y. \\ $\mathrm{Xu}^{7}$, E. Nakar ${ }^{8}$, K. Hotokezaka ${ }^{9}$, A. Lien ${ }^{10,11}$, S. Campana ${ }^{12}$, S.R. Oates ${ }^{13}$, E. \\ Troja $^{2,14}$, A.A. Breeveld ${ }^{5}$, F. E. Marshall ${ }^{2}$, S.D. Barthelmy ${ }^{2}$, A. P. Beardmore ${ }^{1}$, \\ D.N. Burrows ${ }^{4}$, G. Cusumano ${ }^{15}$, A. D’Aì ${ }^{15}$, P. D’Avanzo ${ }^{12}$, V. D’Elia ${ }^{16,17}$, M. \\ de Pasquale ${ }^{18}$, W.P. Even ${ }^{6,19}$, C.J. Fontes ${ }^{6}$, K. Forster ${ }^{7}$, J. Garcia ${ }^{7}$, P. Giommi ${ }^{17}$, \\ B. Grefenstette ${ }^{7}$, C. Gronwall ${ }^{4,20}$, D.H. Hartmann ${ }^{21}$, M. Heida ${ }^{7}$, A.L. Hungerford ${ }^{6}$, \\ M.M. Kasliwal ${ }^{22}$, H.A. Krimm ${ }^{23,24}$, A.J. Levan ${ }^{13}$, D. Malesani ${ }^{25}$, A. Melandri ${ }^{12}$, \\ H. Miyasaka ${ }^{7}$, J.A. Nousek ${ }^{4}$, P.T. O’Brien ${ }^{1}$, J.P. Osborne ${ }^{1}$, C. Pagani ${ }^{1}$, K.L. \\ Page $^{1}$, D.M. Palmer ${ }^{26}$, M. Perri ${ }^{16,17}$, S. Pike ${ }^{7}$, J.L. Racusin ${ }^{2}$, S. Rosswog ${ }^{27}$, \\ M.H. Siegel ${ }^{4}$, T. Sakamoto ${ }^{28}$, B. Sbarufatti ${ }^{4}$, G. Tagliaferri ${ }^{12}$, N.R. Tanvir ${ }^{1}$, A. \\ Tohuvavohu ${ }^{4}$
}

\footnotetext{
${ }^{1}$ University of Leicester, X-ray and Observational Astronomy Research Group, Leicester Institute for Space and Earth Observation, Department of Physics \& Astronomy, University Road, Leicester, LE1 7RH, UK

2 Astrophysics Science Division, NASA Goddard Space Flight Center, Greenbelt MD, 20771 USA

3 Joint Space-Science Institute, University of Maryland, College Park, MD 20742, USA

${ }^{4}$ Department of Astronomy and Astrophysics, The Pennsylvania State University, University Park, PA 16802, USA

${ }^{5}$ University College London, Mullard Space Science Laboratory, Holmbury St. Mary, Dorking, RH5 6NT, U.K.

${ }^{6}$ Center for Theoretical Astrophysics, Los Alamos National Laboratory, Los Alamos, NM 87545 USA

${ }^{7}$ Cahill Center for Astronomy and Astrophysics, California Institute of Technology, 1200 East California Boulevard, Pasadena, CA 91125, U

${ }^{8}$ The Raymond and Beverly Sackler School of Physics and Astronomy, Tel Aviv University, Tel Aviv 69978, Israel

${ }^{9}$ Center for Computational Astrophysics, Simons Foundation, 162 5th Ave, New York, 10010, NY, USA

${ }^{10}$ Center for Research and Exploration in Space Science and Technology (CRESST) and NASA Goddard Space Flight Center, Greenbelt MD, 20771 USA

${ }^{11}$ Department of Physics, University of Maryland, Baltimore County, 1000 Hilltop Circle, Baltimore, MD 21250, USA

12 INAF - Osservatorio Astronomico di Brera, Via Bianchi 46, I-23807 Merate, Italy

${ }^{13}$ Department of Physics, University of Warwick, Coventry, CV4 7AL, UK
} 
14 Department of Physics and Astronomy, University of Maryland, College Park, MD 20742-4111, USA

15 INAF - IASF Palermo, via Ugo La Malfa 153, I-90146, Palermo, Italy

16 INAF-Osservatorio Astronomico di Roma, via Frascati 33, I-00040 Monteporzio Catone, Italy

17 Space Science Data Center (SSDC) - Agenzia Spaziale Italiana (ASI), I-00133 Roma, Italy

18 Department of Astronomy and Space Sciences, University of Istanbul, Beyzit 34119, Istanbul, Turkey

19 Southern Utah University, Cedar City, UT 84720, USA

${ }^{20}$ Institute for Gravitation and the Cosmos, The Pennsylvania State University, University Park, PA 16802

${ }^{21}$ Department of Physics and Astronomy, Clemson University, Kinard Lab of Physics, USA

22 Division of Physics, Mathematics and Astronomy, California Institute of Technology, Pasadena, CA 91125, USA

${ }^{23}$ Universities Space Research Association, 7178 Columbia Gateway Dr, Columbia, MD 21046, USA

24 National Science Foundation, 2415 Eisenhower Avenue, Alexandria, VA 22314, USA

25 Dark Cosmology Centre, Niels Bohr Institute, University of Copenhagen, Juliane Maries Vej 30, DK-2100 Copenhagen $\varnothing$, Denmark

${ }^{26}$ Los Alamos National Laboratory, B244, Los Alamos, NM, 87545, USA

27 The Oskar Klein Centre, Department of Astronomy, AlbaNova, Stockholm University, SE-106 91 Stockholm, Sweden

${ }^{28}$ Department of Physics and Mathematics, Aoyama Gakuin University, Sagamihara, Kanagawa, 252-5258, Japan

*To whom correspondence should be addressed; E-mail: pae9@leicester.ac.uk

With the first direct detection of merging black holes in 2015, the era of gravitational wave (GW) astrophysics began. A complete picture of compact object mergers, however, requires the detection of an electromagnetic (EM) counterpart. We report ultraviolet (UV) and X-ray observations by Swift and the Nuclear Spectroscopic Telescope ARray (NuSTAR) of the EM counterpart of the binary neutron star merger GW 170817. The bright, rapidly fading ultraviolet emission indicates a high mass $(\approx 0.03$ solar masses $)$ wind-driven outflow with moderate electron fraction $\left(Y_{e} \approx 0.27\right)$. Combined with the $\mathbf{X}$-ray limits, we favor an observer viewing angle of $\approx 30^{\circ}$ away from the orbital rotation axis, which avoids both obscuration from the heaviest elements in the orbital plane and a direct view of any ultra-relativistic, highly collimated ejecta (a gamma-ray burst afterglow).

\section{One-sentence summary}

We report X-ray and UV observations of the first binary neutron star merger detected via gravitational waves.

\section{Main Text}

At 12:41:04.45 on 2017 August 17 (UT times are used throughout this work), the Laser Interferometric GravitationalWave Observatory (LIGO) and Virgo Consortium (LVC) registered a strong gravitational wave (GW) signal (LVC trigger G298048; (1)), later named GW 170817 (2). Unlike previous GW sources reported by LIGO, which involved only black holes (3), the gravitational strain waveforms indicated a merger of two neutron stars. Binary neutron star mergers have long been considered a promising candidate for the detection of an electromagnetic counterpart associated with a gravitational wave source.

Two seconds later, the Gamma-Ray Burst Monitor (GBM) on the Fermi spacecraft triggered on a short (duration $\approx 2 \mathrm{~s}$ ) gamma-ray signal consistent with the $\mathrm{GW}$ localization, GRB 170817A $(4,5)$. The location of the 


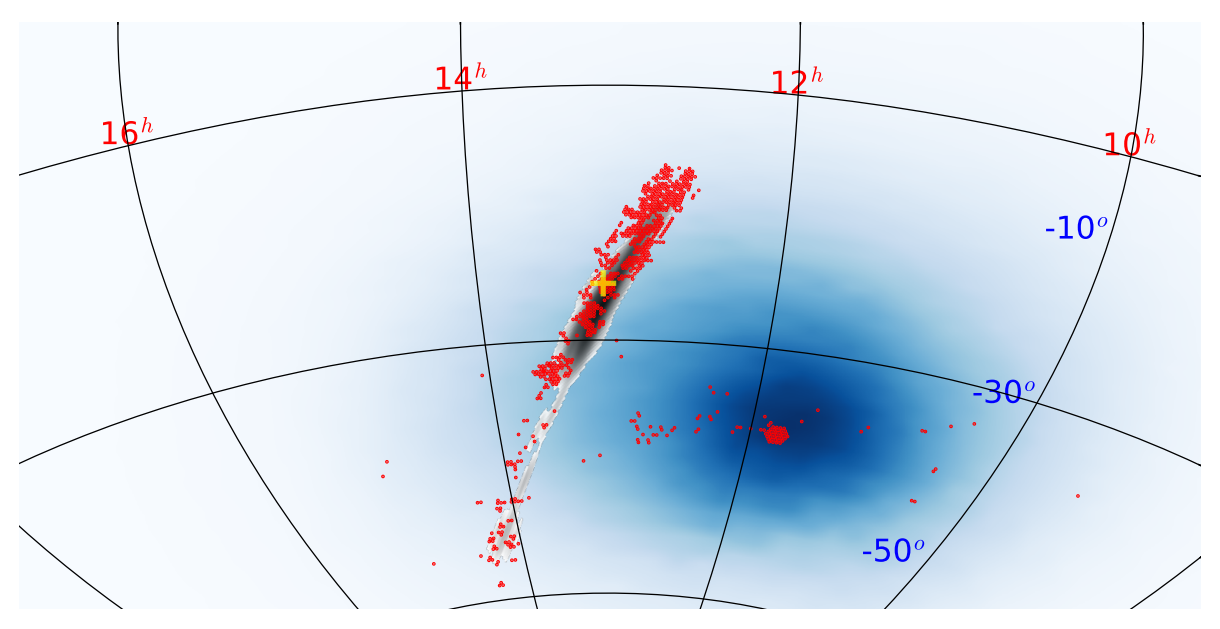

Figure 1: Skymap of Swift XRT observations, in equatorial (J2000) coordinates. The grey probability area is the GW localization (13), the blue region shows the Fermi-GBM localization, and the red circles are Swift-XRT fields of view. UVOT fields are colocated with a field of view $60 \%$ of the XRT. The location of the counterpart, EM 170817, is marked with a large yellow cross. The early 37-point mosaic can be seen, centred on the GBM probability. The widely scattered points are from the first uploaded observing plan, which was based on the singledetector GW skymap. The final observed plan was based on the first 3-detector map (11), however we show here the higher-quality map (13) so that our coverage can be compared to the final probability map (which was not available at the time of our planning; (7)).

Swift satellite (6) in its low-Earth orbit meant that the GW and gamma-ray burst (GRB) localizations were occulted by the Earth (7) and so not visible to its Burst Alert Telescope (BAT). These discoveries triggered a world-wide effort to find, localize and characterize the EM counterpart (8). We present UV and X-ray observations conducted as part of this campaign; companion papers describe synergistic efforts at radio (9) and optical/near-infrared (10) wavelengths.

\section{Search for a UV and X-ray Counterpart}

Swift began searching for a counterpart to GW 170817 with its X-ray Telescope (XRT) and UV/Optical Telescope (UVOT) at 13:37 (time since the GW and GRB triggers, $\Delta t=0.039 \mathrm{~d}$ ). At the time, the most precise localization was from the Fermi-GBM (90\% containment area of $1626 \mathrm{deg}^{2}$ ), so we imaged a mosaic with radius $\sim 1.1^{\circ}$ centered on the most probable GBM position. Subsequently at 17:54 ( $\Delta t=0.2 \mathrm{~d})$ a more precise localization became available from the LIGO and Virgo GW detectors, with a 90\% containment area of only $33.6 \mathrm{deg}^{2}$ (11). Following the strategy outlined in (12), Swift began a series of short (120 s) exposures centered on known galaxies in the GW localization (Figure 1; (7)).

No new, bright (X-ray flux, $f_{X} \geq 10^{-12} \mathrm{erg} \mathrm{cm}^{-2} \mathrm{~s}^{-1}$ ) X-ray sources were detected in the wide-area search (XRT imaged $92 \%$ of the distance-weighted GW localization (7)). In order to quantify the likelihood of recovering any rapidly fading X-ray emission, we simulated 10,000 short GRB afterglows based on a flux-limited sample of short GRBs (14), and randomly placed them in the 3D (distance plus sky position) GW localization, weighted by the GW probability. We find that in $65 \%$ of these simulations we could recover an X-ray afterglow with our 

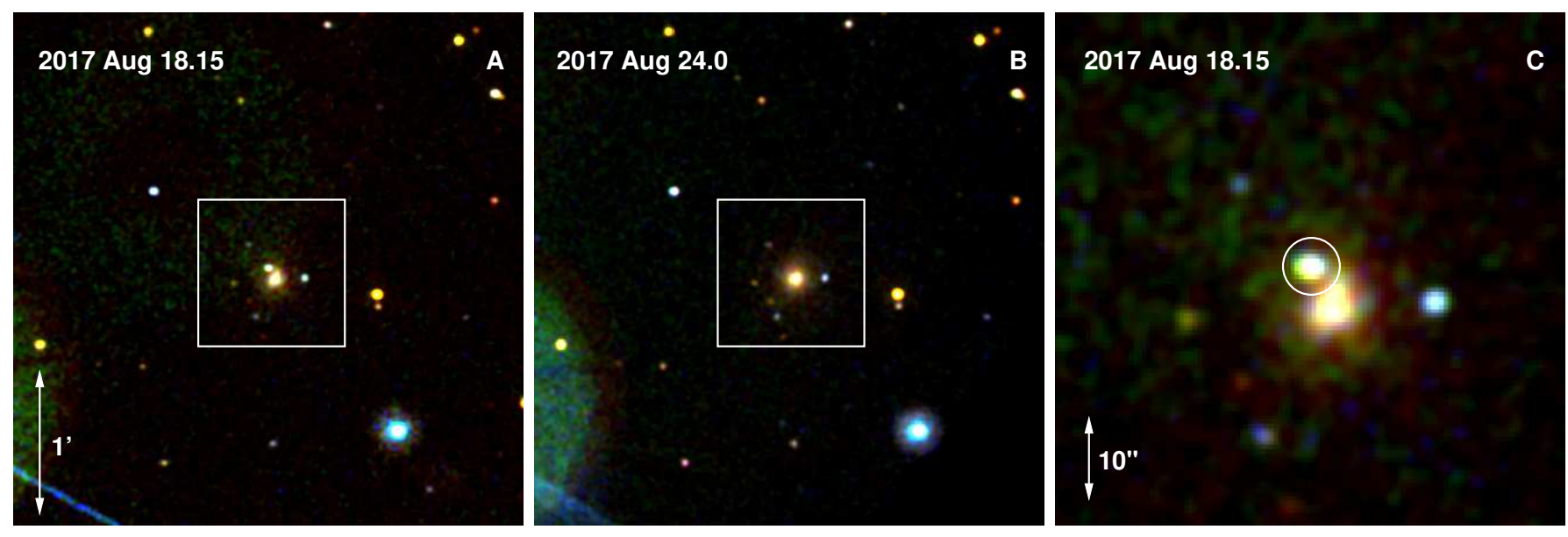

Figure 2: False-color UV image of the field of EM 170817. The $u, u v w 1$ and $u v m 2$ filters have been assigned to the red, green and blue channels respectively. Bright UV emission is clearly detected in our first epoch (panel A), which rapidly fades at blue wavelengths (panel B). Panel C shows a zoom in of the first epoch with the transient circled. All images are oriented with North up and East to the left.

wide-area tiling observations (7).

At 01:05 on 2017 August 18 ( $\Delta t=0.5 \mathrm{~d})$, a candidate optical counterpart, Swope Supernova Survey 17a (SSS17a) $(15,16)$, was reported in the galaxy NGC 4993 (distance $d \approx 40 \mathrm{Mpc}$ ). Ultimately this source, which we refer to as EM 170817, was confirmed as the electromagnetic counterpart to the GW detection and the Fermi GRB (8), making it the closest known short GRB to Earth. Follow-up observations of EM 170817 (7) with Swift began at 03:34 ( $\Delta t=0.6 \mathrm{~d}$ ) and with the Nuclear Spectroscopic Telescope ARray (NuSTAR) (17) at 05:25 ( $\Delta t=0.7 \mathrm{~d})$.

In the first exposures $(\Delta t=0.6 \mathrm{~d})$, the UVOT detected a bright fading UV source at the location of EM 170817 (Figure 2). The initial magnitude was $u=18.19_{-0.08}^{+0.09} \mathrm{mag}(\mathrm{AB})$, but subsequent exposures revealed rapid fading at UV wavelengths. The rapid decline in the UV is in contrast to the optical and near-infrared emission, which remained flat for a much longer period of time (Figure 3 , (10)).

Neither the Swift-XRT nor NuSTAR instruments detected X-ray emission at the location of EM 170817. A full listing of the Swift-XRT and NuSTAR upper limits at this location is provided in Table S2.

\section{The UV Counterpart Rules Out an On-Axis Afterglow}

In the standard model of GRBs $(20,21)$, the prompt $\gamma$-ray emission is generated by internal processes in a highly collimated, ultra-relativistic jet. As the ejecta expand and shock heat the circumburst medium, electrons are accelerated and emit a broadband synchrotron afterglow. Our UV and X-ray observations place strong constraints on the presence and/or orientation of such ejecta following GW 170817.

In Figure 4 we plot the median and 25-75\% distribution of short GRB afterglows (14), scaled to the distance of NGC 4993. While a handful of short GRBs have extremely fast-fading afterglows (22) which would have been missed by our observations, the bulk of the population would have been easily detectable (7).

We can translate these $\mathrm{X}$-ray upper limits to physical constraints using the standard analytic afterglow formulation for synchrotron emission (7). We find that for on-axis viewing geometries, our non-detections limit the amount 

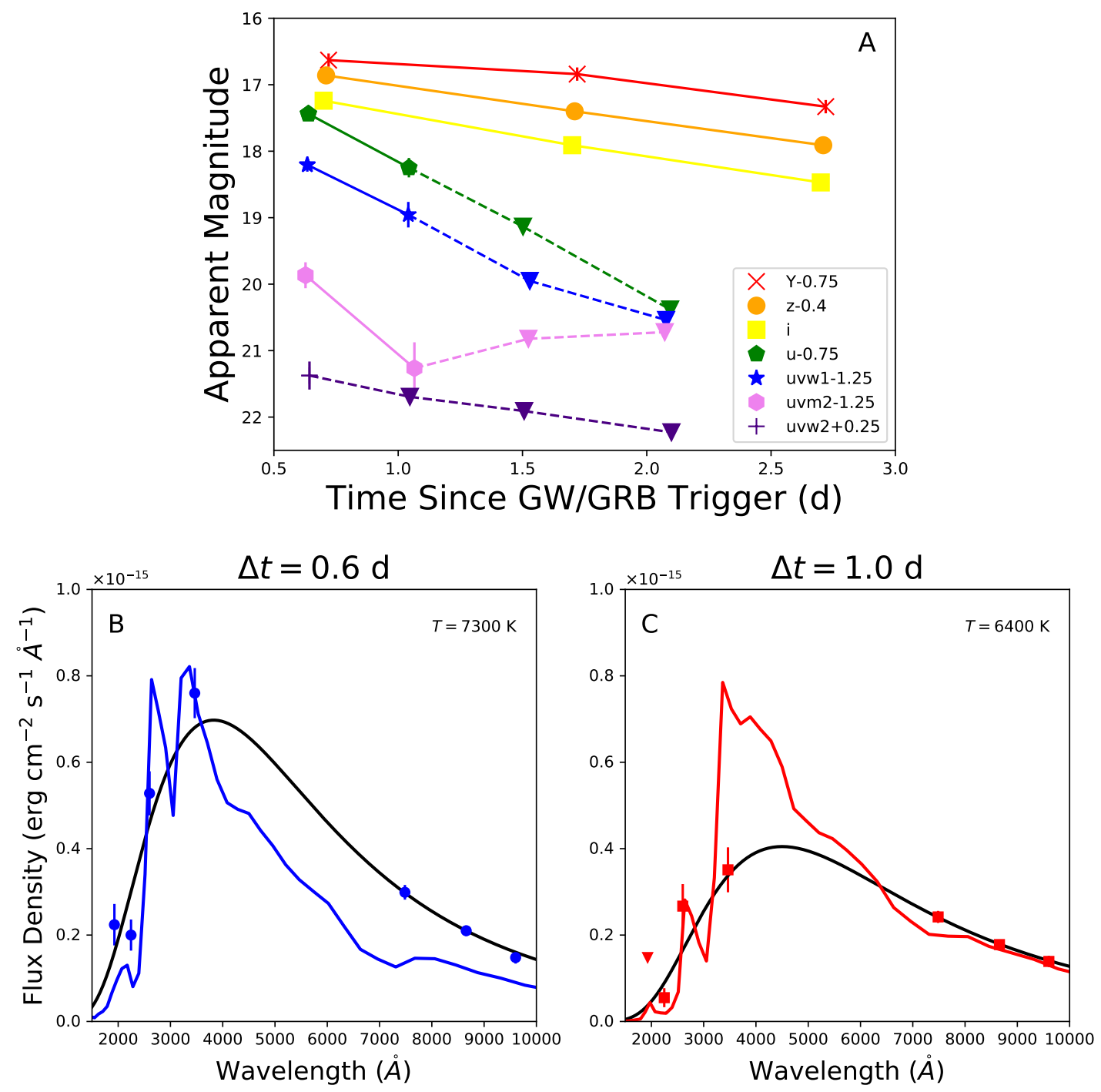

Figure 3: UV and optical light curves and SEDs. A: Swift-UVOT light curve of the optical counterpart EM 170817 of GW 170817. The data are corrected for host galaxy contamination. Upper limits are plotted as inverted triangles. Also shown are host-subtracted optical and near-infrared photometry from Pan-STARRS (18). $B$ - $C$ The spectral energy distribution of EM 170817, with blackbody models (black curves) demonstrating the rapid cooling of the ejecta. Overplotted are the best fitting kilonova models (colored lines), where the wind ejecta have mass $0.03 M_{\odot}$, and velocity $0.08 c$, while the dynamical ejecta have mass $0.013 M_{\odot}$ and velocity $0.3 c(7)$. The red triangle in the right hand figure is a $3-\sigma$ upper limit. 

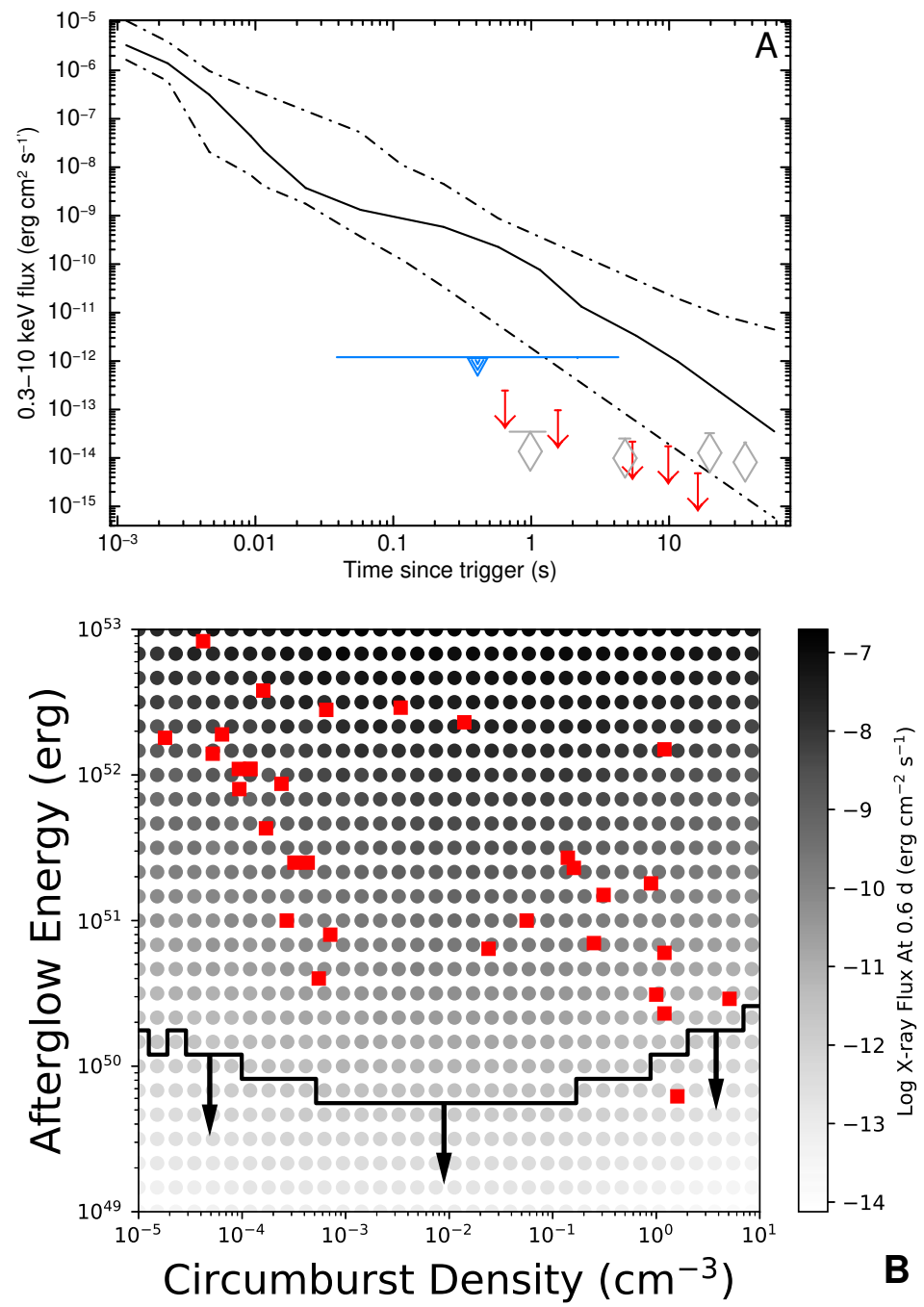

Figure 4: Predicted X-ray flux of an afterglow to GW 170817. A: The distribution of short GRB light curves (14), scaled to $40 \mathrm{Mpc}$. The solid line shows the median behavior; the two dashed lines represent the 25 and 75 percentiles. The blue line with the triangle corresponds to the time range covered by the large-scale tiling with Swift-XRT and shows the typical sensitivity achieved per tile. The red arrows represent the XRT upper limits on emission from EM 170817 obtained by summing all the data up to the time of the arrow. The grey diamonds show the NUSTAR limits on emission from EM 170817. B: The X-ray flux predicted for an on-axis jet for a range of isotropic afterglow energies and circumburst densities. The black line indicates the flux upper limit of the first NuSTAR observation; red squares are known short GRBs with $E_{\mathrm{AG}}$ and $n_{0}(19)$. Our observations rule out an energetic, ultra-relativistic outflow with $E_{\mathrm{AG}}>\sim 10^{50} \mathrm{erg}$ for on-axis geometries. 
of energy coupled to relativistic ejecta $\left(E_{\mathrm{AG}}\right)$ to be $E_{\mathrm{AG}}<\sim 10^{50} \mathrm{erg}$ (assuming the energy is radiated isotropically). To verify this result, we ran a series of simulations using the afterglow light curve code boxfit (23). Over the range of circumburst densities and afterglow energies inferred for short GRBs (19), we calculated the X-ray flux at the time of our first NUSTAR epoch (which provides the tightest constraints, given typical afterglow decay rates). The results are shown in Figure 4 yielding a similar constraint $\left(<\sim 10^{50} \mathrm{erg}\right)$ on the afterglow energy as our analytic approach.

Our X-ray upper limits also help to rule out an afterglow origin for the UV emission: the optical to X-ray spectral index $\beta_{O X} \geq 1.6$ at $\Delta t=0.6 \mathrm{~d}$, is highly inconsistent with observed GRB afterglows (24). Analysis of the UV/optical spectral energy distribution (SED) at early times ( $\Delta t \leq 2 \mathrm{~d}$ ) further supports this conclusion (7). Fitting the SED with a blackbody function yields a temperature: $T_{\mathrm{BB}}(\Delta t=0.6 \mathrm{~d})=7300 \pm 200 \mathrm{~K}$, and $T_{\mathrm{BB}}(\Delta t=1.0 \mathrm{~d}$ ) $=6400 \pm 200 \mathrm{~K}$ (Figure 33. A power-law model, as would be expected for synchrotron afterglow radiation, provides a very poor fit to the data (7). We therefore conclude that the observed UV counterpart must arise from a different physical process than an on-axis GRB afterglow.

Given the apparent absence of energetic, ultra-relativistic material along the line of sight, the detection of a short GRB is somewhat puzzling. The isotropic gamma-ray energy release of GRB $170817 \mathrm{~A}, E_{\gamma \text {,iso }}=(3.08 \pm$ $0.72) \times 10^{46} \mathrm{erg}$, is several orders of magnitude below any known short GRB (25). But even using the observed correlation (14) between $E_{\gamma \text {,iso }}$ and X-ray afterglow luminosity, the predicted X-ray flux at $\Delta t=0.6 \mathrm{~d}$ is still above our Swift and NuSTAR upper limits.

This requires an alternative explanation for the observed $\gamma$-ray emission, such as a (typical) short GRB viewed (slightly) off-axis, or the emission from a cocoon formed by the interaction of a jet with the merger ejecta (26-28). We return to this issue below in the context of late-time ( $\Delta t>\sim 10 \mathrm{~d}) \mathrm{X}$-ray emission (see also (10) and (9)).

\section{Implications of the Early UV Emission}

While inconsistent with ultra-relativistic ejecta (e.g. a GRB afterglow), our UVOT observations nonetheless imply an ejecta velocity that is a substantial fraction of the speed of light. If we convert the effective radii derived in our SED fits (Figure 3 ) to average velocities, $\bar{v} \equiv R_{\mathrm{BB}} / \Delta t$ ( $R_{\mathrm{BB}}$ is the radius of the emitting photosphere, $\Delta t$ is the time delay between the trigger and the SED), we find that $\bar{v}(\Delta t=0.6 \mathrm{~d}) \approx 0.3 c$, and $\bar{v}(\Delta t=1.0 \mathrm{~d}) \approx 0.2 c$ $(29,30)$. These velocities are much larger than seen in even the fastest known supernova explosions (31). Similarly, the rapid cooling of the ejecta, resulting in extremely red colors at $\Delta t \geq 1 \mathrm{~d}$ (Figure 3), is unlike the evolution of any common class of extragalactic transient (32).

Both of these properties are broadly consistent with theoretical predictions for electromagnetic counterparts to binary neutron star mergers known as kilonovae (sometimes called macronovae or mini supernovae) $(33,34)$. Numerical simulations of binary neutron star mergers imply that these systems can eject $\sim 10^{-3}-10^{-2}$ solar masses $\left(M_{\odot}\right)$ of material with $v \sim 0.1-0.2 c$, either via tidal stripping and hydrodynamics at the moment of contact (hereafter referred to as dynamical ejecta (35)), or by a variety of processes after the merger, which include viscous, magnetic or neutrino-driven outflows from a hyper-massive neutron star (if this is at least the temporary post-merger remnant) and accretion disc (36-39). All of these post-merger outflows are expected to have a less neutron rich composition than the dynamical ejecta and in this study we use the general term winds to refer to them collectively.

Next we examine the implications of the relatively bright UV emission at early times. Such UV emission is not a generic prediction of all kilonova models: large opacity in the ejecta due to numerous atomic transitions 
of lanthanide elements can suppress UV emission, even at early times $(40,41)$. This is particularly true for the dynamical ejecta, where a large fraction of the matter is thought to be neutron rich $\left(Y_{e} \leq 0.2\right)$ and so produces high atomic number elements (with $\sim 126$ neutrons) via rapid neutron capture (the $r$-process, (42)).

In contrast to the dynamical ejecta, a wind can have a significantly larger electron fraction, particularly if irradiated by neutrinos. $Y_{e}$ values of $\sim 0.2$ have been inferred from accretion discs around rapidly spinning black holes (43), while a long-lived hyper-massive neutron star may increase the neutrino flux even further $\left(Y_{e} \sim 0.3\right.$; (37)). As a result of these large electron fractions, nucleosynthesis is expected to stop at the second or even first $r$-process peak (elements with 82 or 50 neutrons respectively), resulting in few (if any) lanthanide elements and a dramatically reduced opacity.

Our X-ray non-detections place limits on the presence of a long-lived hyper-massive neutron star (7). In particular, we can rule out any plausible neutron star remnant with a strong magnetic field that lived past the time of our first Swift and NuSTAR observations (which would effectively be a stable remnant, given the viscous time scale of the accretion disc). Nonetheless, a short-lived or low-magnetic field hyper-massive neutron star, or a rapidly spinning black hole would both be consistent with our results.

To investigate the plausibility of a wind origin for the early UV emission, we have produced a series of 2-D models, varying the ejecta properties (mass, velocity, composition; $(7,44)$ ). We assume that the tidal ejecta are more neutron rich $\left(Y_{e} \approx 0.04\right)$ than the wind ejecta $\left(Y_{e} \approx 0.27-0.37\right)$, and produce a sizable fraction of lanthanides that obscure the optical and UV emission. The spatial distribution of this high-opacity ejecta is based on merger models (45). Obscuration by the disc formed from this high-opacity material causes a viewing-angle effect (44).

In order to reproduce the early UV emission, we require models with a wind ejecta mass $>\sim 0.03 M_{\odot}$. Furthermore, a modest electron fraction $\left(Y_{e} \approx 0.27\right)$, with significant amounts of elements from the first $r$-process peak, is strongly favored over larger $Y_{e}$ ejecta ( $Y_{e} \approx 0.37$, corresponding to mostly Fe-peak elements).

The presence of bright UV emission strongly constrains the observer viewing angle of the binary neutron star merger. Sight lines in the plane of the merger are expected to exhibit dramatically reduced UV emission due to the presence of the Lanthanide-rich dynamical ejecta. For a wind mass $\left(M_{\text {wind }}\right) \approx 0.03 M_{\odot}$, a viewing angle of $\theta_{\text {obs }}<\sim 30^{\circ}$ with respect to the rotation axis is preferred. Orientations up to $\sim 40^{\circ}$ can be accommodated with $M_{\text {wind }} \approx 0.1 M_{\odot}$; at larger viewing angles the wind ejecta mass becomes unphysically large.

While the wind component can provide a good fit to the UV emission, on its own it under-predicts the observed optical/near-infrared flux at this time. Adding dynamical ejecta with $M_{\text {dyn }} \approx 0.01 M_{\odot}$ and $v \approx 0.3 c$ can provide a reasonable fit to the early SEDs (Figure 33. However, we emphasize that the properties of the dynamical ejecta are only poorly constrained at early times; analysis of the full optical/near-infrared light curve is necessary for accurate constraints on the Lanthanide-rich material (e.g. (10)).

While much of the gamma-ray emission generated during the $r$-process is re-radiated at optical/near-infrared wavelengths, it may also be possible to observe directly emission lines from $\beta$-decay in the NuSTAR bandpass. We have calculated the expected signal from 10-100 keV for a range of ejecta masses, and it is well below the NuSTAR limits for GW 170817 (7).

The above modeling of the kilonova emission assumes that the merger ejecta is unaffected by any energetic jet (or that no such jet is formed). For jets with a narrow opening angle $\left(\theta_{\text {jet }}<10^{\circ}\right)$, numerical simulations (26) have shown that any such jet-ejecta interaction will have negligible effects on the observed light curves on the time scales probed by our observations.

However, if the jet opening angle were sufficiently large, the energy from this jet (and the resulting cocoon) may accelerate material in the merger ejecta to mildly relativistic velocities. Numerical simulations in our companion 
paper (10) offer some support for this scenario, providing a reasonable fit to the temperature and bolometric luminosity evolution of EM 170817. However, they lack the detailed radiation transport calculations presented here.

\section{Late-Time X-ray Emission: Off-Axis Jet or Cocoon}

While no X-ray emission at the location of EM 170817 was detected by Swift or NuSTAR, a faint X-ray source was detected by Chandra at $\Delta t \approx 9 \mathrm{~d}(46)$, although the flux was not reported. Subsequent Chandra observations at $\Delta t \approx 15 \mathrm{~d}$ reported $L_{X} \approx 9 \times 10^{38} \mathrm{erg} \mathrm{s}^{-1}(47,48)$. A variety of models predict long-lived X-ray emission at a level $>\sim 10^{40} \mathrm{erg} \mathrm{s}^{-1}$ following the merger of two neutron stars. For example, (quasi-)isotropic X-ray emission may be expected due to prolonged accretion onto a black hole remnant, or from the spin-down power of a longlived hyper-massive neutron star. These models are not consistent with the Swift or NuSTAR limits, or the Chandra flux (7), suggesting that, if a magnetar formed after the merger event, it collapsed to a black hole before our first $\mathrm{X}$-ray observation (i.e. within $0.6 \mathrm{~d}$ of formation).

A possible explanation for the late-time X-ray emission is an off-axis (orphan) afterglow (49). If the binary neutron star merger produces a collimated, ultra-relativistic jet, initially no emission will be visible to observers outside the jet opening angle. As the outflow decelerates, the relativistic beaming becomes weaker and the jet spreads laterally, illuminating an increasing fraction of the sky. Off-axis observers can expect to see rising emission until the full extent of the jet is visible, at which point the decay will appear similar to that measured by on-axis observers. Simulations of such events showed that starting a few days after the merger, off-axis afterglows represent the dominant population of GW counterparts detectable by Swift (50).

We ran a series of simulations using the boxfit code (23) to utilize our X-ray limits and the reported Chandra detections to constrain the orientation of GW 170817 (7). For the median values of short GRB afterglow energy, $E_{\mathrm{AG}}=2 \times 10^{51} \mathrm{erg}$, circumburst density, $n_{0}=5 \times 10^{-3} \mathrm{~cm}^{-3}$, and jet opening angle, $\theta_{\text {jet }}=0.2$ radian $\left(12^{\circ} ;(19)\right)$, the resulting light curves are plotted in Fig. 5. With the Swift and NuSTAR non-detections, these models rule out any viewing angle with $\theta_{\mathrm{obs}}<\sim 20^{\circ}$. Assuming the emission reported by Chandra results from an orphan afterglow, we infer $\theta_{\text {obs }} \approx 30^{\circ}$.

This inferred orientation is entirely consistent with the results of our analysis of the early UV emission. However, it is difficult to simultaneously explain the observed gamma-ray emission in this scenario, as it would require a viewing angle only slightly outside the jet edge (10). Either the observed GRB 170817A is powered by a source distinct from this jet, or we are forced to disfavor an orphan afterglow model for the late-time X-ray emission.

Alternatively, delayed X-ray emission may result if the initial outflow speed is mildly relativistic, as would be expected from models where propagation in the merger ejecta forms a hot cocoon around the jet (26). In this case the rise is dictated by the time necessary for the cocoon to sweep up enough material in the circumburst medium to radiate efficiently; this in turn depends on the energy carried by the expanding cocoon, its bulk Lorentz factor and the circumburst density. Figure 6 6 shows X-ray light curves predicted by this model for a range of plausible values of these parameters, along with the X-ray limits from NuSTAR and Swift-XRT and the Chandra detection $(47,48)$. The latest NUSTAR datapoint disfavors energetic cocoon models, particularly those at high density. But lower energy or density models can fit all the X-ray data, whilst simultaneously accounting for the gamma-ray emission (10).

Our inferences regarding the origin of the late-time X-ray emission are broadly consistent with the conclusions reached in our companion radio paper (9). Both an orphan afterglow and a mildly relativistic cocoon model make specific predictions for the evolution of the broadband flux over the upcoming months after the merger (Figures5. 


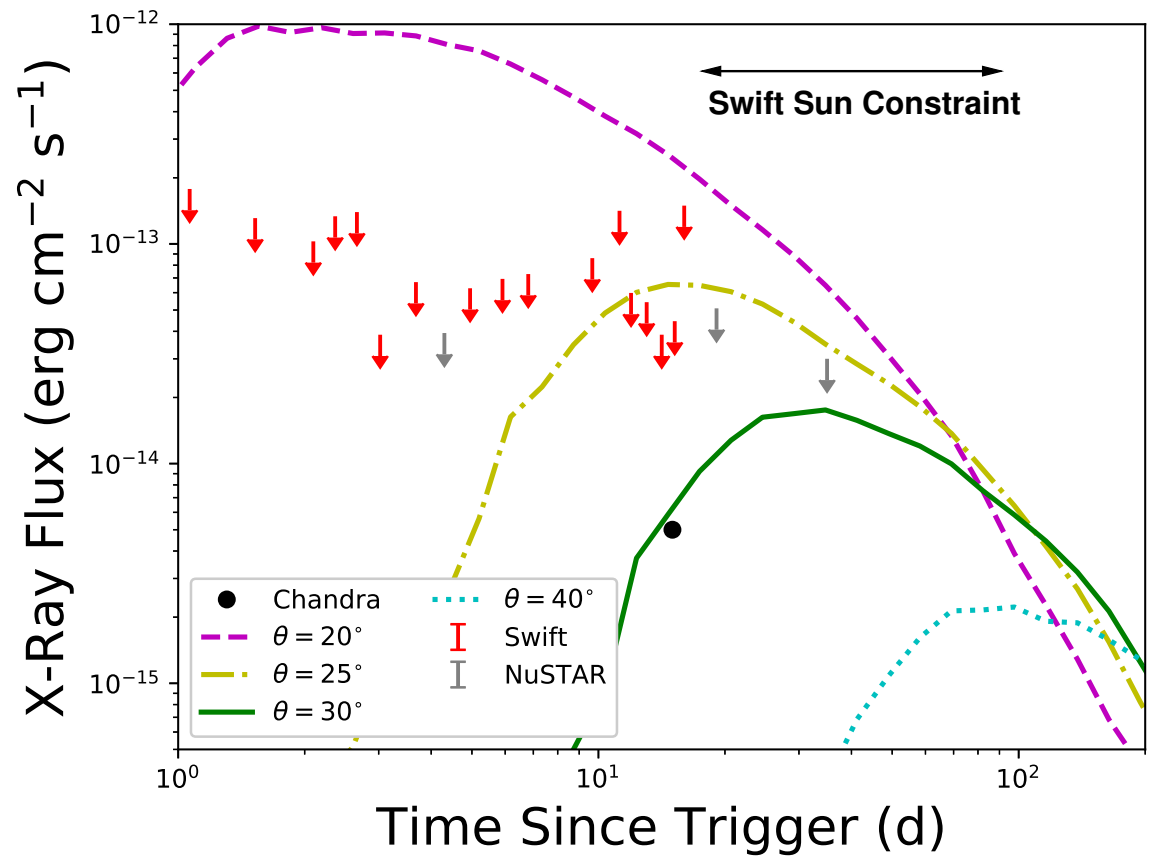

Figure 5: Simulated X-ray afterglow light curves for typical short GRB parameters (23). Here, $E_{\mathrm{AG}}=$ $2 \times 10^{51} \mathrm{erg}, n_{0}=5 \times 10^{-3} \mathrm{~cm}^{-3}$ ), and $\theta_{\text {jet }}=0.2$ radian; the true values of these parameters are uncertain and vary between GRBs. Curves are shown for a range of viewing angles, with the Swift-XRT and NuSTAR limits marked. An off-axis orientation of $\approx 30^{\circ}$ is consistent with both the early Swift-XRT and NuSTAR limits, and the recently reported Chandra detection (46). The anticipated peak time will occur when Swift and Chandra cannot observe the field due to proximity to the Sun. 


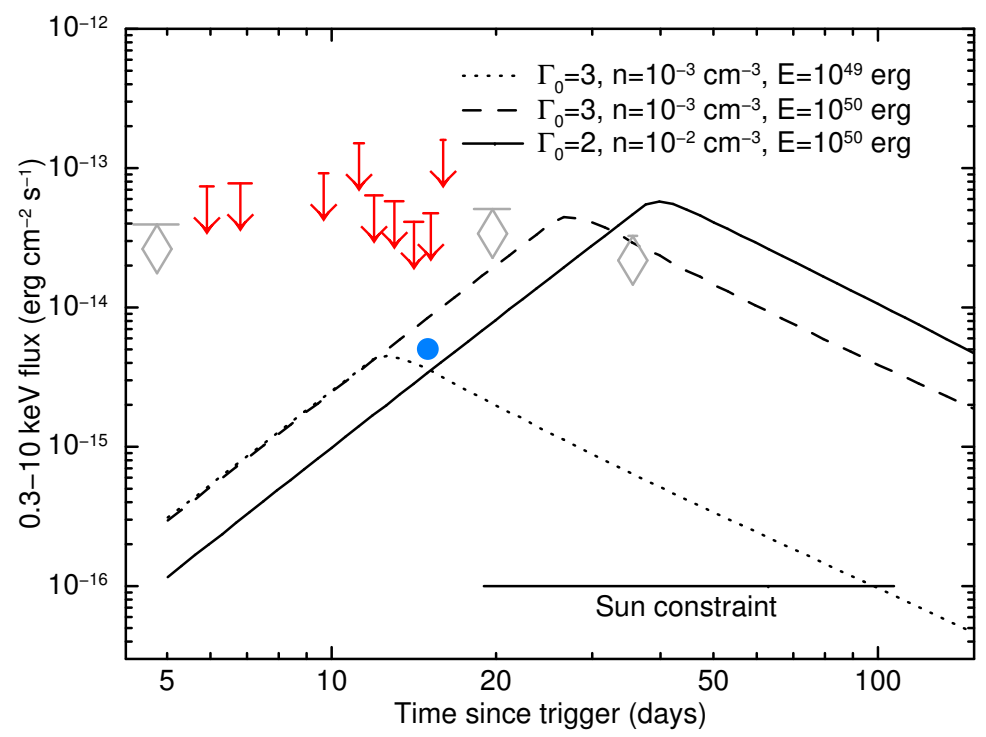

Figure 6: Predicted X-ray light curves from a mildly relativistic jet. The jet is based on model predictions (26), for a range of different values for the inital bulk Lorentz factor of the cocoon $\left(\Gamma_{0}\right)$, circumburst density $(n)$ and cocoon energy (E). Data points are the Swift-XRT (red arrows) and NuSTAR (grey diamonds) upper limits and the Chandra detection (blue) of EM 170817. The range of plausible peak times is not observable by Swift (or Chandra). 
6. (9)).

\section{Conclusions}

The discovery of a short GRB simultaneous with a GW binary neutron star merger represents the start of a new era of multi-messenger astronomy. It confirms that binary neutron star mergers can generate short gamma-ray transients (51), though the connection to classical short GRBs remains unclear. Furthermore, GW 170817 provides robust evidence that $r$-process nucleosynthesis occurs in the aftermath of a binary neutron star merger $(10)$.

While a kilonova detection following a short GRB has been previously reported $(52,53)$, our multi-wavelength dataset has allowed us confront kilonova models with UV and X-ray observations. The absence of X-ray emission largely rules out the presence of an energetic, ultra-relativistic, and collimated outflow viewed from within the opening angle of the jet. The late-time X-ray emission is consistent with a collimated, ultra-relativistic outflow viewed at an off-axis angle of $\approx 30^{\circ}$ (i.e. an orphan afterglow). A mildly relativistic outflow, as may be expected if the jet were enveloped by a hot cocoon, is also consistent with our X-ray data (and may naturally explain the peculiar properties of the gamma-ray emission; (10)).

The presence of bright, rapidly fading UV emission was not a generic prediction of kilonova models and requires special circumstances to avoid obscuration by the heavy elements formed in the dynamical ejecta. We find that we can reproduce the early UV and optical emission with a massive $\left(M \approx 0.03 M_{\odot}\right)$ and high-velocity $\left(v \approx 0.08 c\right.$ ) outflow comprised of moderate- $Y_{e}$ (first $r$-process peak) material at a viewing angle of $\approx 30^{\circ}$; such winds may be expected if the remnant is a relatively long-lived hyper-massive neutron star or a rapidly spinning black hole. Alternatively, if the hot cocoon is able to accelerate material in the ejecta to mildly relativistic speeds, this may also be able to account for the early UV emission (10).

\section{Acknowledgments}

This paper is dedicated to the memory of Neil Gehrels (1952-2017), who was PI of Swift until his untimely death in February 2017. Neil's inspirational leadership was the lifeblood of Swift and he was always keen to innovate and expand the mission's capabilities. Without his direction, leadership and support, the work we present here would not have been possible.

Funding for the Swift mission in the UK is provided by the UK Space Agency. SRO gratefully acknowledges the support of the Leverhulme Trust Early Career Fellowship (SRO). The Swift team at the MOC at Penn State acknowledges support from NASA contract NAS5-00136. The Italian Swift team acknowledge support from ASIINAF grant I/004/11/3. SR has been supported by the Swedish Research Council (VR) under grant number 201603657_3, by the Swedish National Space Board under grant number Dnr. 107/16 and by the research environment grant "Gravitational Radiation and Electromagnetic Astrophysical Transients (GREAT)" funded by the Swedish Research council (VR) under Dnr 2016- 06012. This research used resources provided by the Los Alamos National Laboratory Institutional Computing Program, which is supported by the U.S. Department of Energy National Nuclear Security Administration under Contract No. DE-AC52-06NA25396. VLT data were obtained under ESO programme number 099.D-0668. NuSTAR acknowledges funding from NASA Contract No. NNG08FD60C.

The observations are archived at http://www.swift.ac.uk for Swift and https://heasarc.gsfc.nasa.gov/docs/nustar/nustar_archive.html for NuSTAR under the observation IDs given in Table S2. Reduced photometry and surveyed areas are tabulated in the supplementary material. The BOXFIT software is 
available at http://cosmo.nyu.edu/afterglowlibrary/boxfit2011.html, SUPERNu at https://bitbucket.org/drrossum/supernu/wiki/Home, Access to WINNET source code and input files will be granted upon request via: https://bitbucket.org/korobkin/winnet. The dynamical model ejecta are available via http://compactmerger.astro.su.se/downloads_fluid_trajectories.html (run 12) The SUPERNU and BOXFIX input files are available as Supplementary Files at Science Online. 


\section{Supplementary material for Swift and NuSTAR observations of GW170817: detection of a blue kilonova}

\section{Materials and Methods}

\section{Observations}

Advanced LIGO and advanced Virgo (operating as the LIGO/Virgo Consortium: hereafter 'LVC') registered GW 170817 at 12:41:04.45 UT on 2017 August 17. This was announced to the followup community first as an LVC/Gamma-ray Coordinates Network (GCN) notice at 13:08 UT, and then by (1), (trigger 'G298048'). Fermi Gamma-ray Burst Monitor (GBM) triggered on a weak short gamma-ray signal at 12:41:06 UT (4), reported as a short GRB (sGRB, (54)). A weak signal was also observed by the INTEGRAL satellite (55). At this time, the Swift Burst Alert Telescope (BAT; (56)) was pointing at RA, Dec $(\mathrm{J} 2000)=02^{h} 24^{m} 18.0^{s},-52^{\circ} 17^{\prime} 13^{\prime \prime}$, i.e. away from the GBM and GW position. Indeed, the GW localization region was entirely occulted by the Earth (Figure [S1], with none of the probability region above the Earth limb (defined as $69^{\circ}$ from the center of the Earth, which accounts for the radius of the Earth plus $100 \mathrm{~km}$ of atmosphere, up to the Kármán line, which commonly represents the top of the atmosphere). The position of EM 170817A (below) was likewise not visible to Swift at the trigger time, while the very edge of the GBM error region overlapped the edge of the BAT field of view $(0.1 \%$ of the GBM probability was within the BAT field of view). We found no evidence for a signal in the BAT within 100-s of the GW and GBM triggers.

Swift observations comprising more than one pointing can only be uploaded to the spacecraft in the form of a pre-planned science timeline which requires a ground-station pass. In order to obtain some rapid coverage, we uploaded commands using the NASA Tracking and Data Relay Satellite System (TDRSS) network to utilize the on-board 37-point tiling pattern. Using this pattern the X-ray Telescope (XRT; (57)) covered a region $\sim 1.1^{\circ}$ in radius, which was centered on the GBM position. The UV/optical Telescope (UVOT; (58)) has a smaller field of view $\left(17^{\prime} \times 17^{\prime}\right)$ than the XRT $\left(24.6^{\prime}\right.$ diameter), thus there are gaps between the tiles in UVOT. These observations began at 13:37 UT, $0.04 \mathrm{~d}$ after GW 170817.

The initial GW skymap was created using only a single GW detector (LIGO-Hanford; (59)) and thus covered almost the entire sky. The GBM error had a statistical position error of $11.6^{\circ}$ radius $(1-\sigma)$ with a systematic error estimated to be at least $3.7^{\circ}(4)$; the GBM localization probability has a $90 \%$ confidence area of $1626 \mathrm{deg}^{2}$. We convolved the GW skymap with the GWGC catalog (60), using the 3D information in the GW skymap and the methodology described by (50). This effectively re-weights the GW probability according to our knowledge of the location of the stellar mass in the local Universe, accounting for our estimate of how incomplete that knowledge is. We next multiplied the resultant probability map by a map of the GBM localization probability and renormalised the result, then identified the most probable XRT fields-of-view from this skymap. Swift-XRT and UVOT observations of these fields, with a nominal $120 \mathrm{~s}$ per exposure began at 17:16 UT, $0.19 \mathrm{~d}$ after GW 170817. The onboard slewing algorithm is not perfectly modelled, thus exposure times vary someone from the nominal value.

At $\sim 17: 30$ UT a revised GW skymap was released which used data from all three LVC interferometers and had a $90 \%$ credible region of $31 \operatorname{deg}^{2}(11)$. Since this error region only overlapped the outer contours of the GBM 


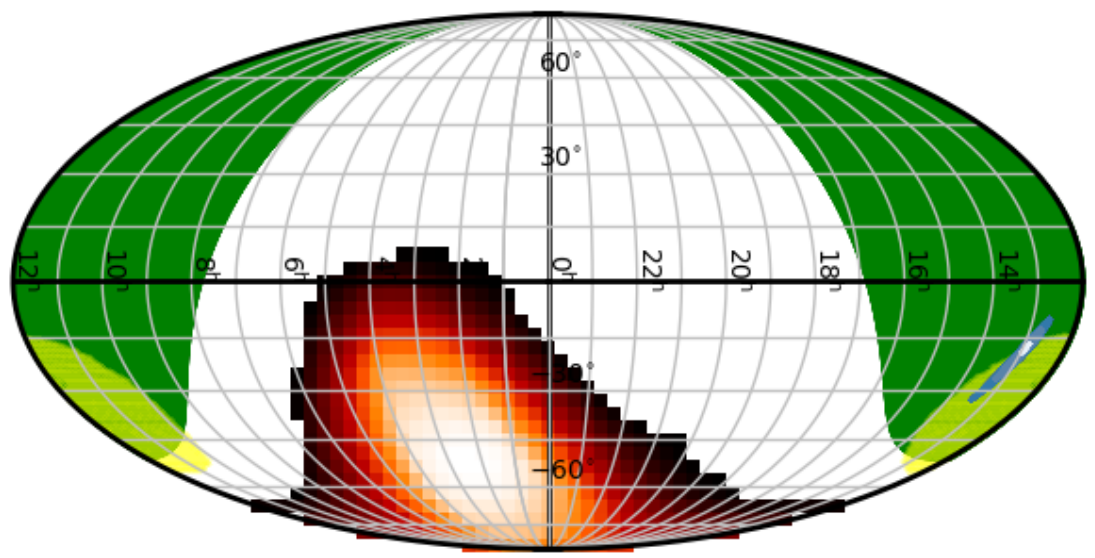

Figure S1: The BAT field of view at the time of the GW/GRB trigger. The black/red/white scale indicates the BAT coding fraction; the darker colour indicates lower partial coding fraction (i.e. more toward the edge of the field of view). The GW localizations probability contours are plotted in blue. The green region shows the part of the sky occulted by the Earth and the yellow region is the Fermi-GBM 90\% confidence region.

error region, our planned observations did not significantly cover this revised localisation. We therefore convolved this new skymap with GWGC as above, and generated a new observing plan based on this probability map; these observations began at 20:24 UT, $0.31 \mathrm{~d}$ after GW 170817, and are listed in Table S3.

At 01:05 UT on 2017 August 18, (15) reported the detection of a candidate EM counterpart, hereafter denoted 'EM 170817', apparently associated with the galaxy NGC 4993 (distance $\approx 40$ Mpc (61)). Shortly thereafter, several others groups reported detection of the source $(62,63)$, sometimes with differing names (e.g. SSS17a, DLT17ck). For simplicity we refer to the object henceforth as EM 170817.

Swift-XRT and UVOT observed this field for $2 \mathrm{ks}$, starting on 2017 Aug 18 at 03:35 UT ( $\Delta t=0.6 \mathrm{~d}$ ), temporarily overriding the wide-area search of the GW error region. The optical source was detected in all UV filters, but no X-ray counterpart was seen (64).

The nature of EM 170817 was not clear at this time, so Swift resumed the wide-area search of the GW error region, but regularly interrupted it to carry out repeat observations of EM 170817. These targeted observations continued after the large-area tiling was complete (at $\Delta t=4.3 \mathrm{~d}$ ), apart from the interval 22:04 on August 24 to 22:14 on August 26, at which times the source was too close to the Moon for Swift observations.

After the discovery of X-ray emission from EM 170817 by Chandra (46-48) we increased the frequency of these observations, until the field entered the Swift Sun constraint at 05:46 on September 2, from which it will emerge on December 02. Details of the Swift observations of EM 170817 are given in Table S2

The Nuclear Spectroscopic Telescope ARray (NuSTAR) (17) also observed the location of EM 170817, taking data at four different epochs starting at $4.8 \mathrm{~d}$ after the GW trigger. Details of these observations are also presented in Table $\mathbf{S 2}$ 


\section{Data analysis}

\subsection{Swift-BAT}

While the location of EM 170817A was not visible to Swift at the time of the trigger, the initial GW localisation (which covered almost the entire sky) and the edge of the Fermi localization error were within the BAT field of view. We therefore searched the raw $15-350 \mathrm{keV}$ BAT light curves for any evidence for GRB emission within 100 -s of the GW/GBM trigger. No such emission was detected. As there was no BAT trigger, this search is limited to the raw 15-350 keV light curves (the so-called 'rate data' accumulated onboard), which have time bins of 64ms, $1 \mathrm{~s}$ and $1.6 \mathrm{~s}$. Assuming a sGRB with a typical spectrum in the BAT energy range (i.e. a simple power-law model with a power-law index of -1.32 , from the averaged sGRB power-law indices based on (65)), the 5- $\sigma$ upper limit in the 1.6-s binned light curve corresponds to a $15-350 \mathrm{keV}$ flux of $<2.3 \times 10^{-8} \mathrm{erg} \mathrm{cm}^{-2} \mathrm{~s}^{-1}$ for an on-axis energy response of the BAT, and $<4.8 \times 10^{-7} \mathrm{erg} \mathrm{cm}^{-2} \mathrm{~s}^{-1}$ for an extremely off-axis (i.e within the Fermi error region) energy response of the BAT.

Swift began observations of EM 170817 with the UVOT at 03:37 on 2017 August 18 ( $\Delta t=0.6 \mathrm{~d}$ ), initially with just the $u v w 2$, uvm2, uvw1, and $u$ filters. Observations with the full 6-filter complement (including the $v$ and $b$ bands) began at $\Delta t=2.5 \mathrm{~d}$ using a pointing offset (required to avoid to a nearby bright star). The source was detected in all UV filters; $b$ and $v$ band yield only marginal detections or upper limits, in part due to the considerable galaxy background. UVOT analysis was carried out using HEASOFT v6.21 (66) and UVOT CALDB version 20170130 (67).

Absolute photometry was obtained by extracting the photon counts using a circular aperture of $3^{\prime \prime}$ radius centered on EM 170817, followed by an aperture correction to the calibration standard of $5^{\prime \prime}$. The use of this smaller aperture reduced the contamination from NGC 4993 and increased the signal-to-noise ratio (68). Background counts were extracted from a region located well outside of the host galaxy. Count rates (including coincidence loss correction) were obtained using the HEASOFT tool UVOTSOURCE.

To determine the host galaxy contribution to the source, we measured the background-subtracted count rate in the source region in the interval $\Delta t=9.5-16 \mathrm{~d}$, by which time the source had faded below detectability in all filters. A few images were removed due to an unstable spacecraft attitude. The host count rate was then subtracted from the earlier measurements of EM 170817; this was performed for each filter and exposure individually. The host-subtracted count rates were then converted to $\mathrm{AB}$ magnitudes using zero points from (69).

Examination of the spectral energy distribution (SED) derived from the photometry showed that the flux drops off steeply going further into the UV. As a result the effective wavelength of the UV bands is shifted to the red. We calculated the effective wavelengths of the UVOT bands by iteratively computing the weighted average of the wavelength multiplied by the effective area multiplied by the approximate flux (as derived from the SED), normalized by the effective area and flux integral. The results are shown in Table S1.

Finally, light curves were corrected for Galactic extinction, $E(B-V)=0.106$ (70), derived using a region of size $2^{\circ}$. The final, background-subtracted light curves are shown in Figure 2A.

\subsection{Swift-XRT}

The data were analysed using HEASOFT v6.21 (66) and CALDB version 20170501 (67). The XRT data were grouped into "analysis blocks" of overlapping fields, to a maximum size of $\sim 50^{\prime}$ in diameter. Source detection was carried out on each of these blocks using the iterative method developed by (71). Any sources found were ranked according to their likelihood of being a transient related to the GW trigger. The method and ranking is 
Table S1: Swift-UVOT photometry of EM 170817. The reported magnitudes have been corrected for host contamination but not for Galactic extinction. Upper limits are quoted at the $3 \sigma$ level. Epochs lacking sufficient SED constraints (and thus lacking estimates of the effective wavelength) are indicated with "...".

\begin{tabular}{llllll} 
Filter & $\begin{array}{l}\lambda_{\text {eff }} \\
(A)\end{array}$ & $\begin{array}{l}\Delta t \\
(\mathrm{~d})\end{array}$ & $\begin{array}{l}\text { Duration } \\
(\mathrm{d})\end{array}$ & $\begin{array}{l}\text { Count Rate } \\
\left(\mathrm{s}^{-1}\right)\end{array}$ & AB Magnitude \\
\hline \hline uvw2 & 2296 & 0.6431 & 0.0029 & $0.151 \pm 0.032$ & $21.13_{-0.21}^{+0.26}$ \\
uvw2 & 2366 & 1.0469 & 0.0029 & $0.041 \pm 0.024$ & $>21.45$ \\
uvw2 & $\cdots$ & 1.5067 & 0.003 & $0.026 \pm 0.022$ & $>21.66$ \\
uvw2 & $\cdots$ & 2.0992 & 0.0646 & $0.027 \pm 0.014$ & $>21.98$ \\
uvw2 & $\cdots$ & 2.3612 & 0.0702 & $0.021 \pm 0.013$ & $>22.16$ \\
uvw2 & $\cdots$ & 2.6601 & 0.0376 & $0.013 \pm 0.014$ & $>22.21$ \\
uvw2 & $\cdots$ & 3.0286 & 0.196 & $0.0195 \pm 0.0088$ & $>22.42$ \\
\hline uvm2 & 2365 & 0.6272 & 0.0043 & $0.092 \pm 0.018$ & $21.12_{-0.20}^{+0.24}$ \\
uvm2 & 2405 & 1.0652 & 0.0308 & $0.026 \pm 0.011$ & $22.52_{-0.39}^{+0.62}$ \\
uvm2 & $\cdots$ & 1.5237 & 0.0378 & $0.0122 \pm 0.0087$ & $>22.07$ \\
uvm2 & $\cdots$ & 2.0723 & 0.0336 & $0.012 \pm 0.010$ & $>21.97$ \\
uvm2 & $\cdots$ & 4.9533 & 0.4697 & $0.0062 \pm 0.0068$ & $>22.47$ \\
\hline uvw1 & 2590 & 0.6344 & 0.0029 & $0.641 \pm 0.064$ & $19.46_{-0.10}^{+0.11}$ \\
uvw1 & 2790 & 1.0407 & 0.0016 & $0.321 \pm 0.062$ & $20.21_{-0.19}^{+0.23}$ \\
uvw1 & $\cdots$ & 1.5293 & 0.0345 & $0.042 \pm 0.029$ & $>21.20$ \\
uvw1 & $\cdots$ & 2.0777 & 0.0350 & $0.004 \pm 0.023$ & $>21.79$ \\
uvw1 & $\cdots$ & 3.0211 & 0.1960 & $0.004 \pm 0.018$ & $>22.05$ \\
\hline u & 3529 & 0.6387 & 0.0014 & $2.93 \pm 0.22$ & $18.19_{-0.08}^{+0.09}$ \\
u & 3666 & 1.0431 & 0.0008 & $1.39 \pm 0.20$ & $19.00_{-0.15}^{+0.17}$ \\
u & $\cdots$ & 1.5021 & 0.0015 & $0.27 \pm 0.11$ & $20.79_{-0.31}^{+0.61}$ \\
u & $\cdots$ & 2.3546 & 0.0674 & $0.098 \pm 0.094$ & $>20.41$ \\
u & $\cdots$ & 4.9461 & 0.4673 & $0.038 \pm 0.072$ & $>20.85$ \\
\hline b & $\cdots$ & 2.3565 & 0.0674 & $0.27 \pm 0.16$ & $>19.31$ \\
b & $\cdots$ & 2.6554 & 0.0348 & $0.42 \pm 0.19$ & $19.93_{-0.40}^{+0.65}$ \\
b & $\cdots$ & 3.0258 & 0.1951 & $0.15 \pm 0.12$ & $>19.71$ \\
b & $\cdots$ & 3.6517 & 0.1006 & $0.26 \pm 0.15$ & $>19.37$ \\
b & $\cdots$ & 5.9098 & 0.1654 & $0.22 \pm 0.14$ & $>19.50$ \\
\hline v & $\cdots$ & 2.3668 & 0.0683 & $0.11 \pm 0.12$ & $>18.72$ \\
v & $\cdots$ & 2.6657 & 0.0357 & $0.12 \pm 0.12$ & $>18.67$ \\
v & $\cdots$ & 3.0029 & 0.1628 & $0.172 \pm 0.097$ & $>18.72$ \\
v & $\cdots$ & 3.6620 & 0.1016 & $0.07 \pm 0.10$ & $>18.95$ \\
v & $\cdots$ & 5.9161 & 0.1641 & $0.19 \pm 0.12$ & $>18.54$ \\
\hline & & & & & \\
\hline
\end{tabular}


described in detail by (72). No sources above rank 3 (i.e. uncataloged, but below catalog limits) were found in our search of the GW error region.

We focus only on the data at the location of EM 170817. No source was found in the first 2-ks observation of this source (64), with a $0.3-10 \mathrm{keV} \mathrm{3- \sigma}$ upper limit of $5.5 \times 10^{-3} \mathrm{ct} \mathrm{s}^{-1}$. Assuming an energy conversion of $4 \times 10^{-11} \mathrm{erg} \mathrm{cm} \mathrm{s}^{-1} \mathrm{ct}^{-1}$, which is typical for GRB afterglows (73), this corresponds to a flux of $2.2 \times 10^{-13} \mathrm{erg}$ $\mathrm{cm}^{-2} \mathrm{~s}^{-1}$. Given the distance to NGC 4993 of $\sim 40 \mathrm{Mpc}$ this equates to $L_{\mathrm{X}}<4.2 \times 10^{40} \mathrm{erg} \mathrm{s}^{-1}$.

No X-ray emission was detected near the position of EM 170817 in the next four observations, when a standard GRB afterglow would be at its brightest (see, Figure 4 and (50), their figures 12-13). However, we did detect a source $\sim 2$ days after the trigger, once we had accumulated $17.5 \mathrm{ks}$ of exposure time with XRT (74). The source was poorly localized due to its faintness, but lay $11.3^{\prime \prime}$ from the optical counterpart position, and $8.1^{\prime \prime}$ from NGC 4993, with a position uncertainty of $6.4^{\prime \prime}$ (90\% confidence). Summing all of the X-ray data on this source, and correcting the astrometry by aligning the X-ray sources in the field with objects cataloged in 2MASS as described by (71) (and using only XRT sources detected with flags 'Good' or 'Reasonable', i.e. unlikely to be spurious detections.), we obtain a final position of RA, Dec $(\mathrm{J} 2000)=13^{\mathrm{h}} 09^{\mathrm{m}} 47.65^{\mathrm{s}},-23^{\circ} 23^{\prime} 01.6^{\prime \prime}$, with an uncertainty of $3.9^{\prime \prime}$ (radius, $90 \%$ confidence). This is $10.2^{\prime \prime}$ from the position of EM 170817, and 0.9" from the position of NGC 4993, suggesting that the emission detected is from the host galaxy, rather than the electromagnetic counterpart. This interpretation was confirmed by Chandra observations $(47,48,75,76)$. Taken with the detection of radio emission from the galaxy $(9,77-79)$ this may indicate an active galactic nucleus in NGC 4993; however the X-ray emission appears somewhat diffuse (75) and is thus likely made up of multiple X-ray emitters in that galaxy.

Since this emission may contaminate the location of EM 170817, the predicted count-rate from the host galaxy (assuming point-like emission) was taken into account when calculating upper limits. Summing all of the XRT data (172 ks), we calculate a $3-\sigma$ upper limit at the location of EM 170817 of $2.8 \times 10^{-4} \mathrm{ct} \mathrm{s}^{-1}$. Using the same assumptions as above, this corresponds to $L_{\mathrm{X}}<2.1 \times 10^{39} \mathrm{erg} \mathrm{s}^{-1}(0.3-10 \mathrm{keV})$. The count rates or upper limit for the host NGC 4993 and EM 170817 for each individual observation are given in Table S2

\subsection{NUSTAR}

The NUSTAR data were reduced using NUSTARDAS version 06Dec16_01.7.1 within HEASOFT (66), and CALDB version 20170614 (67). We used all standard settings in NUPIPELINE, but included filtering modes for the South Atlantic Anomaly (SAACALC=2, SAAMODE=OPTIMIZED, TENTACLE=YES), to reduce the solar low energy background. For extracting count rates, we used an optimized extraction region of radius $20^{\prime \prime}$, which allows for a possible 1- $\sigma$ positional offset of $8^{\prime \prime}$ in the NuSTAR absolute astrometry (17), and ensures that the NUSTAR full-width half-maximum point-spread function (PSF), which has a diameter of $20^{\prime \prime}$, is included in the extraction region. The region was centered on the location of EM 170817 (15). Spectra and responses were obtained for each module and epoch using NUPRODUCTS with default settings.

We combined the data from the two NuSTAR focal plane modules (FPM): 'FPMA' and 'FPMB', and for the first epoch we obtained count rates of $7.8 \times 10^{-4} \mathrm{ct} \mathrm{s}^{-1}(3-10 \mathrm{keV}), 1.2 \times 10^{-3} \mathrm{ct} \mathrm{s}^{-1}(10-39 \mathrm{keV})$ and $7.5 \times 10^{-4}$ $\mathrm{ct} \mathrm{s}^{-1}(39-79 \mathrm{keV})$. We compared these count rates with predicted background rates obtained from NUSKYBKG, which are based on a model fitted to the background of the entire module (80), and found that they agreed at the 1- $\sigma$ level, indicating that the neither EM 170817 nor NGC 4993 were detected. We calculated the 3- $\sigma$ upper limits on emission from EM 170817 by assuming a power-law spectrum with a photon index of -2 and absorption Hydrogen column density $3 \times 10^{21} \mathrm{~cm}^{-2}$, as is typical for GRBs (73), and assuming a distance of $40 \mathrm{Mpc}$. These are given in Table S2. We analyzed the other epochs in the same way; the source was likewise undetected, with the limits given 
Table S2: X-ray pointed observations of EM 170817 with Swift-XRT and NuSTAR. For XRT we give the measured luminosity or 3- $\sigma$ upper limit of NGC 4993 and 3- $\sigma$ limit at the location of EM 170817. For NuSTAR we give only the latter.

\begin{tabular}{|c|c|c|c|c|}
\hline ObsID & $\begin{array}{l}\text { Start time }^{a} \\
\text { (UTC) }\end{array}$ & $\begin{array}{l}\text { Exposure } \\
(\mathrm{ks})\end{array}$ & $\begin{array}{c}\log L_{X, \mathrm{NGC} 4993} \\
0.3-10 \mathrm{keV} \\
\mathrm{erg} \mathrm{s}^{-1}\end{array}$ & $\begin{array}{c}\log L_{X, \text { EM } 170817} \\
(0 .) 3-10 \mathrm{keV}^{b} \\
\mathrm{erg} \mathrm{s}^{-1}\end{array}$ \\
\hline \multicolumn{5}{|l|}{ Swift } \\
\hline 07012978001 & Aug 18 at $03: 34: 33(0.62)$ & 2.00 & $<40.56$ & $<40.72$ \\
\hline 07012167001 & Aug 18 at $12: 11: 49(0.98)$ & 0.12 & $<41.88$ & $<41.70$ \\
\hline 07012978002 & Aug 18 at $13: 29: 43(1.03)$ & 1.99 & $<40.65$ & $<40.53$ \\
\hline 07012978003 & Aug 19 at $00: 18: 22(1.48)$ & 2.99 & $<40.58$ & $<40.40$ \\
\hline 07012978004 & Aug 19 at 13:24:05 (2.03) & 4.98 & $<40.22$ & $<40.29$ \\
\hline 07012979001 & Aug 19 at 18:30:52 (2.24) & 4.97 & $40.10_{-0.22}^{+0.18}$ & $<40.41$ \\
\hline 07012979002 & Aug 20 at $03: 24: 44(2.61)$ & 4.98 & $39.98_{-0.25}^{+0.20}$ & $<40.43$ \\
\hline 07012979003 & Aug 20 at $08: 28: 05$ (2.82) & 14.96 & $<40.16$ & $<39.87$ \\
\hline 07012979004 & Aug 21 at 01:43:44 (3.54) & 9.97 & $<40.20$ & $<40.11$ \\
\hline 07012979005 & Aug 22 at 00:05:57 (4.48) & 9.90 & $<40.18$ & $<40.08$ \\
\hline 07012979006 & Aug 23 at 06:22:57 (5.74) & 9.04 & $<40.21$ & $<40.12$ \\
\hline 07012979007 & Aug 23 at 23:59:57 (6.47) & 10.37 & $39.83_{-0.21}^{+0.17}$ & $<40.14$ \\
\hline 07012979008 & Aug 26 at 23:59:57 (9.47) & 7.30 & $39.80_{-0.26}^{+0.21}$ & $<40.22$ \\
\hline 07012979009 & Aug 28 at 10:46:17 (10.92) & 3.37 & $<40.35$ & $<40.43$ \\
\hline 07012979010 & Aug 29 at 01:04:57 (11.52) & 11.78 & $39.98_{-0.17}^{+0.15}$ & $<40.06$ \\
\hline 08012979011/12 & Aug 30 at 01:00:57 (12.51) & 15.81 & $39.64_{-0.23}^{+0.18}$ & $<40.02$ \\
\hline 07012979015 & Aug 31 at $02: 27: 52$ (13.57) & 34.88 & $39.90_{-0.10}^{+0.09}$ & $<39.87$ \\
\hline 08012979014/17 & Sep 01 at 05:53:04 (14.72) & 25.62 & $39.63_{-0.19}^{+0.16}$ & $<39.93$ \\
\hline 08012979016/19 & Sep 02 at $08: 40: 56(15.83)$ & 3.76 & $<40.68$ & $<40.46$ \\
\hline \multicolumn{5}{|l|}{ NuSTAR } \\
\hline 90361003002 & Aug 18 at $05: 25(0.7)$ & 23.9 & - & $<39.7$ \\
\hline 90361003004 & Aug 21 at $20: 45$ (4.3) & 40.3 & - & $<39.6$ \\
\hline 90361003005 & Sep 04 at 17:56 (18.2) & 12.6 & - & $<40.1$ \\
\hline 90361003006 & Sep 05 at 14:51 (19.1) & 19.3 & - & $<39.9$ \\
\hline 90361003007 & Sep 06 at 17:56 (20.1) & 23.1 & - & $<39.8$ \\
\hline 90361003009 & Sep 21 at $11: 10(34.9)$ & 54.1 & - & $<39.5$ \\
\hline
\end{tabular}

${ }^{a}$ Observation year is 2017, times in parentheses are days since GW 170817. ${ }^{b}$ XRT luminosities are $0.3-10 \mathrm{keV}$, NUSTAR are 3-10 keV. 
in Table S2 Combining the data from all epochs yields a limiting luminosity of $L=2.8 \times 10^{39} \mathrm{erg} \mathrm{s}^{-1}$ (3-10 $\mathrm{keV}), 1.2 \times 10^{40} \mathrm{erg} \mathrm{s}^{-1}(10-39 \mathrm{keV})$, and $1.7 \times 10^{41} \mathrm{erg} \mathrm{s}^{-1}(39-79 \mathrm{keV})$. The non-detection of NGC 4993 in the $>3 \mathrm{keV}$ band pass of NuSTAR suggests a relatively soft spectrum, given the detection by Swift-XRT. Indeed, we find no evidence for an elevated count-rate at the location of NGC 4993. Thus, while we cannot rule out that our NUSTAR limits on the flux from EM 170817 are slightly underestimated due to contamination by host-galaxy emission, any such contamination is small and will have little effect.

\section{Interpretation and discussion}

\subsection{Gamma-Ray Emission}

If the GRB 170817 was associated with GW 170817, then it is the closest sGRB with known distance: regardless of the connection with the optical counterpart, the prompt GW analysis reported a sky-averaged distance estimate of $39 \pm 17 \mathrm{Mpc}$.

Assuming a distance of $40 \mathrm{Mpc}$ (i.e. to NGC 4993; (61)), the Fermi-GBM detection corresponds to an isotropicequivalent $1 \mathrm{keV}-10 \mathrm{MeV}$ luminosity $L_{\text {iso }}=1.6( \pm 0.6) \times 10^{47} \mathrm{erg} \mathrm{s}^{-1}(25)$. This is three orders of magnitude below the lowest-energy sGRB in the sample reported by (14), consistent with the predictions of (50). That work compared the observed flux of sGRBs detected by Swift, Fermi and CGRO without known redshift with that which would have been seen from the Swift sGRBs of known redshift had they been in the range of advanced LIGO/Virgo. They predicted that a binary neutron star merger detected by advanced LIGO/Virgo would be several orders of magnitude less luminous than GRBs detected previously. Given this low luminosity, GW 170817 allows us to probe the parameter space not covered by the sGRBs detected hitherto. While the GBM spectral properties of the object $(25,81)$ are fairly typical of sGRBs, it should be borne in mind that this event is so underluminous compared to the previous sample of GRBs, that models constructed for those objects may not be simply transferrable to such a low-luminosity event as this. Indeed (see Section 3.3.2), the low-luminosity is difficult to explain as arising from an ultra-relativistic jet at all. The implications of the sub-luminous gamma-ray emission are explored in further detail in (10).

\subsection{UV/Optical Emission}

\subsubsection{SED Construction and Fitting}

Since our UVOT detections extend out to only $\Delta t=1.0 \mathrm{~d}$, we focus on the early-time behavior of the optical counterpart in this work. We constructed the spectral energy distribution (SED) of EM 170817 using data from our work and the GCN Circulars. We limit the dataset considered here to the $i z y$ photometry provided by the Pan-STARRS project $(18,82)$. At the time of the preparation of this manuscript, this was one of the few datasets with host-subtracted photometry (like the UVOT), and the transient field was previously calibrated in these filters. We linearly interpolated the Pan-STARRS 1 observations to match the two epochs of UVOT photometry.

After correcting for Galactic extinction, we fit the resulting SED to a blackbody function. Adding a free parameter for host extinction did not improve the fit quality. At $\Delta t=0.6 \mathrm{~d}$, we find $T_{\mathrm{BB}}=7300 \pm 200 \mathrm{~K}$, corresponding to $R_{\mathrm{BB}}=6.0 \times 10^{14} \mathrm{~cm}$ and $L_{\mathrm{BB}}=7 \times 10^{41} \mathrm{erg} \mathrm{s}^{-1}$. The blackbody model yields a fit statistic of $\chi_{\mathrm{r}}^{2}=2.8$ (5 degrees of freedom). At $\Delta t=1.0 \mathrm{~d}$, we derive $T_{\mathrm{BB}}=6400 \pm 200 \mathrm{~K}$, corresponding to $R_{\mathrm{BB}}=$ $6.5 \times 10^{14} \mathrm{~cm}$ and $L_{\mathrm{BB}}=5 \times 10^{41} \mathrm{erg} \mathrm{s}^{-1}$. At this epoch, we derive a goodness of fit of $\chi_{\mathrm{r}}^{2}=1.6$ (4 degrees of freedom). The resulting SED fits are plotted in Figure 2B-C. 
For comparison, we also fit the SED at both of these epochs to a power-law model (as would be expected from synchrotron afterglow emission) of the form $f_{\lambda} \propto \lambda^{-\alpha}$. At $\Delta t=0.6 \mathrm{~d}$, we find $\alpha=1.0 \pm 0.4$. The fit quality is extremely poor: $\chi_{\mathrm{r}}^{2}=102.8$ (5 degrees of freedom). Repeating this analysis at $\Delta t=1.0 \mathrm{~d}$, we find $\alpha=1.0 \pm 0.7$ and $\chi_{\mathrm{r}}^{2}=93.5$ ( 4 degrees of freedom). Clearly the power-law model provides a much worse fit to the early SED.

\subsubsection{Model Comparisons to the Early UV/Optical Light Curve}

Our kilonova calculations to model the early ( $\Delta t \leq 2 \mathrm{~d}$ ) UV/optical emission from EM 170817 are based on the approach of Wollaeger et al. (44). We employ the multigroup, multidimensional radiative Monte Carlo code SUPERNU (83-85) with the set of opacities produced by the Los Alamos suite of atomic physics codes (86-88).

We studied a range of 1-dimensional and 2-dimensional models, systematically exploring different uncertainties affecting the kilonova light curves: ejecta mass, velocity, composition, and morphology, as well as the model for the energy deposition in post-nucleosynthetic radioactive decays. Our 2-dimensional models are based on the dynamical ejecta morphologies from (45), computed by following long-term evolutions of the ejecta from neutron star mergers, which in turn were simulated in (89). The $r$-process nucleosynthesis and radioactive heating are computed using the nuclear network code WINNET (90-92). Reaction rates for nucleosynthesis are taken from the compilation of (93) for the finite range droplet model (FRDM; (94)), including density-dependent weak reaction rates $(95)$ and fission $(96,97)$. Coordinate- and time-dependent thermalization of nuclear energy is computed using empirical fits developed in (98) and (99).

The abundances of trans-lead elements depend sensitively on the nuclear mass model and, since they dominate the production of $\alpha$-particles, the production of these elements can dramatically alter the heating at late times $(98,99)$. On the other hand, the nuclear heating rate on the timescale up to one to two days is dominated by $\beta$ decay, which shows consistency across different nuclear mass models (98). Because the modeling here is focused on this early-time data, we opt to utilize the FRDM nuclear mass model and do not study the uncertainties of the nuclear heating here.

Instead, we focus on the properties of the ejecta, which consist of two components: a dynamical ejecta component and a wind ejecta component. The eight most sophisticated models in (44) have labels of the form $\gamma A_{1}, \gamma A_{2}, \gamma B_{1}, \gamma B_{2} \ldots \gamma D_{1}, \gamma D_{2}$, where the letter A-D represents the tidal morphology, are taken from (45), see also (100), their figure 1 for an illustration; the subscripted number represents the wind type (below).

The dynamical ejecta are in an axisymmetric, very neutron-rich outflow $\left(Y_{e} \approx 0.04\right)$ composed almost entirely of the main $r$-process elements with significant mass fraction of lanthanides and actinides $(\approx 14 \%)$, taken from (45). The wind ejecta are in a spherically-symmetric, moderately neutron-rich outflow with two different degrees of neutron richness: i) (subscript 1 in the model descriptor) $Y_{e}=0.37$ with a composition dominated by iron peak and slightly-above iron peak isotopes, and ii) (subscript 2 in the model descriptor) $Y_{e}=0.27$ with a composition dominated by elements at or just above the first $r$-process peak. Astrophysical and thermodynamical conditions to generate the wind compositions were sampled from typical outcomes of the high- and low-latitude wind trajectories, simulated in (101) and (38).

The $\gamma$-models in (44) take into account the distinct composition of the two outflow components, which in turn determine the opacities, ionization conditions, radioactive nuclear heating and thermalization. The " $\gamma$ " notation indicates that the gray $\gamma$-ray transport is also included to accurately account for the $\gamma$-ray energy escape or deposition.

To fit the observed data, we allowed the mass and velocity to vary for both of our two components. We also considered two different compositions for the wind ejecta based on electron fractions of 0.27 and 0.37 . The SEDs 

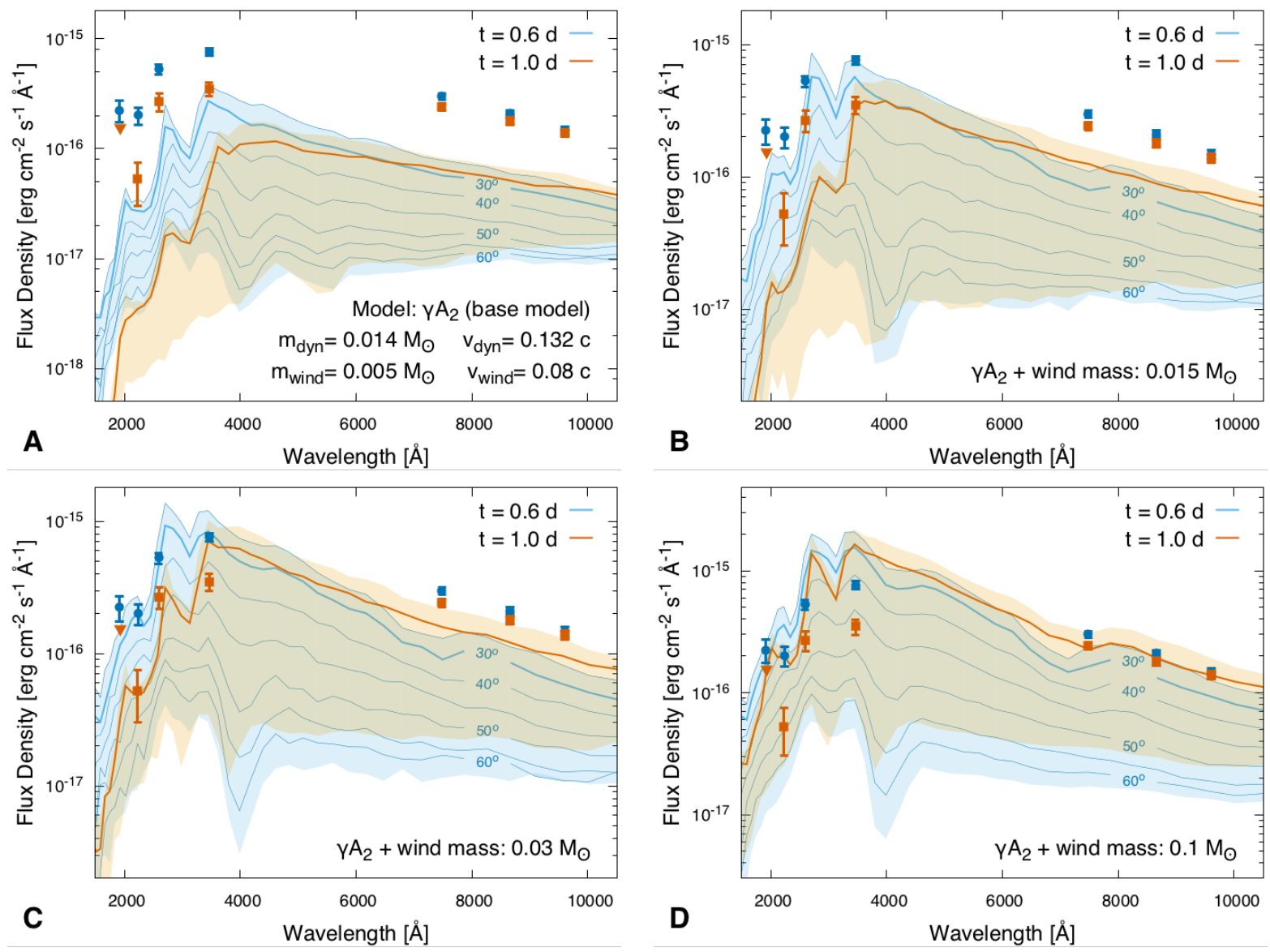

Figure S2: Model SEDs for kilonovae compared to our data. The SEDs were created for four models based on the $\gamma A_{2}$ (i.e. with the wind-2 composition), for different orientations and for two different epochs. The shaded regions display the range of model SEDs for different viewing angles over the range considered here. Thick solid lines show the flux for viewing angle $\theta \approx 30^{\circ}$, and thin lines represent adjacent angular bins with increasing viewing angle going up to $\approx 60^{\circ}$. Data points are shown as circles with error bars, or triangles (for upper limits). The models span a range of wind masses: a baseline model $\gamma A_{2}$ (panel A) has relatively low wind mass of $0.005 M_{\odot}$. In the following panels (B-D): $m_{\text {wind }}=0.015 M_{\odot}, 0.03 M_{\odot}$ and $0.1 M_{\odot}$. To fit the observed data points, the wind mass needs to be at least $0.03 M_{\odot}$. 

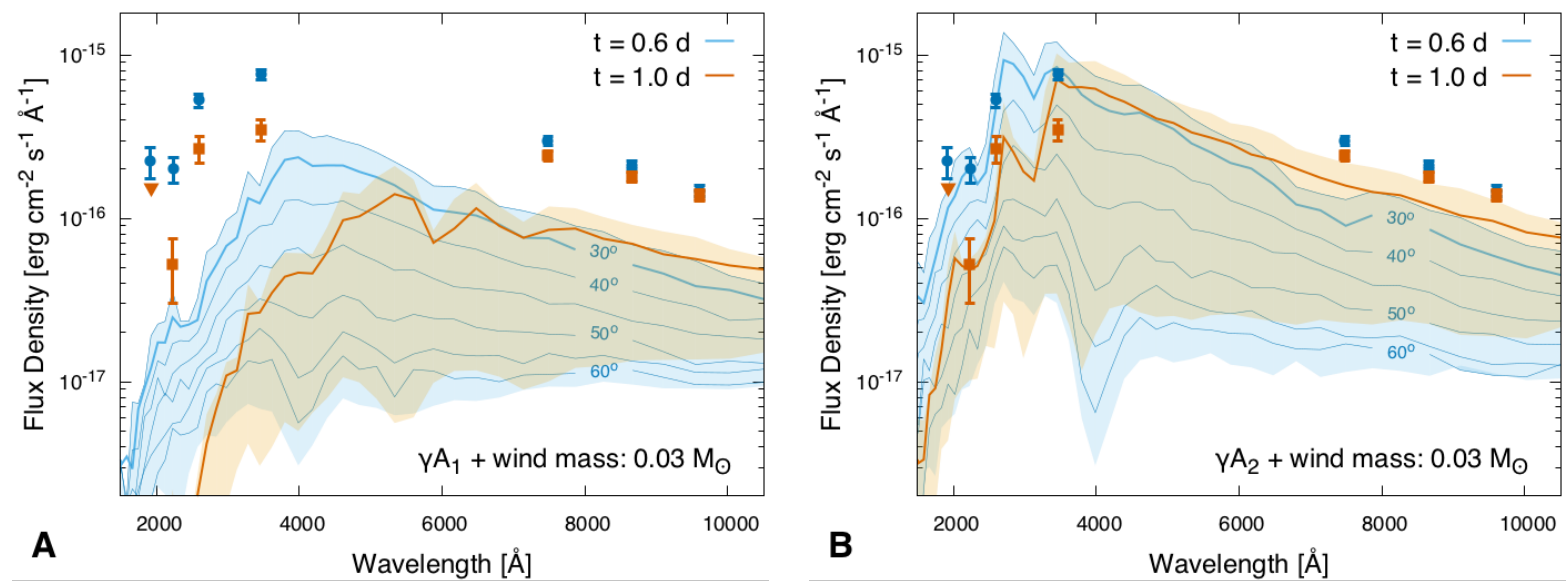

Figure S3: The effect of wind electron fraction on the SED. These SEDs have the same velocities and masses of the ejecta but different composition: "wind 1" (A) with abundant iron-group and the $d$-shell elements vs. "wind 2" (B) with the first peak elements, largely representing the $s$ - and $p$-shell elements and relatively fewer $d$-shell elements. Notation for the plots is the same as in the previous figure. The iron-group dominated composition not only exhibits lower brightness but also shows much more reddening in the spectrum between the two epochs. Datapoints are as in Figure $\mathbf{S 2}$

at times of $0.6 \mathrm{~d}$ and $1.0 \mathrm{~d}$ after the launch of the explosion are shown in Figure $\mathrm{S} 2$. This figure plots the flux as a function of wavelength for a variety of viewing angles. We focused on the dynamical ejecta configuration A, because only in this morphology the polar region is free from the "lanthanide curtain" created by the dynamical ejecta, and the wind is completely unobscured when viewed on-axis.

Figure S2 demonstrates that in order to fit the observed emission, we must raise the wind ejecta mass to at least $0.03 M_{\odot}$ (see panels $\mathrm{C}-\mathrm{D}$ ). With a wind ejecta mass of $0.1 M_{\odot}$, a fit to the data can be made with a viewing angle of $40^{\circ}$. Although this is at the high end of the predicted wind ejecta masses, it is not impossible to produce such high-mass accretion disk outflow with e.g. an asymmetric merger $(39,43,102)$. It is also possible that we are observing more irregular wind morphologies which will in any case produce brighter transients (by a factor of a few) than our spherical models.

Figure S3 compares the SEDs for wind ejecta mass $m_{\text {wind }}=0.03 M_{\odot}$ between the two different wind compositions that we explore. The high electron-fraction wind ejecta model is unable to match the data without increasing the ejecta mass further, favoring of the neutron-rich wind ejecta, which indeed is close to the values predicted for the polar regions $(37,38,102)$. The spectrum for the model with high electron-fraction exhibits much faster reddening between the epochs than its medium electron-fraction counterpart.

We emphasize that the "effective" opacity of our wind model $2\left(Y_{e}=0.27\right.$; first $r$-process peak elements) is actually somewhat lower than that of wind model 1 ( $Y_{e}=0.37$; Fe-peak elements with effective $\kappa \approx 1 \mathrm{~cm}^{2} \mathrm{~g}^{-1}$ ). This is a result of the less complex atomic configurations (44). As a result, the models with the Fe-peak composition for the disk wind become redder much more quickly and are therefore difficult to match with the bright UV emission seen at early times.

The neutron rich dynamical ejecta does not directly contribute significantly to the ultraviolet and optical emission in the first two days. In fact, the spectra and light curves in optical and UV are completely insensitive to the dynamical ejecta mass, and only weakly sensitive to its velocity in the red part of the optical spectrum. Figure $\mathbf{S 4}$ 

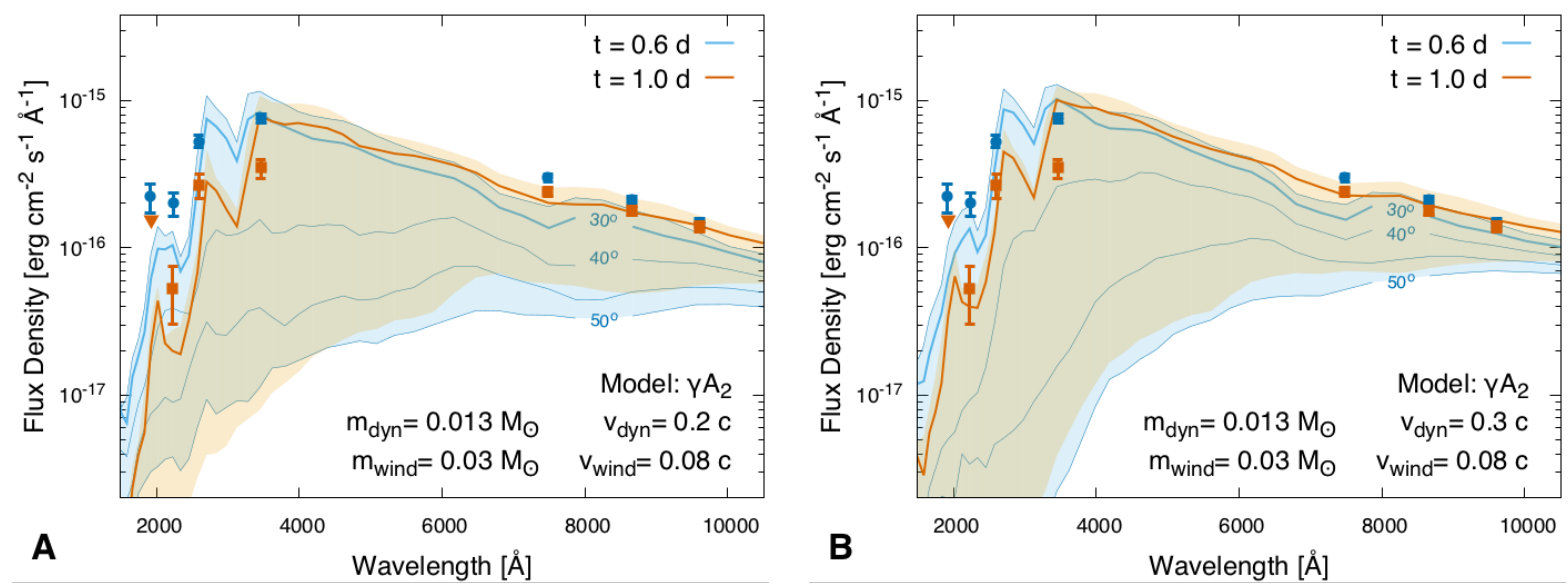

Figure S4: The effect of dynamical ejecta velocity on the SED. These SEDs have the same velocities and masses of the wind ejecta but different velocities of the dynamical ejecta: $v_{\mathrm{dyn}}=0.2 c$ (A) vs. $0.3 c(\mathrm{~B})$. Notation for the plots is the same as in the previous Figure. Dynamical ejecta need to be expanding with at least $0.2-0.3 c$ to be responsible for the red excess around 7000-9000 A. Datapoints are as in Figure S2

represents our best models in which we have increased the dynamical ejecta velocity to produce the early red excess in the three points around 7000-8000 $\AA$ (note that velocities here refer to the average speed of the ejecta; the fastest-moving material has a velocity of twice this value). Faster dynamical ejecta ignite the infrared transient earlier, and as a result causes moderate enhancement in the red part of the spectra. We can see that velocity $v_{\text {dyn }}=0.2-0.3 c$ is compatible with observations. Faster wind, however, causes the blue transient to expire sooner and shift the spectrum redwards earlier, which apparently excludes wind velocities as high as $v_{\mathrm{dyn}}=0.16 \mathrm{c}$.

Although the direct contribution of the dynamical ejecta is small, the lanthanides and actinides in its composition can obscure any emission and, for our morphology of the tidal ejecta, the emission in the UV and optical is too obscured for viewing angles more than $40^{\circ}$ off axis. If the viewing angle is greater than this value, the neutron-rich dynamical ejecta must be more constrained to the orbital plane. In another scenario an asymmetric merger may end up with 'kidney-shaped' dynamical ejecta, which only cover a finite sector in azimutal angle (as in (100), their figure 1). If the 'kidney' is facing away from us, it will not obscure the brighter and bluer view of the wind. In either case, it seems that the favored scenario is the one in which the dynamical ejecta do not strongly obscure the wind.

We conclude with the following optimal range of parameters:

- wind composition: first peak elements;

- wind mass: $m_{\text {wind }}=0.03-0.1 M_{\odot}$;

- wind velocity: $v_{\text {wind }}=0.08 c$;

- wind kinetic energy: $E_{\text {wind }}=2 \times 10^{50} \mathrm{erg}$

- dynamical ejecta mass: poorly constrained, compatible with the range from $m_{\mathrm{dyn}}=0.002-0.03 M_{\odot}$;

- dynamical ejecta velocity: $v_{\text {dyn }}=0.2-0.3 c$;

- dynamical ejecta kinetic energy: $E_{\mathrm{dyn}} \sim 6 \times 10^{50} \mathrm{erg}$; 

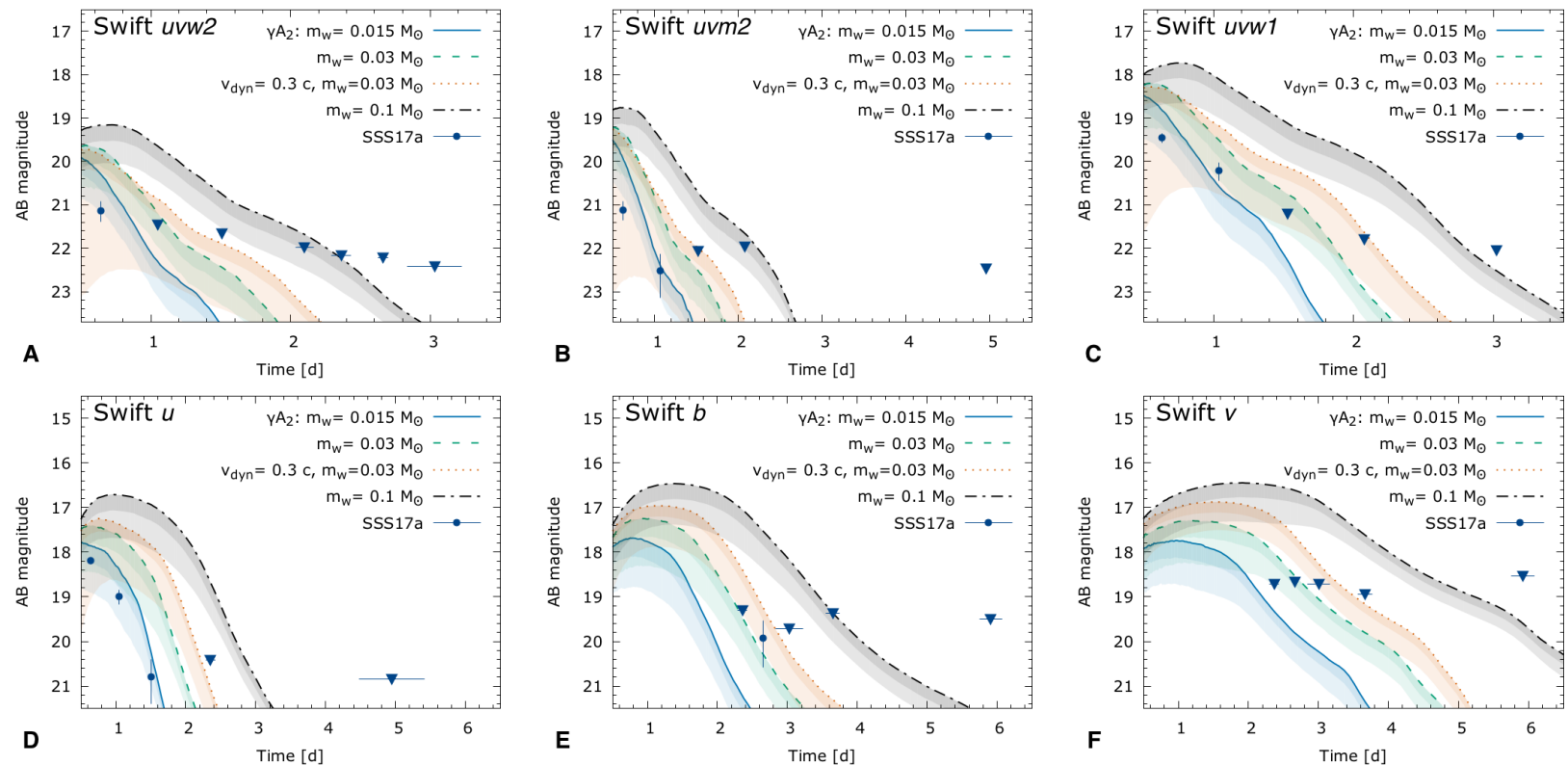

Figure S5: Model light curves for the variants of model configuration $\gamma A_{2}$. The solid lines indicate the predictions for an on-axis viewing geometry, and the shades beaneath are for off-axis geometries, with limiting angles $30^{\circ}$ (darker shade) and $40^{\circ}$ (lighter shade). Datapoints are as in Figure S2

- viewing angle: $<\sim 40^{\circ}$, degenerate with the wind outflow mass: higher polar angle implies higher mass, or non-axisymmetric configuration without dynamical ejecta obscuring the wind.

Figure $\mathrm{S} 5$ presents the light curves in the UV bands for the variations of our baseline model $\gamma A_{2}$.

In addition to the early data from Swift-UVOT, the Very Large Telescope (VLT) in Chile observed EM 170817 with the U filter (similar to the $u$ filter on UVOT) on the Visible wide field Imager and Multi-Object Spectrograph (VIMOS) instrument at $\Delta t=4.47 \mathrm{~d}$. Observations were processed via the esorex software and the photometric zero-point was established using the UVOT data for field stars, transformed to the VLT U-band using the relationsi $\mathrm{n}(68)$. The (extinction corrected) magnitude at this time was $23.35 \pm 0.07(\mathrm{AB})$. Similar results were found by the Hubble Space Telescope (10). These points are not explained by our model, which predicts no emission at these wavelengths at late times (Figure S5D). However, since our focus is on early-time emission certain assumptions were made above (such as neglecting uncertainties on nuclear heating). Thus our which focusses only on the early-time data and reproduced this well, does not give a complete picture of the emission processes at late times.

\subsection{X-ray Emission}

\subsubsection{Afterglow emission}

To place our X-ray limits at the location of EM 170817 in context, we simulated a number of fake sGRB afterglow light curves at a distance of $40 \mathrm{Mpc}$. To do this we took the sample of sGRBs with known redshift from (14), for each of these the best-fitting power-law models from the online XRT GRB Catalog (http://www.swift.ac.uk/xrt_live_cat) (73). Occasionally we manually added extra power-law segments to more accurately reproduce the light curves. This was necessary because for the catalogue extra segments are only added when they are justified at the 4- $\sigma$ level, 
whereas for our purposes we simply want a model that accurately describes the data at the time of our observations. We then scaled the normalisation to correct from the observed redshift to $40 \mathrm{Mpc}$.

In Figure 4 we plot the resultant median light curve and the 25th and 75th percentiles; also shown are the Swift and NUSTAR upper limits. While the sample of sGRBs used is clearly biased, both in sample definition (it is intended to be complete in terms of BAT sensitivity) and our extra selections (objects with no XRT detection cannot be included), this nonetheless indicates that the X-ray afterglow of GRB 170817A is significantly fainter than the majority of previously-observed sGRBs.

This result is not entirely surprising, since the gamma-ray output was orders of magnitude less luminous than the current sample, and this is known to correlate with the X-ray afterglow luminosity. (14) presented correlations between the prompt isotropic energy output and luminosity $\left(E_{\gamma, \text { iso }}, L_{\gamma, \text { iso }}\right)$ and the X-ray flux at 5 hours. Using the Fermi-GBM values of $E_{\gamma \text {,iso }}=4.0 \times 10^{46} \mathrm{erg}$ and $L_{\gamma \text {,iso }}=1.6 \times 10^{47} \mathrm{erg} \mathrm{s}^{-1}$ (25), and assuming a power-law decay of $L \propto t^{-1.5}$ (based on the median light curve in Figure 4), these correlations predict an X-ray luminosity at the time of our first Swift-XRT observation of $4.5 \times 10^{40} \mathrm{erg} \mathrm{s}^{-1}$ (from $E_{\gamma, \text { iso }}$ ) or $2.1 \times 10^{41} \mathrm{erg} \mathrm{s}^{-1}$ (from $L_{\gamma \text {,iso }}$ ). Our upper limit at this time was $2.2 \times 10^{40} \mathrm{erg} \mathrm{s}^{-1}$. Thus, if the X-ray afterglow of GRB 170817A followed the (14) correlations exactly, we would have expected to detect it (though there is some uncertainty in the correlations).

While X-rays were detected by Chandra from EM 170817 at around 9 days after the trigger (no flux was reported for this proprietary observations) $(46-48,76)$, extrapolating the detected X-ray flux back to the times of the Swift and NuSTAR observations using the $t^{-1.5}$ power-law yields a flux above our upper limits (46). Thus our early non-detections also disfavor an on-axis afterglow as the source of the late-time X-ray emission detected by Chandra.

In addition to a comparison with the observed sample of sGRBs, we can translate these limits on X-ray emission from EM 170817 to physical constraints on any associated relativistic ejecta using the standard synchrotron afterglow formulation (103). First, we consider on-axis geometries (i.e. where the relativistic jet is pointed towards Earth).

The X-ray flux will depend on the location of the cooling frequency $\nu_{c}$. At the time of our first Swift-XRT observation $(\Delta t=0.6 \mathrm{~d})$, the cooling frequency will be (104):

$$
\nu_{c}=4 \times 10^{18}\left(\frac{n_{0}}{5 \times 10^{-3} \mathrm{~cm}^{-3}}\right)^{-1}\left(\frac{E_{\mathrm{AG}}}{2 \times 10^{51} \mathrm{erg}}\right)^{-1 / 2} \mathrm{~Hz},
$$

where we have normalized the isotropic afterglow energy $\left(E_{\mathrm{AG}}\right)$ and circumburst density $\left(n_{o}\right)$ to the median values for sGRBs derived in (19). Thus, the XRT bandpass $\left(0.3-10.0 \mathrm{keV}=7.3 \times 10^{16}-2.4 \times 10^{18} \mathrm{~Hz}\right)$ will typically fall near (but slightly below) the cooling frequency at this time, while $\nu_{c}$ will often fall within the (broader) NUSTAR bandpass. (Here and througout, we assume a constant-density circumburst medium, with microphysical parameters: $\epsilon_{e}=0.1$ and $\epsilon_{B}=0.01$, and an electron spectral index of $p=2.5$.)

For $\nu_{X}<\nu_{c}$, our initial NuSTAR upper limit implies (104):

$$
\left(\frac{n_{0}}{5 \times 10^{-3} \mathrm{~cm}^{-3}}\right)^{1 / 2}\left(\frac{E_{\mathrm{AG}}}{2 \times 10^{51} \mathrm{erg}}\right)^{11 / 8}<2 \times 10^{-4} .
$$

As a result, for any reasonable circumburst density $\left(n_{0}>\sim 10^{-5} \mathrm{~cm}^{-3} ;(19)\right)$, we place a limit on the amount of energy coupled to relativistic ejecta along our line of sight of $E_{\mathrm{AG}}<\sim 10^{50} \mathrm{erg}$. Adopting $\nu_{X}>\nu_{c}$ yields similar constraints (though the result is independent of $n_{0}$ ). Utilizing the first Swift-XRT limit results in a comparable limit on the energy coupled to relativistic ejecta (for on-axis geometries). 
To verify these results, we ran a series of simulations using the afterglow light curve code BOXFIT (23). Over the observed range of circumburst densities and afterglow energies for sGRBs (19), we calculated the predicted $\mathrm{X}$-ray flux at the time of our initial NuSTAR observation $(\Delta t=0.7 \mathrm{~d})$. The results are shown in the bottom panel of Figure 4. Similar to our analytic results above, we can rule out the presence of a collimated, relativistic outflow initially beamed towards Earth with $E_{\mathrm{AG}}>\sim 10^{50} \mathrm{erg}$.

As discussed by (10), the prompt low $\gamma$-ray luminosity of GRB 170817A is not consistent with emission from a standard ultra-relativistic jet as seen in sGRBs to date, regardless of viewing angle. Instead it must arise from some previously unseen process, such as a heated cocoon (e.g. $(26,27)$ ); and we are likely viewing the event off-axis (unless there was no jet at all), as indeed our modelling of the UV emission (above) suggests. In this case, if there is an ultrarelativistic jet which is oriented away from us, we can expect X-ray emission at late times. As the jet decelerates and spreads laterally, it illuminates an increasing fraction of the sky, thus eventually spreading into our line of sight. Such "orphan" afterglow emission is expected to be much fainter than the early afterglow, but due to the low distance to GW 170817 may be detectable by Swift.

We therefore repeated the afterglow light curve simulations described above for a variety of off-axis viewing geometries. For the median values of short-duration GRB afterglow energy $\left(E_{\mathrm{AG}}=2 \times 10^{51} \mathrm{erg}\right)$ and circumburst density $\left(n_{0}=5 \times 10^{-3} \mathrm{~cm}^{-3} ;(19)\right)$, the resulting light curves are plotted in Figure 5. With the XRT and NuSTAR non-detections to date, we can rule out any viewing angle with $\theta_{\text {obs }}<\sim 20^{\circ}$. With these afterglow parameters, the reported Chandra flux of $5 \times 10^{-15} \mathrm{erg} \mathrm{cm}^{-2} \mathrm{~s}^{-1}(105,106)$ can be reproduced with a viewing angle of $\theta_{\mathrm{obs}} \approx 30^{\circ}$. These constraints are consistent with the geometry implied by our modelling of the blue kilonova seen by the UVOT. It is also broadly consistent with the evolution of EM 170817 at radio wavelengths (9), showing no emission at early times but then later detections $(107,108)$.

\subsubsection{Emission from a heated cocoon}

One way of explaining the observed prompt gamma-rays may be via the 'cocoon model' (26,27). In this model, if the GRB jet propagates through a baryon-contaminated site around the merger, a heated cocoon is formed from which prompt gamma-rays may be seen. Under the (27) model, the prompt luminosity of GRB 170817A can be interpreted as arising from a typical (or rather, Swift-like) sGRB viewed $\sim 10-20^{\circ}$ off-axis. In their revised model, in which the cocoon is not isotropic (109), the prompt gamma-ray luminosity implies an observing angle of $\sim 40$ $50^{\circ}$ compared to the jet angle. However, the peak photon energy reported by $(81)(124.2 \pm 52.6 \mathrm{keV})$ requires a viewing angle of $\sim 10^{\circ}$ according to the same model, which suggests that this model, in its current form at least, cannot explain the observed prompt emission.

Nonetheless, a heated cocoon may in principle give rise to late-time X-ray emission, as the 'trans-relativistic' expanding cocoon (i.e. with Lorentz factor $\Gamma \sim 2-3$ ) is eventually decelerated by the ambient medium which then shocks and radiates in a manner analagous to the standard on-axis GRB afterglow (26). In the main text we demonstrated that our upper limits and the Chandra detections are consistent with most of these models, which predict a peak X-ray flux several weeks to months after the merger.

\subsubsection{Non-afterglow emission}

In addition to the emission associated directly or indirectly with the jet, as above, some authors have predicted quasi-isotropic X-ray emission following a binary neutron star merger. (110), responding to an apparent X-ray excess in the light curve of GRB 130603B (111) coincident with a claimed kilonova detection $(53,112)$, proposed that the kilonova was powered not by $r$-process nucleosynthesis but by isotropic X-ray emission, perhaps from 
fallback accretion via a disk. They predict typical X-ray luminosities of order $10^{41} \mathrm{erg} \mathrm{s}^{-1}$ appearing at around 7 days after the merger. We find no evidence for such emission in our data, with upper limits of $\sim 1.5 \times 10^{40} \mathrm{erg}$ $\mathrm{s}^{-1}$ at 7 days after the trigger.

Chandra detected X-ray emission from EM 170817 at $\sim 9 \mathrm{~d}$ after the trigger (46) and in subsequent observations $(47,48,76,105,106,113)$ with a luminosity of $\sim 9 \times 10^{38} \mathrm{erg} \mathrm{s}^{-1}(0.3-8 \mathrm{keV})$, consistent with our XRT and NUSTAR upper limits. This could be indicative of the emission predicted by (110), if the accretion rate is much lower than in their fiducial calculations. Alternatively, those authors note that if the ejecta initially prevent the detection of the X-rays (i.e. the X-rays are all thermalized by the optically thick ejecta) then the ejecta will still become optically thin to X-rays at tens of days, with luminosities $\sim 10^{40}-10^{41} \mathrm{erg} \mathrm{s}^{-1}$, still well above both the $\mathrm{XRT} /$ NUSTAR detection thresholds and the detected Chandra flux, thus testable with future observations.

It has also been considered that the result of the BNS merger may be a hyper-massive neutron-star which is able to support itself at least temporarily against gravitational collapse, e.g. by rotation, and which emits X-rays as it rotates. For example $(22,114-116)$ succesfully modelled early-time X-ray light curve plateaux as being powered by the spin-down of a magnetar. However such plateaux do not extend to the times at which our observations of EM 170817 began, and in some cases imply the collapse of the magnetar within a few ks of the merger, well before our X-ray observations of EM 170817 began.

(117) proposed three mechanisms for producing X-rays after a binary neutron star merger: the standard afterglow emission, spin-down energy extracted from a magnetar, or a 'merger-nova' in which the kilonova-emitting ejecta are heated also by the magnetar and emit X-rays (this latter case was also proposed by (118) in whose model this emission is extremely bright, however (117) attribute this to an error and predict dramatically less X-ray flux). In this model, the observed X-ray emission depends upon the viewing angle; with the observer either looking down the GRB jet, missing the jet but having an unobstructed view of the magnetar, or missing the jet, and viewing the magnetar through the ejecta. Based on these assumptions, (117) produced a set of example light curves for typical sGRB parameters. Almost all of their light curves predict luminosities 4-5 orders of magnitude above the X-ray detection limits presented here, thus even if the available energy in the magnetar is substatially reduced compared to typical, we would have detected this emission.

However, the X-ray flux is greatly reduced in the case that the magnetar collapses to a black hole before our observations (consistent with the plateau durations of $(22,114-116)$ ), or the collapse timescale of the magnetar is shorter than its spindown timescale. In light of these considerations, the lack of X-ray emission above $\sim 1.5 \times 10^{40}$ $\mathrm{erg} \mathrm{s}^{-1}$ strongly suggests that if a magnetar was formed after the BNS merger, it collapsed to a black hole within the first $0.6 \mathrm{~d}$ of its formation.

\subsubsection{Limits on nuclear line emission}

Radioactive $r$-process nucleii synthesized in the merger ejecta produce gamma-ray lines through $\beta$ decay. The emission lines have a broad spectrum from $10 \mathrm{keV}$ to $1 \mathrm{MeV}(98,119))$. These lines start to emerge from the ejecta when the ejecta become optically thin. We model the flux of the nuclear emission lines for the ejecta mass of $0.03 M_{\odot}$ and $0.05 M_{\odot}$ with the mean expansion velocity of $0.2 c(119)$, taking photoelectric absorption and the Compton scattering into account. Here we assume a composition of nuclei that matches the solar $r$-process abundance pattern of stable and long-lived nuclei for $90 \leq A \leq 140$, where $A$ is the atomic mass number. There are three strong lines around 28,50 , and $81 \mathrm{keV}$, which are produced by ${ }^{129} \mathrm{Te},{ }^{132} \mathrm{Te}$, and ${ }^{133} \mathrm{Xe}$. However, the line nuclear emission is too weak to be detected by NUSTAR, as shown in Figure 66. 


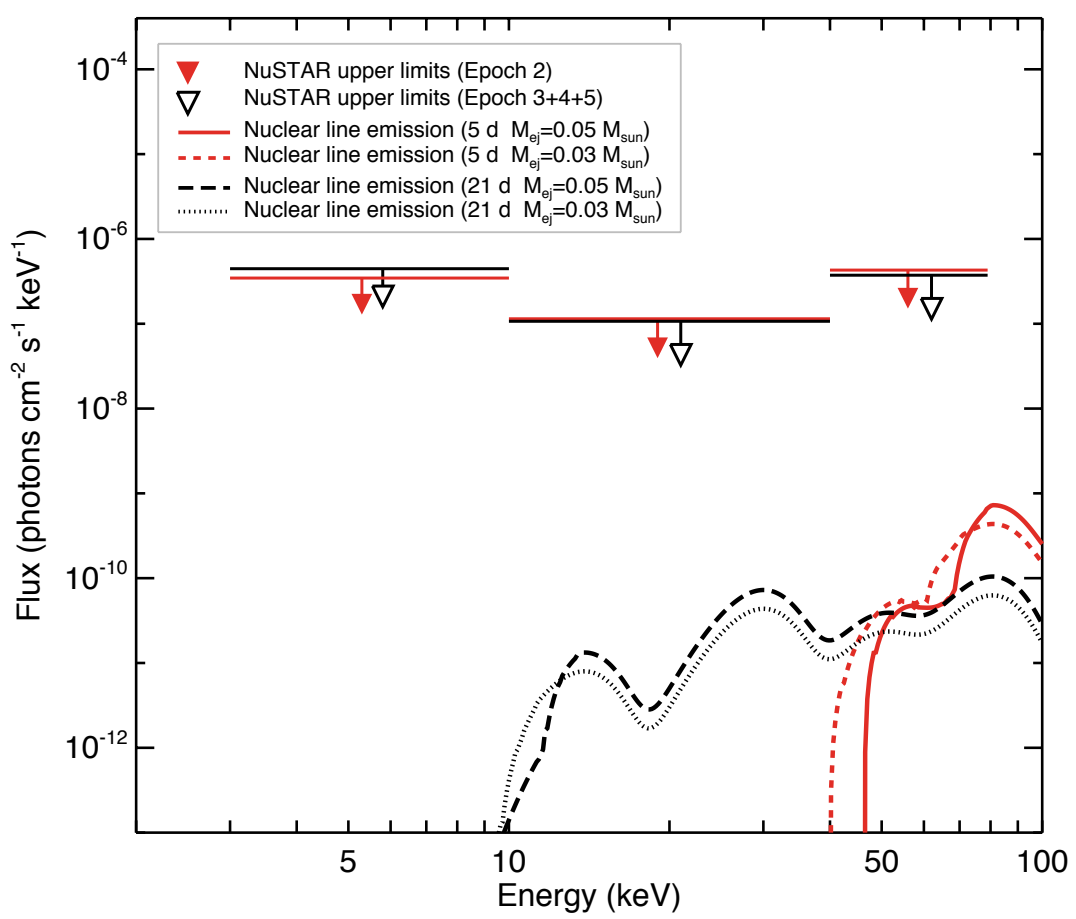

Figure S6: Predicted X-ray flux from atomic line emission of high atomic mass elements. The predicted flux falls well below the sensitivity of our NUSTAR data. 


\subsection{Constraints on X-ray emission from the wide-angle search.}

Our discussion so far has assumed that EM 170817, GRB 170817A and GW 170817 are all the same event. While this seems reasonable on probabilistic grounds $($ e.g. $(8,120))$ it is not certain. For this reason, even after the detection of EM 170817, Swift continued to search the GW error region for possible counterparts. In total the tiling observations continued until $4.3 \mathrm{~d}$ after the GW event, and XRT covered $52 \%$ of the probability in the three-detector 'prelimary-LALInference' skymap (13), and $92 \%$ of the probability determined by convolving this skymap with GWGC, as described in (12). Therefore, if EM 170817 is not the counterpart to GW 170817 it is probable that Swift-XRT observed the true location of the event.

In Figure 4 we show a sample of sGRB X-ray light curves as they would have appeared at $40 \mathrm{Mpc}$ (the bestfitting luminosity distance in the 3D GW skymap). The blue line on this plot shows the time-range spanned by our search of the GW error region and the detection limit of the observations. This shows that a sGRB afterglow typical of those observed to date would have been detectable with XRT during our follow up. As noted above, some known sGRBs have extremely fast-fading afterglows (22); such objects fall below the 25th percentile of GRB flux by the times of our observations so cannot be seen in Figure 4 and would not have been detected in our search.

To investigate in more detail, we simulated 10,000 sGRBs. Their position and distance were drawn at random from the 3-D 'bayestar-HLV' skymap (11) (i.e. that on which our tiles were based), and their light curves were produced by taking a random sGRB from the sample described in Section 3.3.1 and scaling it to the distance of the simulated event. We then identified for each of these simulated events whether its location was observed by XRT, and if so, whether the source would have been above our detection limit at the time of the observation. We found that in $65 \%$ of cases the source would have been detected. We then repeated the process, but instead of using the actual locations observed by XRT, which included many observations of EM 170817 which displaced planned tiling of the GW-GRB probability region, we used those which would have been observed if we had not focussed on EM 170817. In these simulations 68\% of the simulated sources were recovered. Since GRB 170817A was much fainter than typical Swift-detected sGRBs, we repeated these simulations again, this time reducing the predicted XRT flux by a factor of 1,000 . In this case we find we would have detected $11 \%$ of simulated events, or $10 \%$ if we had not targeted EM 170817. The slight decrease when not targeting EM 170817 - i.e. when covering more of the GW error region, arises because the focus on EM 170817 results in longer exposures and hence the ability to detect fainter sources in that field, which in this case outweighs the benefits of searching more fields at a lower sensitivity. 


\section{References}

1. The LIGO Scientific Collaboration, the Virgo Collaboration, Gamma Ray Coordinates Network Circular 21505 (2017).

2. The LIGO Scientific Collaboration, the Virgo Collaboration, Phys. Rev. Lett: DOI to be supplied ASAP (2017).

3. B. P. Abbott, et al., Physical Review X 6, 041015 (2016).

4. V. Connaughton, et al., Gamma Ray Coordinates Network Circular 21506 (2017).

5. A. Goldstein, et al., ApJL, submitted .

6. N. Gehrels, et al., Astrophys. J 611, 1005 (2004).

7. Materials and methods are available as supplementary materials on Science Online .

8. The LIGO Scientific Collaboration, the Virgo Collaboration, Phys. Rev. Lett: DOI to be supplied ASAP (2017).

9. G. Hallinan, et al., Science - DOI: 10.1126/science.aap9855 (2017).

10. M. M. Kasliwal, et al., Science - DOI: 10.1126/science.aap9455 (2017).

11. The LIGO Scientific Collaboration, the Virgo Collaboration, Gamma Ray Coordinates Network Circular 21513 (2017).

12. P. A. Evans, et al., Mon. Not. R. Astron. Soc 462, 1591 (2016).

13. The LIGO Scientific Collaboration, the Virgo Collaboration, Gamma Ray Coordinates Network Circular 21527 (2017).

14. P. D’Avanzo, et al., Mon. Not. R. Astron. Soc 442, 2342 (2014).

15. D. Coulter, et al., Gamma Ray Coordinates Network Circular 21529 (2017).

16. D. Coulter, et al., Science, doi:10.1126/science.aap9811.

17. F. A. Harrison, et al., Astrophys. J 770, 103 (2013).

18. S. Smartt, et al., Nature, DOI: 10.1038/nature24303.

19. W. Fong, E. Berger, R. Margutti, B. A. Zauderer, Astrophys. J 815, 102 (2015).

20. P. Meszaros, M. J. Rees, Astrophys. J. 405, 278 (1993).

21. T. Piran, Reviews of Modern Physics 76, 1143 (2004).

22. A. Rowlinson, et al., Mon. Not. R. Astron. Soc 409, 531 (2010).

23. H. van Eerten, A. van der Horst, A. MacFadyen, Astrophys. J 749, 44 (2012). 
Table S3: Swift observations of the error region of GW 170817A.

\begin{tabular}{|c|c|c|}
\hline $\begin{array}{l}\text { Pointing direction } \\
\quad \text { (J2000) }\end{array}$ & $\begin{array}{l}\text { Start time }{ }^{a} \\
\text { (UTC) }\end{array}$ & $\begin{array}{l}\text { Exposure } \\
\text { (s) }\end{array}$ \\
\hline $11^{h} 47^{m} 9.20^{s},-39 \operatorname{deg} 50^{\prime} 40.2^{\prime \prime}$ & Aug 17 at 13:37:07 (0.039) & 117 \\
\hline $11^{h} 48^{m} 57.01^{s},-39 \operatorname{deg} 50^{\prime} 27.9^{\prime \prime}$ & Aug 17 at 13:38:13 (0.040) & 80 \\
\hline $11^{h} 48^{m} 1.57^{s},-40 \operatorname{deg} 08^{\prime} 14.1^{\prime \prime}$ & Aug 17 at 13:39:19 (0.040) & 85 \\
\hline $11^{h} 46^{m} 15.97^{s},-40 \operatorname{deg} 08^{\prime} 01.1^{\prime \prime}$ & Aug 17 at 13:40:24 (0.041) & 90 \\
\hline $11^{h} 45^{m} 23.31^{s},-39 \operatorname{deg} 50^{\prime} 03.0^{\prime \prime}$ & Aug 17 at 13:41:30 (0.042) & 125 \\
\hline $11^{h} 46^{m} 18.37^{s},-39 \operatorname{deg} 32^{\prime} 28.2^{\prime \prime}$ & Aug 17 at 13:42:35 (0.043) & 87 \\
\hline $11^{h} 48^{m} 4.49^{s},-39 \operatorname{deg} 32^{\prime} 53.9^{\prime \prime}$ & Aug 17 at 13:43:41 (0.043) & 122 \\
\hline $11^{h} 49^{m} 49.51^{s},-39 \operatorname{deg} 32^{\prime} 55.3^{\prime \prime}$ & Aug 17 at 13:44:47 (0.044) & 82 \\
\hline $11^{h} 50^{m} 41.69^{s},-39 \operatorname{deg} 50^{\prime} 40.1^{\prime \prime}$ & Aug 17 at $13: 45: 52(0.045)$ & 85 \\
\hline $11^{h} 49^{m} 47.40^{s},-40 \operatorname{deg} 08^{\prime} 18.8^{\prime \prime}$ & Aug 17 at 13:46:58 (0.046) & 122 \\
\hline $11^{h} 48^{m} 53.74^{s},-40 \operatorname{deg} 25^{\prime} 48.7^{\prime \prime}$ & Aug 17 at 13:48:03 (0.047) & 87 \\
\hline $11^{h} 47^{m} 8.17^{s},-40 \operatorname{deg} 25^{\prime} 28.4^{\prime \prime}$ & Aug 17 at 13:49:09 (0.047) & 82 \\
\hline $11^{h} 45^{m} 22.57^{s},-40 \operatorname{deg} 25^{\prime} 31.7^{\prime \prime}$ & Aug 17 at 13:50:15 (0.048) & 135 \\
\hline $11^{h} 44^{m} 31.01^{s},-40 \operatorname{deg} 07^{\prime} 37.1^{\prime \prime}$ & Aug 17 at 14:47:11 (0.088) & 85 \\
\hline $11^{h} 43^{m} 39.46^{s},-39 \operatorname{deg} 49^{\prime} 52.3^{\prime \prime}$ & Aug 17 at 14:48:23(0.088) & 92 \\
\hline $11^{h} 44^{m} 32.85^{s},-39 \operatorname{deg} 32^{\prime} 32.2^{\prime \prime}$ & Aug 17 at 14:49:34 (0.089) & 90 \\
\hline $11^{h} 45^{m} 25.60^{s},-39 \operatorname{deg} 14^{\prime} 57.5^{\prime \prime}$ & Aug 17 at 14:50:44 (0.090) & 85 \\
\hline $11^{h} 47^{m} 12.13^{s},-39 \operatorname{deg} 15^{\prime} 21.6^{\prime \prime}$ & Aug 17 at 14:51:55 (0.091) & 90 \\
\hline $11^{h} 48^{m} 56.02^{s},-39 \operatorname{deg} 15^{\prime} 25.5^{\prime \prime}$ & Aug 17 at 14:53:06 (0.092) & 90 \\
\hline $11^{h} 50^{m} 41.63^{s},-39 \operatorname{deg} 15^{\prime} 19.1^{\prime \prime}$ & Aug 17 at 14:54:17 (0.093) & 85 \\
\hline $11^{h} 51^{m} 34.27^{s},-39 \operatorname{deg} 33^{\prime} 09.8^{\prime \prime}$ & Aug 17 at 14:55:27 (0.093) & 87 \\
\hline $11^{h} 52^{m} 26.69^{s},-39 \operatorname{deg} 50^{\prime} 48.2^{\prime \prime}$ & Aug 17 at 14:56:38 (0.094) & 90 \\
\hline $11^{h} 51^{m} 31.09^{s},-40 \operatorname{deg} 08^{\prime} 17.0^{\prime \prime}$ & Aug 17 at 14:57:49 (0.095) & 90 \\
\hline $11^{h} 50^{m} 39.51^{s},-40 \operatorname{deg} 25^{\prime} 39.4^{\prime \prime}$ & Aug 17 at 14:59:00 (0.096) & 90 \\
\hline $11^{h} 49^{m} 46.61^{s},-40 \operatorname{deg} 43^{\prime} 10.6^{\prime \prime}$ & Aug 17 at 15:00:10 (0.097) & 87 \\
\hline $11^{h} 48^{m} 0.06^{s},-40 \operatorname{deg} 42^{\prime} 48.2^{\prime \prime}$ & Aug 17 at 15:01:21 (0.097) & 87 \\
\hline $11^{h} 46^{m} 15.82^{s},-40 \operatorname{deg} 42^{\prime} 53.4^{\prime \prime}$ & Aug 17 at 15:02:31 (0.098) & 87 \\
\hline $11^{h} 44^{m} 29.36^{s},-40 \operatorname{deg} 42^{\prime} 49.7^{\prime \prime}$ & Aug 17 at 15:03:41 (0.099) & 87 \\
\hline $11^{h} 43^{m} 38.27^{s},-40 \operatorname{deg} 25^{\prime} 10.7^{\prime \prime}$ & Aug 17 at 15:04:49 (0.100) & 85 \\
\hline $11^{h} 42^{m} 45.53^{s},-40 \operatorname{deg} 07^{\prime} 26.9^{\prime \prime}$ & Aug 17 at 15:05:57 (0.10) & 87 \\
\hline $11^{h} 41^{m} 53.04^{s},-39 \operatorname{deg} 49^{\prime} 54.0^{\prime \prime}$ & Aug 17 at 15:07:05 (0.10) & 85 \\
\hline $11^{h} 42^{m} 47.85^{s},-39 \operatorname{deg} 32^{\prime} 33.8^{\prime \prime}$ & Aug 17 at 15:08:12 (0.10) & 80 \\
\hline $11^{h} 43^{m} 39.73^{s},-39 \operatorname{deg} 15^{\prime} 04.2^{\prime \prime}$ & Aug 17 at 15:09:17 (0.10) & 85 \\
\hline $11^{h} 44^{m} 33.29^{s},-38 \operatorname{deg} 57^{\prime} 26.2^{\prime \prime}$ & Aug 17 at $15: 10: 23(0.10)$ & 87 \\
\hline $11^{h} 46^{m} 18.76^{s},-38 \operatorname{deg} 57^{\prime} 49.4^{\prime \prime}$ & Aug 17 at 15:11:29 (0.10) & 77 \\
\hline
\end{tabular}

${ }^{a}$ Observations were 2017, times in parentheses are days since the GW trigger. 


\begin{tabular}{|c|c|c|}
\hline $\begin{array}{l}\text { Pointing direction } \\
(\mathrm{J} 2000)\end{array}$ & $\begin{array}{l}\text { Start time }^{a} \\
\text { (UTC) }\end{array}$ & $\begin{array}{c}\text { Exposure } \\
\text { (s) }\end{array}$ \\
\hline $11^{h} 48^{m} 3.97^{s},-38 \operatorname{deg} 57^{\prime} 51.7^{\prime \prime}$ & Aug 17 at $15: 12: 34(0.11)$ & 85 \\
\hline $11^{h} 49^{m} 49.45^{s},-38 \operatorname{deg} 57^{\prime} 44.9^{\prime \prime}$ & Aug 17 at $15: 13: 40(0.11)$ & 90 \\
\hline $15^{h} 02^{m} 24.24^{s},-43 \operatorname{deg} 31^{\prime} 11.6^{\prime \prime}$ & Aug 17 at $17: 16: 33(0.19)$ & 15 \\
\hline $16^{h} 14^{m} 12.96^{s},-61 \operatorname{deg} 03^{\prime} 11.2^{\prime \prime}$ & Aug 17 at $17: 17: 28(0.19)$ & 57 \\
\hline $09^{h} 16^{m} 10.08^{s},-37 \operatorname{deg} 53^{\prime} 03.5^{\prime \prime}$ & Aug 17 at $17: 50: 11(0.21)$ & 27 \\
\hline $10^{h} 30^{m} 24.24^{s},-35 \operatorname{deg} 03^{\prime} 59.0^{\prime \prime}$ & Aug 17 at 17:51:07 (0.22) & 52 \\
\hline $10^{h} 40^{m} 22.80^{s},-36 \operatorname{deg} 19^{\prime} 07.7^{\prime \prime}$ & Aug 17 at $17: 52: 57(0.22)$ & 60 \\
\hline $10^{h} 41^{m} 56.16^{s},-36 \operatorname{deg} 19^{\prime} 07.7^{\prime \prime}$ & Aug 17 at $17: 54: 29(0.22)$ & 60 \\
\hline $11^{h} 08^{m} 49.68^{s},-37 \operatorname{deg} 53^{\prime} 03.5^{\prime \prime}$ & Aug 17 at $17: 55: 54(0.22)$ & 52 \\
\hline $11^{h} 06^{m} 22.56^{s},-37 \operatorname{deg} 15^{\prime} 29.2^{\prime \prime}$ & Aug 17 at $17: 57: 31(0.22)$ & 62 \\
\hline $11^{h} 37^{m} 23.52^{s},-37 \operatorname{deg} 53^{\prime} 03.5^{\prime \prime}$ & Aug 17 at $17: 59: 03(0.22)$ & 55 \\
\hline $11^{h} 51^{m} 24.96^{s},-38 \operatorname{deg} 30^{\prime} 37.4^{\prime \prime}$ & Aug 17 at $18: 00: 41(0.22)$ & 60 \\
\hline $11^{h} 52^{m} 7.44^{s},-38 \operatorname{deg} 49^{\prime} 24.6^{\prime \prime}$ & Aug 17 at $18: 02: 15(0.22)$ & 57 \\
\hline $11^{h} 53^{m} 37.20^{s},-39 \operatorname{deg} 26^{\prime} 58.9^{\prime \prime}$ & Aug 17 at $18: 03: 40(0.22)$ & 62 \\
\hline $11^{h} 55^{m} 14.40^{s},-39 \operatorname{deg} 26^{\prime} 58.9^{\prime \prime}$ & Aug 17 at $18: 05: 11(0.23)$ & 35 \\
\hline $12^{h} 00^{m} 6.24^{s},-39 \operatorname{deg} 26^{\prime} 58.9^{\prime \prime}$ & Aug 17 at $18: 06: 36(0.23)$ & 55 \\
\hline $12^{h} 50^{m} 58.32^{s},-41 \operatorname{deg} 00^{\prime} 54.7^{\prime \prime}$ & Aug 17 at 18:08:07 (0.23) & 57 \\
\hline $12^{h} 53^{m} 23.52^{s},-41 \operatorname{deg} 38^{\prime} 29.0^{\prime \prime}$ & Aug 17 at 18:09:50 (0.23) & 62 \\
\hline $12^{h} 52^{m} 58.08^{s},-41 \operatorname{deg} 57^{\prime} 16.2^{\prime \prime}$ & Aug 17 at $18: 11: 21(0.23)$ & 55 \\
\hline $12^{h} 48^{m} 21.84^{s},-41 \operatorname{deg} 38^{\prime} 29.0^{\prime \prime}$ & Aug 17 at $18: 12: 46(0.23)$ & 62 \\
\hline $12^{h} 47^{m} 54.96^{s},-41 \operatorname{deg} 57^{\prime} 16.2^{\prime \prime}$ & Aug 17 at $18: 14: 16(0.23)$ & 60 \\
\hline $12^{h} 45^{m} 59.52^{s},-41 \operatorname{deg} 00^{\prime} 54.7^{\prime \prime}$ & Aug 17 at $18: 15: 41(0.23)$ & 57 \\
\hline $12^{h} 44^{m} 19.92^{s},-41 \operatorname{deg} 00^{\prime} 54.7^{\prime \prime}$ & Aug 17 at $18: 17: 13(0.23)$ & 55 \\
\hline $12^{h} 39^{m} 21.36^{s},-41 \operatorname{deg} 00^{\prime} 54.7^{\prime \prime}$ & Aug 17 at $18: 18: 38(0.23)$ & 62 \\
\hline $12^{h} 37^{m} 10.80^{s},-40 \operatorname{deg} 23^{\prime} 20.4^{\prime \prime}$ & Aug 17 at 18:20:08 (0.24) & 62 \\
\hline $12^{h} 36^{m} 8.64^{s},-40 \operatorname{deg} 04^{\prime} 33.2^{\prime \prime}$ & Aug 17 at $18: 21: 40(0.24)$ & 67 \\
\hline $12^{h} 25^{m} 40.32^{s},-40 \operatorname{deg} 23^{\prime} 20.4^{\prime \prime}$ & Aug 17 at $18: 23: 11(0.24)$ & 60 \\
\hline $12^{h} 24^{m} 41.28^{s},-40 \operatorname{deg} 04^{\prime} 33.2^{\prime \prime}$ & Aug 17 at $18: 24: 43(0.24)$ & 55 \\
\hline $12^{h} 25^{m} 52.80^{s},-38 \operatorname{deg} 49^{\prime} 24.6^{\prime \prime}$ & Aug 17 at 18:26:08 (0.24) & 60 \\
\hline $12^{h} 25^{m} 1.68^{s},-38 \operatorname{deg} 30^{\prime} 37.4^{\prime \prime}$ & Aug 17 at $18: 27: 40(0.24)$ & 60 \\
\hline $12^{h} 47^{m} 36.00^{s},-40 \operatorname{deg} 04^{\prime} 33.2^{\prime \prime}$ & Aug 17 at $18: 29: 05(0.24)$ & 57 \\
\hline $12^{h} 42^{m} 41.52^{s},-40 \operatorname{deg} 04^{\prime} 33.2^{\prime \prime}$ & Aug 17 at $18: 30: 41(0.24)$ & 60 \\
\hline $12^{h} 42^{m} 6.96^{s},-40 \operatorname{deg} 23^{\prime} 20.4^{\prime \prime}$ & Aug 17 at $18: 32: 12(0.24)$ & 60 \\
\hline $12^{h} 53^{m} 37.44^{s},-40 \operatorname{deg} 23^{\prime} 20.4^{\prime \prime}$ & Aug 17 at $18: 33: 37(0.24)$ & 60 \\
\hline $12^{h} 54^{m} 8.88^{s},-40 \operatorname{deg} 04^{\prime} 33.2^{\prime \prime}$ & Aug 17 at 18:35:09 (0.25) & 57 \\
\hline $12^{h} 53^{m} 12.48^{s},-38 \operatorname{deg} 49^{\prime} 24.6^{\prime \prime}$ & Aug 17 at $18: 36: 35(0.25)$ & 62 \\
\hline
\end{tabular}

${ }^{a}$ Observations were 2017, times in parentheses are days since the GW trigger. 


\begin{tabular}{|c|c|c|}
\hline $\begin{array}{l}\text { Pointing direction } \\
\text { (J2000) }\end{array}$ & $\begin{array}{l}\text { Start time }^{a} \\
\text { (UTC) }\end{array}$ & $\begin{array}{c}\text { Exposure } \\
\text { (s) }\end{array}$ \\
\hline $12^{h} 53^{m} 50.16^{s},-38 \operatorname{deg} 30^{\prime} 37.4^{\prime \prime}$ & Aug 17 at $18: 38: 07(0.25)$ & 57 \\
\hline $12^{h} 55^{m} 14.88^{s},-39 \operatorname{deg} 26^{\prime} 58.9^{\prime \prime}$ & Aug 17 at $18: 39: 32(0.25)$ & 62 \\
\hline $12^{h} 56^{m} 52.32^{s},-39 \operatorname{deg} 26^{\prime} 58.9^{\prime \prime}$ & Aug 17 at $18: 41: 03(0.25)$ & 60 \\
\hline $13^{h} 11^{m} 52.08^{s},-43 \operatorname{deg} 31^{\prime} 11.6^{\prime \prime}$ & Aug 17 at $18: 42: 27(0.25)$ & 57 \\
\hline $13^{h} 20^{m} 13.44^{s},-44 \operatorname{deg} 08^{\prime} 46.0^{\prime \prime}$ & Aug 17 at $18: 44: 05(0.25)$ & 60 \\
\hline $14^{h} 03^{m} 18.00^{s},-33 \operatorname{deg} 30^{\prime} 03.2^{\prime \prime}$ & Aug 17 at $18: 45: 37(0.25)$ & 57 \\
\hline $14^{h} 17^{m} 4.08^{s},-48 \operatorname{deg} 12^{\prime} 58.7^{\prime \prime}$ & Aug 17 at $18: 47: 24(0.25)$ & 57 \\
\hline $14^{h} 18^{m} 56.88^{s},-48 \operatorname{deg} 12^{\prime} 58.7^{\prime \prime}$ & Aug 17 at $18: 49: 14(0.26)$ & 57 \\
\hline $14^{h} 57^{m} 35.28^{s},-41 \operatorname{deg} 57^{\prime} 16.2^{\prime \prime}$ & Aug 17 at $18: 50: 39(0.26)$ & 55 \\
\hline $14^{h} 56^{m} 41.28^{s},-54 \operatorname{deg} 28^{\prime} 41.5^{\prime \prime}$ & Aug 17 at $18: 52: 21(0.26)$ & 55 \\
\hline $10^{h} 21^{m} 2.88^{s},-34 \operatorname{deg} 07^{\prime} 37.6^{\prime \prime}$ & Aug 17 at $19: 26: 30(0.28)$ & 40 \\
\hline $10^{h} 27^{m} 26.16^{s},-44 \operatorname{deg} 08^{\prime} 46.0^{\prime \prime}$ & Aug 17 at $19: 28: 00(0.28)$ & 47 \\
\hline $10^{h} 29^{m} 11.04^{s},-44 \operatorname{deg} 08^{\prime} 46.0^{\prime \prime}$ & Aug 17 at $19: 29: 42(0.28)$ & 50 \\
\hline $10^{h} 27^{m} 17.04^{s},-40 \operatorname{deg} 23^{\prime} 20.4^{\prime \prime}$ & Aug 17 at $19: 31: 08(0.28)$ & 57 \\
\hline $10^{h} 26^{m} 50.88^{s},-40 \operatorname{deg} 04^{\prime} 33.2^{\prime \prime}$ & Aug 17 at $19: 32: 43(0.29)$ & 60 \\
\hline $11^{h} 31^{m} 40.56^{s},-36 \operatorname{deg} 19^{\prime} 07.7^{\prime \prime}$ & Aug 17 at 19:34:08 (0.29) & 57 \\
\hline $11^{h} 54^{m} 50.64^{s},-37 \operatorname{deg} 53^{\prime} 03.5^{\prime \prime}$ & Aug 17 at $19: 35: 55(0.29)$ & 57 \\
\hline $12^{h} 14^{m} 0.24^{s},-35 \operatorname{deg} 41^{\prime} 33.4^{\prime \prime}$ & Aug 17 at $19: 37: 31(0.29)$ & 5 \\
\hline $12^{h} 22^{m} 6.00^{s},-35 \operatorname{deg} 03^{\prime} 59.0^{\prime \prime}$ & Aug 17 at 19:39:08 (0.29) & 60 \\
\hline $12^{h} 20^{m} 10.32^{s},-35 \operatorname{deg} 41^{\prime} 33.4^{\prime \prime}$ & Aug 17 at $19: 40: 40(0.29)$ & 40 \\
\hline $12^{h} 17^{m} 3.60^{s},-37 \operatorname{deg} 53^{\prime} 03.5^{\prime \prime}$ & Aug 17 at $19: 42: 10(0.29)$ & 62 \\
\hline $12^{h} 20^{m} 13.44^{s},-38 \operatorname{deg} 30^{\prime} 37.4^{\prime \prime}$ & Aug 17 at $19: 43: 44(0.29)$ & 62 \\
\hline $12^{h} 19^{m} 26.88^{s},-38 \operatorname{deg} 49^{\prime} 24.6^{\prime \prime}$ & Aug 17 at $19: 45: 15(0.29)$ & 57 \\
\hline $12^{h} 08^{m} 19.20^{s},-40 \operatorname{deg} 04^{\prime} 33.2^{\prime \prime}$ & Aug 17 at $19: 46: 40(0.30)$ & 60 \\
\hline $12^{h} 06^{m} 35.52^{s},-39 \operatorname{deg} 26^{\prime} 58.9^{\prime \prime}$ & Aug 17 at $19: 48: 13(0.30)$ & 57 \\
\hline $12^{h} 04^{m} 58.32^{s},-39 \operatorname{deg} 26^{\prime} 58.9^{\prime \prime}$ & Aug 17 at $19: 49: 44(0.30)$ & 60 \\
\hline $13^{h} 04^{m} 14.64^{s},-30 \operatorname{deg} 03^{\prime} 24.8^{\prime \prime}$ & Aug 17 at $19: 51: 09(0.30)$ & 55 \\
\hline $13^{h} 02^{m} 8.40^{s},-31 \operatorname{deg} 56^{\prime} 07.8^{\prime \prime}$ & Aug 17 at $19: 52: 58(0.30)$ & 62 \\
\hline $13^{h} 34^{m} 45.84^{s},-33 \operatorname{deg} 30^{\prime} 03.2^{\prime \prime}$ & Aug 17 at 19:54:32 (0.30) & 57 \\
\hline $13^{h} 36^{m} 15.84^{s},-33 \operatorname{deg} 30^{\prime} 03.2^{\prime \prime}$ & Aug 17 at $19: 56: 11(0.30)$ & 57 \\
\hline $13^{h} 33^{m} 25.92^{s},-32 \operatorname{deg} 52^{\prime} 29.3^{\prime \prime}$ & Aug 17 at $19: 57: 36(0.30)$ & 351 \\
\hline $14^{h} 09^{m} 19.92^{s},-53 \operatorname{deg} 13^{\prime} 32.9^{\prime \prime}$ & Aug 17 at $20: 23: 42(0.32)$ & 17 \\
\hline $14^{h} 21^{m} 11.52^{s},-58 \operatorname{deg} 14^{\prime} 07.1^{\prime \prime}$ & Aug 17 at $20: 25: 41(0.32)$ & 117 \\
\hline $12^{h} 42^{m} 20.16^{s},-12 \operatorname{deg} 50^{\prime} 12.5^{\prime \prime}$ & Aug 17 at $21: 16: 58(0.36)$ & 102 \\
\hline $12^{h} 40^{m} 45.36^{s},-12 \operatorname{deg} 31^{\prime} 25.3^{\prime \prime}$ & Aug 17 at $21: 19: 06(0.36)$ & 117 \\
\hline $12^{h} 41^{m} 3.12^{s},-12 \operatorname{deg} 50^{\prime} 12.5^{\prime \prime}$ & Aug 17 at $21: 21: 37(0.36)$ & 115 \\
\hline
\end{tabular}

${ }^{a}$ Observations were 2017, times in parentheses are days since the GW trigger. 


\begin{tabular}{|c|c|c|}
\hline $\begin{array}{l}\text { Pointing direction } \\
\text { (J2000) }\end{array}$ & $\begin{array}{l}\text { Start time }^{a} \\
\text { (UTC) }\end{array}$ & $\begin{array}{l}\text { Exposure } \\
\text { (s) }\end{array}$ \\
\hline $12^{h} 41^{m} 22.08^{s},-13 \operatorname{deg} 08^{\prime} 59.6^{\prime \prime}$ & Aug 17 at $21: 24: 03(0.36)$ & 115 \\
\hline $12^{h} 42^{m} 39.36^{s},-13 \operatorname{deg} 08^{\prime} 59.6^{\prime \prime}$ & Aug 17 at $21: 26: 28(0.36)$ & 112 \\
\hline $12^{h} 43^{m} 56.40^{s},-13 \operatorname{deg} 08^{\prime} 59.6^{\prime \prime}$ & Aug 17 at $21: 28: 53(0.37)$ & 117 \\
\hline $12^{h} 45^{m} 13.68^{s},-13 \operatorname{deg} 08^{\prime} 59.6^{\prime \prime}$ & Aug 17 at $21: 31: 18(0.37)$ & 120 \\
\hline $12^{h} 45^{m} 34.32^{s},-13 \operatorname{deg} 27^{\prime} 46.8^{\prime \prime}$ & Aug 17 at $21: 33: 43(0.37)$ & 117 \\
\hline $12^{h} 46^{m} 51.60^{s},-13 \operatorname{deg} 27^{\prime} 46.8^{\prime \prime}$ & Aug 17 at $21: 36: 08(0.37)$ & 120 \\
\hline $12^{h} 47^{m} 48.00^{s},-13 \operatorname{deg} 08^{\prime} 59.6^{\prime \prime}$ & Aug 17 at $21: 38: 33(0.37)$ & 122 \\
\hline $12^{h} 37^{m} 27.60^{s},-11 \operatorname{deg} 35^{\prime} 03.8^{\prime \prime}$ & Aug 17 at $22: 51: 56(0.42)$ & 105 \\
\hline $12^{h} 38^{m} 44.40^{s},-11 \operatorname{deg} 35^{\prime} 03.8^{\prime \prime}$ & Aug 17 at $22: 54: 07(0.43)$ & 110 \\
\hline $12^{h} 40^{m} 0.96^{s},-11 \operatorname{deg} 35^{\prime} 03.8^{\prime \prime}$ & Aug 17 at $22: 56: 32(0.43)$ & 117 \\
\hline $12^{h} 41^{m} 17.76^{s},-11 \operatorname{deg} 35^{\prime} 03.8^{\prime \prime}$ & Aug 17 at $22: 58: 56(0.43)$ & 120 \\
\hline $12^{h} 41^{m} 31.20^{s},-11 \operatorname{deg} 53^{\prime} 51.0^{\prime \prime}$ & Aug 17 at $23: 01: 22(0.43)$ & 120 \\
\hline $12^{h} 40^{m} 14.40^{s},-11 \operatorname{deg} 53^{\prime} 51.0^{\prime \prime}$ & Aug 17 at 23:03:47 (0.43) & 112 \\
\hline $12^{h} 38^{m} 57.60^{s},-11 \operatorname{deg} 53^{\prime} 51.0^{\prime \prime}$ & Aug 17 at $23: 06: 11(0.43)$ & 117 \\
\hline $12^{h} 40^{m} 29.28^{s},-12 \operatorname{deg} 12^{\prime} 38.2^{\prime \prime}$ & Aug 17 at $23: 08: 37(0.44)$ & 122 \\
\hline $12^{h} 41^{m} 46.08^{s},-12 \operatorname{deg} 12^{\prime} 38.2^{\prime \prime}$ & Aug 17 at $23: 11: 07(0.44)$ & 115 \\
\hline $12^{h} 43^{m} 19.44^{s},-12 \operatorname{deg} 31^{\prime} 25.3^{\prime \prime}$ & Aug 17 at $23: 13: 33(0.44)$ & 122 \\
\hline $12^{h} 44^{m} 54.24^{s},-12 \operatorname{deg} 50^{\prime} 12.5^{\prime \prime}$ & Aug 17 at $23: 16: 03(0.44)$ & 117 \\
\hline $12^{h} 43^{m} 37.20^{s},-12 \operatorname{deg} 50^{\prime} 12.5^{\prime \prime}$ & Aug 17 at $23: 18: 33(0.44)$ & 120 \\
\hline $12^{h} 39^{m} 48.96^{s},-11 \operatorname{deg} 16^{\prime} 16.7^{\prime \prime}$ & Aug 18 at $00: 29: 03(0.49)$ & 30 \\
\hline $12^{h} 38^{m} 32.40^{s},-11 \operatorname{deg} 16^{\prime} 16.7^{\prime \prime}$ & Aug 18 at $00: 30: 07(0.49)$ & 120 \\
\hline $12^{h} 39^{m} 38.40^{s},-10 \operatorname{deg} 57^{\prime} 29.9^{\prime \prime}$ & Aug 18 at $00: 32: 32(0.49)$ & 117 \\
\hline $12^{h} 40^{m} 54.96^{s},-10 \operatorname{deg} 57^{\prime} 29.9^{\prime \prime}$ & Aug 18 at $00: 35: 02(0.50)$ & 117 \\
\hline $12^{h} 42^{m} 22.32^{s},-11 \operatorname{deg} 16^{\prime} 16.7^{\prime \prime}$ & Aug 18 at $00: 37: 27(0.50)$ & 122 \\
\hline $12^{h} 42^{m} 34.32^{s},-11 \operatorname{deg} 35^{\prime} 03.8^{\prime \prime}$ & Aug 18 at $00: 39: 58(0.50)$ & 117 \\
\hline $12^{h} 42^{m} 48.00^{s},-11 \operatorname{deg} 53^{\prime} 51.0^{\prime \prime}$ & Aug 18 at $00: 42: 23(0.50)$ & 110 \\
\hline $12^{h} 43^{m} 3.12^{s},-12 \operatorname{deg} 12^{\prime} 38.2^{\prime \prime}$ & Aug 18 at $00: 44: 48(0.50)$ & 120 \\
\hline $12^{h} 44^{m} 36.48^{s},-12 \operatorname{deg} 31^{\prime} 25.3^{\prime \prime}$ & Aug 18 at $00: 47: 13(0.50)$ & 125 \\
\hline $12^{h} 45^{m} 36.72^{s},-12 \operatorname{deg} 12^{\prime} 38.2^{\prime \prime}$ & Aug 18 at $00: 49: 44(0.51)$ & 122 \\
\hline $12^{h} 46^{m} 38.40^{s},-11 \operatorname{deg} 53^{\prime} 51.0^{\prime \prime}$ & Aug 18 at $00: 52: 14(0.51)$ & 125 \\
\hline $12^{h} 46^{m} 24.48^{s},-11 \operatorname{deg} 35^{\prime} 03.8^{\prime \prime}$ & Aug 18 at $00: 54: 45(0.51)$ & 112 \\
\hline $12^{h} 47^{m} 41.28^{s},-11 \operatorname{deg} 35^{\prime} 03.8^{\prime \prime}$ & Aug 18 at $00: 57: 10(0.51)$ & 120 \\
\hline $12^{h} 44^{m} 19.92^{s},-10 \operatorname{deg} 01^{\prime} 08.4^{\prime \prime}$ & Aug 18 at $02: 04: 38(0.56)$ & 57 \\
\hline $12^{h} 45^{m} 42.96^{s},-10 \operatorname{deg} 19^{\prime} 55.6^{\prime \prime}$ & Aug 18 at $02: 06: 07(0.56)$ & 117 \\
\hline $12^{h} 45^{m} 36.24^{s},-10 \operatorname{deg} 01^{\prime} 08.4^{\prime \prime}$ & Aug 18 at 02:08:38 (0.56) & 110 \\
\hline $12^{h} 46^{m} 52.56^{s},-10 \operatorname{deg} 01^{\prime} 08.4^{\prime \prime}$ & Aug 18 at $02: 11: 03(0.56)$ & 115 \\
\hline
\end{tabular}

${ }^{a}$ Observations were 2017, times in parentheses are days since the GW trigger. 


\begin{tabular}{|c|c|c|}
\hline $\begin{array}{l}\text { Pointing direction } \\
\text { (J2000) }\end{array}$ & $\begin{array}{l}\text { Start time }^{a} \\
\text { (UTC) }\end{array}$ & $\begin{array}{l}\text { Exposure } \\
\text { (s) }\end{array}$ \\
\hline $12^{h} 46^{m} 59.52^{s},-10 \operatorname{deg} 19^{\prime} 55.6^{\prime \prime}$ & Aug 18 at $02: 13: 28(0.56)$ & 117 \\
\hline $12^{h} 45^{m} 51.36^{s},-10 \operatorname{deg} 38^{\prime} 42.7^{\prime \prime}$ & Aug 18 at $02: 15: 53(0.57)$ & 117 \\
\hline $12^{h} 46^{m} 0.96^{s},-10 \operatorname{deg} 57^{\prime} 29.9^{\prime \prime}$ & Aug 18 at $02: 18: 23(0.57)$ & 110 \\
\hline $12^{h} 44^{m} 55.44^{s},-11 \operatorname{deg} 16^{\prime} 16.7^{\prime \prime}$ & Aug 18 at $02: 20: 48(0.57)$ & 117 \\
\hline $12^{h} 43^{m} 51.12^{s},-11 \operatorname{deg} 35^{\prime} 03.8^{\prime \prime}$ & Aug 18 at $02: 23: 19(0.57)$ & 120 \\
\hline $12^{h} 45^{m} 21.60^{s},-11 \operatorname{deg} 53^{\prime} 51.0^{\prime \prime}$ & Aug 18 at $02: 25: 49(0.57)$ & 122 \\
\hline $12^{h} 46^{m} 53.76^{s},-12 \operatorname{deg} 12^{\prime} 38.2^{\prime \prime}$ & Aug 18 at $02: 28: 20(0.57)$ & 122 \\
\hline $12^{h} 47^{m} 55.20^{s},-11 \operatorname{deg} 53^{\prime} 51.0^{\prime \prime}$ & Aug 18 at $02: 30: 49(0.58)$ & 125 \\
\hline $12^{h} 49^{m} 12.00^{s},-11 \operatorname{deg} 53^{\prime} 51.0^{\prime \prime}$ & Aug 18 at $02: 33: 21(0.58)$ & 117 \\
\hline $12^{h} 50^{m} 44.40^{s},-12 \operatorname{deg} 12^{\prime} 38.2^{\prime \prime}$ & Aug 18 at $02: 35: 45(0.58)$ & 122 \\
\hline $13^{h} 07^{m} 0.96^{s},-23 \operatorname{deg} 47^{\prime} 42.4^{\prime \prime}$ & Aug 18 at $02: 38: 17(0.58)$ & 112 \\
\hline $12^{h} 44^{m} 4.80^{s},-11 \operatorname{deg} 53^{\prime} 51.0^{\prime \prime}$ & Aug 18 at 04:11:06 (0.65) & 72 \\
\hline $13^{h} 08^{m} 23.04^{s},-23 \operatorname{deg} 47^{\prime} 42.4^{\prime \prime}$ & Aug 18 at 04:13:17 (0.65) & 115 \\
\hline $13^{h} 20^{m} 55.68^{s},-27 \operatorname{deg} 33^{\prime} 07.9^{\prime \prime}$ & Aug 18 at 04:16:04 (0.65) & 117 \\
\hline $13^{h} 58^{m} 42.96^{s},-48 \operatorname{deg} 31^{\prime} 45.8^{\prime \prime}$ & Aug 18 at $04: 18: 39(0.65)$ & 117 \\
\hline $12^{h} 37^{m} 40.80^{s},-11 \operatorname{deg} 53^{\prime} 51.0^{\prime \prime}$ & Aug 18 at $05: 15: 41(0.69)$ & 65 \\
\hline $12^{h} 37^{m} 55.44^{s},-12 \operatorname{deg} 12^{\prime} 38.2^{\prime \prime}$ & Aug 18 at $05: 17: 13(0.69)$ & 112 \\
\hline $12^{h} 39^{m} 28.56^{s},-12 \operatorname{deg} 31^{\prime} 25.3^{\prime \prime}$ & Aug 18 at $05: 19: 38(0.69)$ & 120 \\
\hline $12^{h} 39^{m} 46.08^{s},-12 \operatorname{deg} 50^{\prime} 12.5^{\prime \prime}$ & Aug 18 at $05: 22: 08(0.70)$ & 112 \\
\hline $12^{h} 40^{m} 5.04^{s},-13 \operatorname{deg} 08^{\prime} 59.6^{\prime \prime}$ & Aug 18 at $05: 24: 32(0.70)$ & 117 \\
\hline $12^{h} 40^{m} 25.20^{s},-13 \operatorname{deg} 27^{\prime} 46.8^{\prime \prime}$ & Aug 18 at $05: 26: 57(0.70)$ & 117 \\
\hline $12^{h} 41^{m} 42.48^{s},-13 \operatorname{deg} 27^{\prime} 46.8^{\prime \prime}$ & Aug 18 at $05: 29: 23(0.70)$ & 112 \\
\hline $12^{h} 42^{m} 59.76^{s},-13 \operatorname{deg} 27^{\prime} 46.8^{\prime \prime}$ & Aug 18 at $05: 31: 48(0.70)$ & 112 \\
\hline $12^{h} 44^{m} 17.04^{s},-13 \operatorname{deg} 27^{\prime} 46.8^{\prime \prime}$ & Aug 18 at $05: 34: 13(0.70)$ & 120 \\
\hline $12^{h} 44^{m} 39.12^{s},-13 \operatorname{deg} 46^{\prime} 34.0^{\prime \prime}$ & Aug 18 at $05: 36: 38(0.71)$ & 110 \\
\hline $12^{h} 45^{m} 56.64^{s},-13 \operatorname{deg} 46^{\prime} 34.0^{\prime \prime}$ & Aug 18 at $05: 39: 03(0.71)$ & 117 \\
\hline $12^{h} 47^{m} 13.92^{s},-13 \operatorname{deg} 46^{\prime} 34.0^{\prime \prime}$ & Aug 18 at $05: 41: 28(0.71)$ & 112 \\
\hline $12^{h} 47^{m} 37.68^{s},-14 \operatorname{deg} 05^{\prime} 21.1^{\prime \prime}$ & Aug 18 at $05: 43: 53(0.71)$ & 115 \\
\hline $12^{h} 48^{m} 2.88^{s},-14 \operatorname{deg} 24^{\prime} 08.3^{\prime \prime}$ & Aug 18 at $05: 46: 18(0.71)$ & 120 \\
\hline $12^{h} 53^{m} 35.28^{s},-16 \operatorname{deg} 54^{\prime} 25.2^{\prime \prime}$ & Aug 18 at $05: 48: 43(0.71)$ & 117 \\
\hline $13^{h} 24^{m} 53.28^{s},-30 \operatorname{deg} 22^{\prime} 12.0^{\prime \prime}$ & Aug 18 at 05:51:17 (0.72) & 117 \\
\hline $14^{h} 10^{m} 39.60^{s},-52 \operatorname{deg} 17^{\prime} 11.4^{\prime \prime}$ & Aug 18 at $05: 54: 07(0.72)$ & 117 \\
\hline $14^{h} 09^{m} 47.28^{s},-51 \operatorname{deg} 58^{\prime} 24.2^{\prime \prime}$ & Aug 18 at 05:57:07 (0.72) & 112 \\
\hline $12^{h} 40^{m} 17.28^{s},-14 \operatorname{deg} 24^{\prime} 08.3^{\prime \prime}$ & Aug 18 at $06: 51: 12(0.76)$ & 85 \\
\hline $12^{h} 40^{m} 43.20^{s},-14 \operatorname{deg} 42^{\prime} 55.4^{\prime \prime}$ & Aug 18 at 06:53:07 (0.76) & 117 \\
\hline $12^{h} 42^{m} 0.96^{s},-14 \operatorname{deg} 42^{\prime} 55.4^{\prime \prime}$ & Aug 18 at $06: 55: 31(0.76)$ & 117 \\
\hline
\end{tabular}

${ }^{a}$ Observations were 2017, times in parentheses are days since the GW trigger. 


\begin{tabular}{|c|c|c|}
\hline $\begin{array}{l}\text { Pointing direction } \\
\text { (J2000) }\end{array}$ & $\begin{array}{l}\text { Start time }^{a} \\
\text { (UTC) }\end{array}$ & $\begin{array}{l}\text { Exposure } \\
\text { (s) }\end{array}$ \\
\hline $12^{h} 42^{m} 52.56^{s},-14 \operatorname{deg} 24^{\prime} 08.3^{\prime \prime}$ & Aug 18 at $06: 57: 57(0.76)$ & 125 \\
\hline $12^{h} 43^{m} 45.12^{s},-14 \operatorname{deg} 05^{\prime} 21.1^{\prime \prime}$ & Aug 18 at $07: 00: 27(0.76)$ & 125 \\
\hline $12^{h} 45^{m} 2.64^{s},-14 \operatorname{deg} 05^{\prime} 21.1^{\prime \prime}$ & Aug 18 at 07:02:58 (0.77) & 120 \\
\hline $12^{h} 45^{m} 27.60^{s},-14 \operatorname{deg} 24^{\prime} 08.3^{\prime \prime}$ & Aug 18 at $07: 05: 23(0.77)$ & 117 \\
\hline $12^{h} 45^{m} 54.00^{s},-14 \operatorname{deg} 42^{\prime} 55.4^{\prime \prime}$ & Aug 18 at $07: 07: 48(0.77)$ & 112 \\
\hline $12^{h} 46^{m} 45.12^{s},-14 \operatorname{deg} 24^{\prime} 08.3^{\prime \prime}$ & Aug 18 at $07: 10: 13(0.77)$ & 120 \\
\hline $12^{h} 47^{m} 11.76^{s},-14 \operatorname{deg} 42^{\prime} 55.4^{\prime \prime}$ & Aug 18 at $07: 12: 43(0.77)$ & 112 \\
\hline $12^{h} 48^{m} 29.28^{s},-14 \operatorname{deg} 42^{\prime} 55.4^{\prime \prime}$ & Aug 18 at $07: 15: 08(0.77)$ & 115 \\
\hline $12^{h} 49^{m} 20.40^{s},-14 \operatorname{deg} 24^{\prime} 08.3^{\prime \prime}$ & Aug 18 at $07: 17: 33(0.78)$ & 127 \\
\hline $12^{h} 50^{m} 37.92^{s},-14 \operatorname{deg} 24^{\prime} 08.3^{\prime \prime}$ & Aug 18 at $07: 20: 04(0.78)$ & 117 \\
\hline $12^{h} 49^{m} 47.04^{s},-14 \operatorname{deg} 42^{\prime} 55.4^{\prime \prime}$ & Aug 18 at $07: 22: 29(0.78)$ & 122 \\
\hline $12^{h} 57^{m} 38.40^{s},-19 \operatorname{deg} 43^{\prime} 29.6^{\prime \prime}$ & Aug 18 at 07:24:59 (0.78) & 117 \\
\hline $13^{h} 26^{m} 25.68^{s},-33 \operatorname{deg} 48^{\prime} 50.4^{\prime \prime}$ & Aug 18 at $07: 27: 36(0.78)$ & 117 \\
\hline $13^{h} 56^{m} 52.32^{s},-44 \operatorname{deg} 08^{\prime} 46.0^{\prime \prime}$ & Aug 18 at $07: 30: 26(0.78)$ & 117 \\
\hline $14^{h} 15^{m} 41.76^{s},-52 \operatorname{deg} 35^{\prime} 58.6^{\prime \prime}$ & Aug 18 at $07: 33: 12(0.79)$ & 180 \\
\hline $12^{h} 38^{m} 21.84^{s},-10 \operatorname{deg} 57^{\prime} 29.9^{\prime \prime}$ & Aug 18 at $08: 25: 49(0.82)$ & 115 \\
\hline $12^{h} 39^{m} 29.04^{s},-10 \operatorname{deg} 38^{\prime} 42.7^{\prime \prime}$ & Aug 18 at $08: 28: 12(0.82)$ & 127 \\
\hline $12^{h} 40^{m} 45.60^{s},-10 \operatorname{deg} 38^{\prime} 42.7^{\prime \prime}$ & Aug 18 at $08: 30: 43(0.83)$ & 120 \\
\hline $12^{h} 39^{m} 21.12^{s},-10 \operatorname{deg} 19^{\prime} 55.6^{\prime \prime}$ & Aug 18 at 08:33:08 (0.83) & 122 \\
\hline $12^{h} 38^{m} 12.72^{s},-10 \operatorname{deg} 38^{\prime} 42.7^{\prime \prime}$ & Aug 18 at $08: 35: 39(0.83)$ & 115 \\
\hline $12^{h} 38^{m} 4.80^{s},-10 \operatorname{deg} 19^{\prime} 55.6^{\prime \prime}$ & Aug 18 at $08: 38: 09(0.83)$ & 115 \\
\hline $12^{h} 39^{m} 14.64^{s},-10 \operatorname{deg} 01^{\prime} 08.4^{\prime \prime}$ & Aug 18 at $08: 40: 34(0.83)$ & 125 \\
\hline $12^{h} 37^{m} 58.32^{s},-10 \operatorname{deg} 01^{\prime} 08.4^{\prime \prime}$ & Aug 18 at 08:43:05 (0.83) & 117 \\
\hline $12^{h} 37^{m} 53.28^{s},-09 \operatorname{deg} 42^{\prime} 21.2^{\prime \prime}$ & Aug 18 at $08: 45: 30(0.84)$ & 117 \\
\hline $12^{h} 36^{m} 42.00^{s},-10 \operatorname{deg} 01^{\prime} 08.4^{\prime \prime}$ & Aug 18 at $08: 47: 55(0.84)$ & 117 \\
\hline $12^{h} 36^{m} 48.48^{s},-10 \operatorname{deg} 19^{\prime} 55.6^{\prime \prime}$ & Aug 18 at $08: 50: 24(0.84)$ & 117 \\
\hline $12^{h} 36^{m} 56.16^{s},-10 \operatorname{deg} 38^{\prime} 42.7^{\prime \prime}$ & Aug 18 at $08: 52: 50(0.84)$ & 112 \\
\hline $12^{h} 37^{m} 5.28^{s},-10 \operatorname{deg} 57^{\prime} 29.9^{\prime \prime}$ & Aug 18 at $08: 55: 15(0.84)$ & 117 \\
\hline $12^{h} 35^{m} 39.60^{s},-10 \operatorname{deg} 38^{\prime} 42.7^{\prime \prime}$ & Aug 18 at $08: 57: 39(0.84)$ & 120 \\
\hline $13^{h} 06^{m} 52.08^{s},-24 \operatorname{deg} 06^{\prime} 29.5^{\prime \prime}$ & Aug 18 at 09:00:11 (0.85) & 115 \\
\hline $13^{h} 20^{m} 55.92^{s},-29 \operatorname{deg} 25^{\prime} 50.5^{\prime \prime}$ & Aug 18 at 09:03:01 (0.85) & 117 \\
\hline $13^{h} 56^{m} 48.72^{s},-43 \operatorname{deg} 49^{\prime} 58.8^{\prime \prime}$ & Aug 18 at $09: 05: 39(0.85)$ & 117 \\
\hline $14^{h} 08^{m} 13.44^{s},-53 \operatorname{deg} 32^{\prime} 20.0^{\prime \prime}$ & Aug 18 at 09:08:30 (0.85) & 160 \\
\hline $12^{h} 45^{m} 30.72^{s},-09 \operatorname{deg} 42^{\prime} 21.2^{\prime \prime}$ & Aug 18 at 10:01:47 (0.89) & 105 \\
\hline $12^{h} 46^{m} 42.72^{s},-09 \operatorname{deg} 23^{\prime} 34.0^{\prime \prime}$ & Aug 18 at 10:04:07 (0.89) & 125 \\
\hline $12^{h} 46^{m} 47.04^{s},-09 \operatorname{deg} 42^{\prime} 21.2^{\prime \prime}$ & Aug 18 at 10:06:38 (0.89) & 115 \\
\hline
\end{tabular}

${ }^{a}$ Observations were 2017, times in parentheses are days since the GW trigger. 


\begin{tabular}{|c|c|c|}
\hline $\begin{array}{l}\text { Pointing direction } \\
\text { (J2000) }\end{array}$ & $\begin{array}{l}\text { Start time }^{a} \\
\text { (UTC) }\end{array}$ & $\begin{array}{l}\text { Exposure } \\
\text { (s) }\end{array}$ \\
\hline $12^{h} 48^{m} 8.88^{s},-10 \operatorname{deg} 01^{\prime} 08.4^{\prime \prime}$ & Aug 18 at 10:09:03 (0.89) & 125 \\
\hline $12^{h} 48^{m} 15.84^{s},-10 \operatorname{deg} 19^{\prime} 55.6^{\prime \prime}$ & Aug 18 at $10: 11: 33(0.90)$ & 120 \\
\hline $12^{h} 49^{m} 40.80^{s},-10 \operatorname{deg} 38^{\prime} 42.7^{\prime \prime}$ & Aug 18 at $10: 13: 57(0.90)$ & 120 \\
\hline $12^{h} 49^{m} 50.64^{s},-10 \operatorname{deg} 57^{\prime} 29.9^{\prime \prime}$ & Aug 18 at $10: 16: 29(0.90)$ & 115 \\
\hline $12^{h} 51^{m} 7.20^{s},-10 \operatorname{deg} 57^{\prime} 29.9^{\prime \prime}$ & Aug 18 at $10: 18: 54(0.90)$ & 112 \\
\hline $12^{h} 50^{m} 1.92^{s},-11 \operatorname{deg} 16^{\prime} 16.7^{\prime \prime}$ & Aug 18 at 10:21:19(0.90) & 115 \\
\hline $12^{h} 48^{m} 57.84^{s},-11 \operatorname{deg} 35^{\prime} 03.8^{\prime \prime}$ & Aug 18 at $10: 23: 49(0.90)$ & 115 \\
\hline $12^{h} 50^{m} 28.80^{s},-11 \operatorname{deg} 53^{\prime} 51.0^{\prime \prime}$ & Aug 18 at $10: 26: 20(0.91)$ & 117 \\
\hline $12^{h} 49^{m} 27.36^{s},-12 \operatorname{deg} 12^{\prime} 38.2^{\prime \prime}$ & Aug 18 at $10: 28: 50(0.91)$ & 122 \\
\hline $12^{h} 51^{m} 1.20^{s},-12 \operatorname{deg} 31^{\prime} 25.3^{\prime \prime}$ & Aug 18 at $10: 31: 21(0.91)$ & 122 \\
\hline $12^{h} 50^{m} 2.64^{s},-12 \operatorname{deg} 50^{\prime} 12.5^{\prime \prime}$ & Aug 18 at $10: 33: 52(0.91)$ & 122 \\
\hline $13^{h} 08^{m} 14.40^{s},-24 \operatorname{deg} 06^{\prime} 29.5^{\prime \prime}$ & Aug 18 at $10: 36: 22(0.91)$ & 115 \\
\hline $13^{h} 32^{m} 35.28^{s},-33 \operatorname{deg} 11^{\prime} 16.4^{\prime \prime}$ & Aug 18 at 10:39:08 (0.92) & 115 \\
\hline $13^{h} 55^{m} 12.48^{s},-44 \operatorname{deg} 27^{\prime} 33.1^{\prime \prime}$ & Aug 18 at $10: 41: 51(0.92)$ & 117 \\
\hline $14^{h} 07^{m} 14.40^{s},-53 \operatorname{deg} 13^{\prime} 32.9^{\prime \prime}$ & Aug 18 at $10: 44: 37(0.92)$ & 155 \\
\hline $12^{h} 45^{m} 53.28^{s},-12 \operatorname{deg} 31^{\prime} 25.3^{\prime \prime}$ & Aug 18 at $11: 38: 58(0.96)$ & 47 \\
\hline $12^{h} 46^{m} 11.28^{s},-12 \operatorname{deg} 50^{\prime} 12.5^{\prime \prime}$ & Aug 18 at $11: 40: 13(0.96)$ & 117 \\
\hline $12^{h} 46^{m} 30.72^{s},-13 \operatorname{deg} 08^{\prime} 59.6^{\prime \prime}$ & Aug 18 at $11: 42: 38(0.96)$ & 115 \\
\hline $12^{h} 47^{m} 28.32^{s},-12 \operatorname{deg} 50^{\prime} 12.5^{\prime \prime}$ & Aug 18 at $11: 45: 03(0.96)$ & 125 \\
\hline $12^{h} 48^{m} 45.60^{s},-12 \operatorname{deg} 50^{\prime} 12.5^{\prime \prime}$ & Aug 18 at $11: 47: 33(0.96)$ & 115 \\
\hline $12^{h} 49^{m} 5.04^{s},-13 \operatorname{deg} 08^{\prime} 59.6^{\prime \prime}$ & Aug 18 at $11: 49: 58(0.96)$ & 117 \\
\hline $12^{h} 49^{m} 26.16^{s},-13 \operatorname{deg} 27^{\prime} 46.8^{\prime \prime}$ & Aug 18 at $11: 52: 22(0.97)$ & 117 \\
\hline $12^{h} 50^{m} 43.44^{s},-13 \operatorname{deg} 27^{\prime} 46.8^{\prime \prime}$ & Aug 18 at $11: 54: 48(0.97)$ & 112 \\
\hline $12^{h} 50^{m} 22.32^{s},-13 \operatorname{deg} 08^{\prime} 59.6^{\prime \prime}$ & Aug 18 at $11: 57: 13(0.97)$ & 120 \\
\hline $12^{h} 51^{m} 39.36^{s},-13 \operatorname{deg} 08^{\prime} 59.6^{\prime \prime}$ & Aug 18 at $11: 59: 38(0.97)$ & 112 \\
\hline $12^{h} 52^{m} 0.72^{s},-13 \operatorname{deg} 27^{\prime} 46.8^{\prime \prime}$ & Aug 18 at $12: 02: 02(0.97)$ & 112 \\
\hline $12^{h} 53^{m} 18.00^{s},-13 \operatorname{deg} 27^{\prime} 46.8^{\prime \prime}$ & Aug 18 at $12: 04: 28(0.97)$ & 110 \\
\hline $12^{h} 52^{m} 56.64^{s},-13 \operatorname{deg} 08^{\prime} 59.6^{\prime \prime}$ & Aug 18 at $12: 06: 53(0.98)$ & 110 \\
\hline $12^{h} 53^{m} 53.76^{s},-12 \operatorname{deg} 50^{\prime} 12.5^{\prime \prime}$ & Aug 18 at $12: 09: 18(0.98)$ & 122 \\
\hline $13^{h} 09^{m} 54.72^{s},-23 \operatorname{deg} 28^{\prime} 55.2^{\prime \prime}$ & Aug 18 at $12: 11: 49(0.98)$ & 115 \\
\hline $13^{h} 25^{m} 56.88^{s},-30 \operatorname{deg} 03^{\prime} 24.8^{\prime \prime}$ & Aug 18 at $12: 14: 33(0.98)$ & 117 \\
\hline $14^{h} 09^{m} 22.56^{s},-51 \operatorname{deg} 02^{\prime} 03.1^{\prime \prime}$ & Aug 18 at $12: 17: 13(0.98)$ & 117 \\
\hline $14^{h} 10^{m} 9.36^{s},-51 \operatorname{deg} 20^{\prime} 49.9^{\prime \prime}$ & Aug 18 at $12: 20: 11(0.99)$ & 120 \\
\hline $12^{h} 34^{m} 4.80^{s},-12 \operatorname{deg} 12^{\prime} 38.2^{\prime \prime}$ & Aug 18 at 13:13:59 (1.0) & 37 \\
\hline $12^{h} 52^{m} 34.08^{s},-15 \operatorname{deg} 39^{\prime} 16.6^{\prime \prime}$ & Aug 18 at 13:15:07 (1.0) & 117 \\
\hline $12^{h} 52^{m} 2.88^{s},-15 \operatorname{deg} 20^{\prime} 29.8^{\prime \prime}$ & Aug 18 at 13:17:44 (1.0) & 120 \\
\hline
\end{tabular}

${ }^{a}$ Observations were 2017, times in parentheses are days since the GW trigger. 


\begin{tabular}{|c|c|c|}
\hline $\begin{array}{l}\text { Pointing direction } \\
\text { (J2000) }\end{array}$ & $\begin{array}{l}\text { Start time }^{a} \\
\text { (UTC) }\end{array}$ & $\begin{array}{c}\text { Exposure } \\
\text { (s) }\end{array}$ \\
\hline $12^{h} 53^{m} 20.64^{s},-15 \operatorname{deg} 20^{\prime} 29.8^{\prime \prime}$ & Aug 18 at $13: 20: 09(1.0)$ & 120 \\
\hline $12^{h} 53^{m} 52.08^{s},-15 \operatorname{deg} 39^{\prime} 16.6^{\prime \prime}$ & Aug 18 at $13: 22: 33(1.0)$ & 115 \\
\hline $12^{h} 54^{m} 24.96^{s},-15 \operatorname{deg} 58^{\prime} 03.7^{\prime \prime}$ & Aug 18 at $13: 24: 59(1.0)$ & 117 \\
\hline $12^{h} 54^{m} 59.28^{s},-16 \operatorname{deg} 16^{\prime} 50.9^{\prime \prime}$ & Aug 18 at 13:27:23 (1.0) & 112 \\
\hline $13^{h} 38^{m} 11.04^{s},-36 \operatorname{deg} 00^{\prime} 20.5^{\prime \prime}$ & Aug 18 at 13:52:35 (1.0) & 70 \\
\hline $14^{h} 00^{m} 9.12^{s},-48 \operatorname{deg} 12^{\prime} 58.7^{\prime \prime}$ & Aug 18 at $13: 54: 40(1.1)$ & 117 \\
\hline $14^{h} 18^{m} 48.72^{s},-58 \operatorname{deg} 14^{\prime} 07.1^{\prime \prime}$ & Aug 18 at $13: 57: 26(1.1)$ & 117 \\
\hline $12^{h} 48^{m} 34.08^{s},-10 \operatorname{deg} 57^{\prime} 29.9^{\prime \prime}$ & Aug 18 at 14:59:06 (1.1) & 55 \\
\hline $12^{h} 47^{m} 17.52^{s},-10 \operatorname{deg} 57^{\prime} 29.9^{\prime \prime}$ & Aug 18 at 15:00:53 (1.1) & 117 \\
\hline $12^{h} 47^{m} 7.92^{s},-10 \operatorname{deg} 38^{\prime} 42.7^{\prime \prime}$ & Aug 18 at 15:03:18 (1.1) & 117 \\
\hline $12^{h} 56^{m} 51.12^{s},-17 \operatorname{deg} 13^{\prime} 12.4^{\prime \prime}$ & Aug 18 at $15: 05: 43(1.1)$ & 117 \\
\hline $12^{h} 57^{m} 31.68^{s},-17 \operatorname{deg} 31^{\prime} 59.5^{\prime \prime}$ & Aug 18 at 15:08:22 (1.1) & 120 \\
\hline $12^{h} 58^{m} 50.40^{s},-17 \operatorname{deg} 31^{\prime} 59.5^{\prime \prime}$ & Aug 18 at $15: 10: 47(1.1)$ & 117 \\
\hline $12^{h} 58^{m} 9.84^{s},-17 \operatorname{deg} 13^{\prime} 12.4^{\prime \prime}$ & Aug 18 at $15: 13: 12(1.1)$ & 117 \\
\hline $12^{h} 58^{m} 49.44^{s},-16 \operatorname{deg} 54^{\prime} 25.2^{\prime \prime}$ & Aug 18 at $15: 15: 37$ (1.1) & 120 \\
\hline $12^{h} 58^{m} 11.76^{s},-16 \operatorname{deg} 35^{\prime} 38.0^{\prime \prime}$ & Aug 18 at $15: 18: 02(1.1)$ & 112 \\
\hline $12^{h} 57^{m} 30.96^{s},-16 \operatorname{deg} 54^{\prime} 25.2^{\prime \prime}$ & Aug 18 at 15:20:27 (1.1) & 112 \\
\hline $12^{h} 56^{m} 12.24^{s},-16 \operatorname{deg} 54^{\prime} 25.2^{\prime \prime}$ & Aug 18 at $15: 22: 52(1.1)$ & 115 \\
\hline $13^{h} 25^{m} 35.04^{s},-29 \operatorname{deg} 44^{\prime} 37.7^{\prime \prime}$ & Aug 18 at $15: 25: 17$ (1.1) & 115 \\
\hline $13^{h} 50^{m} 56.40^{s},-40 \operatorname{deg} 42^{\prime} 07.6^{\prime \prime}$ & Aug 18 at $15: 28: 05$ (1.1) & 117 \\
\hline $14^{h} 02^{m} 42.00^{s},-50 \operatorname{deg} 43^{\prime} 16.0^{\prime \prime}$ & Aug 18 at $15: 30: 51$ (1.1) & 117 \\
\hline $12^{h} 43^{m} 21.84^{s},-13 \operatorname{deg} 46^{\prime} 34.0^{\prime \prime}$ & Aug 18 at $16: 20: 57(1.2)$ & 82 \\
\hline $12^{h} 42^{m} 4.32^{s},-13 \operatorname{deg} 46^{\prime} 34.0^{\prime \prime}$ & Aug 18 at $16: 24: 42(1.2)$ & 117 \\
\hline $12^{h} 42^{m} 27.84^{s},-14 \operatorname{deg} 05^{\prime} 21.1^{\prime \prime}$ & Aug 18 at $16: 27: 07$ (1.2) & 112 \\
\hline $12^{h} 41^{m} 10.32^{s},-14 \operatorname{deg} 05^{\prime} 21.1^{\prime \prime}$ & Aug 18 at $16: 29: 32(1.2)$ & 112 \\
\hline $12^{h} 40^{m} 47.04^{s},-13 \operatorname{deg} 46^{\prime} 34.0^{\prime \prime}$ & Aug 18 at $16: 31: 57(1.2)$ & 120 \\
\hline $13^{h} 07^{m} 11.04^{s},-23 \operatorname{deg} 28^{\prime} 55.2^{\prime \prime}$ & Aug 18 at $16: 34: 22(1.2)$ & 115 \\
\hline $13^{h} 08^{m} 32.88^{s},-23 \operatorname{deg} 28^{\prime} 55.2^{\prime \prime}$ & Aug 18 at $16: 37: 07$ (1.2) & 117 \\
\hline $13^{h} 10^{m} 5.76^{s},-23 \operatorname{deg} 10^{\prime} 08.0^{\prime \prime}$ & Aug 18 at $16: 39: 31(1.2)$ & 122 \\
\hline $13^{h} 08^{m} 44.16^{s},-23 \operatorname{deg} 10^{\prime} 08.0^{\prime \prime}$ & Aug 18 at $16: 42: 03(1.2)$ & 120 \\
\hline $13^{h} 07^{m} 35.28^{s},-22 \operatorname{deg} 51^{\prime} 20.9^{\prime \prime}$ & Aug 18 at $16: 44: 28(1.2)$ & 125 \\
\hline $13^{h} 06^{m} 13.68^{s},-22 \operatorname{deg} 51^{\prime} 20.9^{\prime \prime}$ & Aug 18 at $16: 46: 57(1.2)$ & 112 \\
\hline $13^{h} 06^{m} 28.08^{s},-22 \operatorname{deg} 32^{\prime} 33.7^{\prime \prime}$ & Aug 18 at $16: 49: 23(1.2)$ & 120 \\
\hline $13^{h} 05^{m} 6.72^{s},-22 \operatorname{deg} 32^{\prime} 33.7^{\prime \prime}$ & Aug 18 at $16: 51: 47(1.2)$ & 117 \\
\hline $13^{h} 05^{m} 22.56^{s},-22 \operatorname{deg} 13^{\prime} 46.6^{\prime \prime}$ & Aug 18 at $16: 54: 13$ (1.2) & 117 \\
\hline $13^{h} 06^{m} 43.68^{s},-22 \operatorname{deg} 13^{\prime} 46.6^{\prime \prime}$ & Aug 18 at $16: 56: 38$ (1.2) & 110 \\
\hline
\end{tabular}

${ }^{a}$ Observations were 2017, times in parentheses are days since the GW trigger. 


\begin{tabular}{|c|c|c|}
\hline $\begin{array}{l}\text { Pointing direction } \\
\text { (J2000) }\end{array}$ & $\begin{array}{l}\text { Start time }^{a} \\
\text { (UTC) }\end{array}$ & $\begin{array}{c}\text { Exposure } \\
\text { (s) }\end{array}$ \\
\hline $13^{h} 07^{m} 49.44^{s},-22 \operatorname{deg} 32^{\prime} 33.7^{\prime \prime}$ & Aug 18 at 16:59:03 (1.2) & 117 \\
\hline $13^{h} 23^{m} 19.92^{s},-32 \operatorname{deg} 14^{\prime} 55.0^{\prime \prime}$ & Aug 18 at 17:01:34 (1.2) & 117 \\
\hline $13^{h} 56^{m} 49.44^{s},-48 \operatorname{deg} 31^{\prime} 45.8^{\prime \prime}$ & Aug 18 at 17:04:17 (1.2) & 117 \\
\hline $14^{h} 15^{m} 36.96^{s},-53 \operatorname{deg} 51^{\prime} 07.2^{\prime \prime}$ & Aug 18 at 17:07:10 (1.2) & 117 \\
\hline $12^{h} 41^{m} 42.00^{s},-09 \operatorname{deg} 42^{\prime} 21.2^{\prime \prime}$ & Aug 18 at 18:00:01 (1.2) & 100 \\
\hline $13^{h} 11^{m} 7.20^{s},-23 \operatorname{deg} 47^{\prime} 42.4^{\prime \prime}$ & Aug 18 at 18:02:06 (1.2) & 112 \\
\hline $13^{h} 09^{m} 36.72^{s},-24 \operatorname{deg} 06^{\prime} 29.5^{\prime \prime}$ & Aug 18 at 18:04:56 (1.2) & 127 \\
\hline $13^{h} 09^{m} 29.76^{s},-24 \operatorname{deg} 25^{\prime} 16.7^{\prime \prime}$ & Aug 18 at 18:07:28 (1.2) & 117 \\
\hline $13^{h} 10^{m} 59.04^{s},-24 \operatorname{deg} 06^{\prime} 29.5^{\prime \prime}$ & Aug 18 at 18:09:53 (1.2) & 120 \\
\hline $13^{h} 09^{m} 45.12^{s},-23 \operatorname{deg} 47^{\prime} 42.4^{\prime \prime}$ & Aug 18 at $18: 12: 23(1.2)$ & 120 \\
\hline $13^{h} 11^{m} 16.80^{s},-23 \operatorname{deg} 28^{\prime} 55.2^{\prime \prime}$ & Aug 18 at $18: 14: 54(1.2)$ & 120 \\
\hline $13^{h} 11^{m} 27.60^{s},-23 \operatorname{deg} 10^{\prime} 08.0^{\prime \prime}$ & Aug 18 at $18: 17: 25(1.2)$ & 117 \\
\hline $13^{h} 10^{m} 18.24^{s},-22 \operatorname{deg} 51^{\prime} 20.9^{\prime \prime}$ & Aug 18 at $18: 19: 49(1.2)$ & 122 \\
\hline $13^{h} 08^{m} 56.64^{s},-22 \operatorname{deg} 51^{\prime} 20.9^{\prime \prime}$ & Aug 18 at $18: 22: 20(1.2)$ & 120 \\
\hline $13^{h} 07^{m} 22.32^{s},-23 \operatorname{deg} 10^{\prime} 08.0^{\prime \prime}$ & Aug 18 at $18: 24: 45(1.2)$ & 122 \\
\hline $13^{h} 05^{m} 48.96^{s},-23 \operatorname{deg} 28^{\prime} 55.2^{\prime \prime}$ & Aug 18 at $18: 27: 16(1.2)$ & 125 \\
\hline $13^{h} 05^{m} 38.64^{s},-23 \operatorname{deg} 47^{\prime} 42.4^{\prime \prime}$ & Aug 18 at 18:29:47 (1.2) & 117 \\
\hline $13^{h} 05^{m} 29.76^{s},-24 \operatorname{deg} 06^{\prime} 29.5^{\prime \prime}$ & Aug 18 at $18: 32: 12(1.2)$ & 117 \\
\hline $13^{h} 06^{m} 44.88^{s},-24 \operatorname{deg} 25^{\prime} 16.7^{\prime \prime}$ & Aug 18 at $18: 34: 36(1.2)$ & 125 \\
\hline $13^{h} 31^{m} 5.52^{s},-33 \operatorname{deg} 11^{\prime} 16.4^{\prime \prime}$ & Aug 18 at 18:37:07 (1.2) & 117 \\
\hline $13^{h} 43^{m} 39.84^{s},-41 \operatorname{deg} 38^{\prime} 29.0^{\prime \prime}$ & Aug 18 at 18:39:50 (1.2) & 115 \\
\hline $14^{h} 05^{m} 23.28^{s},-52 \operatorname{deg} 35^{\prime} 58.6^{\prime \prime}$ & Aug 18 at $18: 42: 31(1.3)$ & 157 \\
\hline $12^{h} 44^{m} 34.80^{s},-10 \operatorname{deg} 38^{\prime} 42.7^{\prime \prime}$ & Aug 18 at 19:36:02 (1.3) & 97 \\
\hline $12^{h} 51^{m} 4.80^{s},-14 \operatorname{deg} 42^{\prime} 55.4^{\prime \prime}$ & Aug 18 at 19:38:07 (1.3) & 115 \\
\hline $12^{h} 51^{m} 55.44^{s},-14 \operatorname{deg} 24^{\prime} 08.3^{\prime \prime}$ & Aug 18 at $19: 40: 43(1.3)$ & 122 \\
\hline $12^{h} 51^{m} 30.00^{s},-14 \operatorname{deg} 05^{\prime} 21.1^{\prime \prime}$ & Aug 18 at 19:43:13 (1.3) & 112 \\
\hline $12^{h} 50^{m} 12.48^{s},-14 \operatorname{deg} 05^{\prime} 21.1^{\prime \prime}$ & Aug 18 at $19: 45: 38(1.3)$ & 115 \\
\hline $12^{h} 48^{m} 55.20^{s},-14 \operatorname{deg} 05^{\prime} 21.1^{\prime \prime}$ & Aug 18 at 19:48:03 (1.3) & 117 \\
\hline $12^{h} 48^{m} 31.20^{s},-13 \operatorname{deg} 46^{\prime} 34.0^{\prime \prime}$ & Aug 18 at 19:50:28 (1.3) & 117 \\
\hline $12^{h} 49^{m} 48.72^{s},-13 \operatorname{deg} 46^{\prime} 34.0^{\prime \prime}$ & Aug 18 at $19: 52: 53(1.3)$ & 120 \\
\hline $12^{h} 51^{m} 6.00^{s},-13 \operatorname{deg} 46^{\prime} 34.0^{\prime \prime}$ & Aug 18 at $19: 55: 18$ (1.3) & 117 \\
\hline $12^{h} 36^{m} 13.44^{s},-13 \operatorname{deg} 08^{\prime} 59.6^{\prime \prime}$ & Aug 18 at $21: 08: 57$ (1.4) & 27 \\
\hline $12^{h} 35^{m} 54.72^{s},-12 \operatorname{deg} 50^{\prime} 12.5^{\prime \prime}$ & Aug 18 at $21: 11: 42(1.4)$ & 120 \\
\hline $12^{h} 56^{m} 47.76^{s},-19 \operatorname{deg} 24^{\prime} 42.5^{\prime \prime}$ & Aug 18 at $21: 14: 07(1.4)$ & 115 \\
\hline $12^{h} 55^{m} 58.56^{s},-19 \operatorname{deg} 05^{\prime} 55.3^{\prime \prime}$ & Aug 18 at $21: 16: 48(1.4)$ & 120 \\
\hline $12^{h} 55^{m} 28.08^{s},-19 \operatorname{deg} 24^{\prime} 42.5^{\prime \prime}$ & Aug 18 at $21: 19: 18(1.4)$ & 117 \\
\hline
\end{tabular}

${ }^{a}$ Observations were 2017, times in parentheses are days since the GW trigger. 


\begin{tabular}{|c|c|c|}
\hline $\begin{array}{l}\text { Pointing direction } \\
\text { (J2000) }\end{array}$ & $\begin{array}{l}\text { Start time }^{a} \\
\text { (UTC) }\end{array}$ & $\begin{array}{c}\text { Exposure } \\
\text { (s) }\end{array}$ \\
\hline $12^{h} 56^{m} 18.48^{s},-19 \operatorname{deg} 43^{\prime} 29.6^{\prime \prime}$ & Aug 18 at $21: 21: 43(1.4)$ & 117 \\
\hline $12^{h} 54^{m} 58.80^{s},-19 \operatorname{deg} 43^{\prime} 29.6^{\prime \prime}$ & Aug 18 at $21: 24: 14$ (1.4) & 112 \\
\hline $12^{h} 54^{m} 30.72^{s},-20 \operatorname{deg} 02^{\prime} 16.8^{\prime \prime}$ & Aug 18 at $21: 26: 39$ (1.4) & 115 \\
\hline $12^{h} 54^{m} 4.08^{s},-20 \operatorname{deg} 21^{\prime} 03.6^{\prime \prime}$ & Aug 18 at 21:29:04 (1.4) & 120 \\
\hline $12^{h} 53^{m} 10.80^{s},-20 \operatorname{deg} 02^{\prime} 16.8^{\prime \prime}$ & Aug 18 at $21: 31: 29(1.4)$ & 122 \\
\hline $12^{h} 53^{m} 38.88^{s},-19 \operatorname{deg} 43^{\prime} 29.6^{\prime \prime}$ & Aug 18 at $21: 33: 58$ (1.4) & 112 \\
\hline $12^{h} 52^{m} 55.20^{s},-17 \operatorname{deg} 13^{\prime} 12.4^{\prime \prime}$ & Aug 18 at $22: 47: 52(1.4)$ & 107 \\
\hline $12^{h} 54^{m} 13.92^{s},-17 \operatorname{deg} 13^{\prime} 12.4^{\prime \prime}$ & Aug 18 at $22: 50: 17$ (1.4) & 117 \\
\hline $12^{h} 55^{m} 32.64^{s},-17 \operatorname{deg} 13^{\prime} 12.4^{\prime \prime}$ & Aug 18 at $22: 52: 41(1.4)$ & 117 \\
\hline $12^{h} 56^{m} 12.72^{s},-17 \operatorname{deg} 31^{\prime} 59.5^{\prime \prime}$ & Aug 18 at 22:55:07 (1.4) & 120 \\
\hline $12^{h} 55^{m} 35.52^{s},-17 \operatorname{deg} 50^{\prime} 46.7^{\prime \prime}$ & Aug 18 at $22: 57: 32(1.4)$ & 115 \\
\hline $12^{h} 54^{m} 16.56^{s},-17 \operatorname{deg} 50^{\prime} 46.7^{\prime \prime}$ & Aug 18 at $22: 59: 57$ (1.4) & 112 \\
\hline $12^{h} 54^{m} 59.76^{s},-18 \operatorname{deg} 09^{\prime} 33.8^{\prime \prime}$ & Aug 18 at 23:02:22 (1.4) & 120 \\
\hline $12^{h} 56^{m} 18.72^{s},-18 \operatorname{deg} 09^{\prime} 33.8^{\prime \prime}$ & Aug 18 at 23:04:47 (1.4) & 117 \\
\hline $12^{h} 57^{m} 37.92^{s},-18 \operatorname{deg} 09^{\prime} 33.8^{\prime \prime}$ & Aug 18 at 23:07:12 (1.4) & 117 \\
\hline $12^{h} 58^{m} 22.80^{s},-18 \operatorname{deg} 28^{\prime} 21.0^{\prime \prime}$ & Aug 18 at 23:09:37 (1.4) & 120 \\
\hline $12^{h} 59^{m} 9.12^{s},-18 \operatorname{deg} 47^{\prime} 08.2^{\prime \prime}$ & Aug 18 at $23: 12: 02(1.4)$ & 117 \\
\hline $12^{h} 50^{m} 15.12^{s},-15 \operatorname{deg} 01^{\prime} 42.6^{\prime \prime}$ & Aug 19 at $02: 13: 35$ (1.6) & 102 \\
\hline $12^{h} 51^{m} 33.12^{s},-15 \operatorname{deg} 01^{\prime} 42.6^{\prime \prime}$ & Aug 19 at $02: 15: 42(1.6)$ & 120 \\
\hline $12^{h} 52^{m} 22.32^{s},-14 \operatorname{deg} 42^{\prime} 55.4^{\prime \prime}$ & Aug 19 at $02: 18: 06(1.6)$ & 125 \\
\hline $12^{h} 53^{m} 40.08^{s},-14 \operatorname{deg} 42^{\prime} 55.4^{\prime \prime}$ & Aug 19 at $02: 20: 37$ (1.6) & 117 \\
\hline $12^{h} 54^{m} 57.84^{s},-14 \operatorname{deg} 42^{\prime} 55.4^{\prime \prime}$ & Aug 19 at $02: 23: 03$ (1.6) & 120 \\
\hline $12^{h} 55^{m} 26.40^{s},-15 \operatorname{deg} 01^{\prime} 42.6^{\prime \prime}$ & Aug 19 at $02: 25: 27$ (1.6) & 117 \\
\hline $12^{h} 56^{m} 44.16^{s},-15 \operatorname{deg} 01^{\prime} 42.6^{\prime \prime}$ & Aug 19 at $02: 27: 53$ (1.6) & 117 \\
\hline $12^{h} 58^{m} 2.16^{s},-15 \operatorname{deg} 01^{\prime} 42.6^{\prime \prime}$ & Aug 19 at $02: 30: 18$ (1.6) & 120 \\
\hline $12^{h} 58^{m} 32.40^{s},-15 \operatorname{deg} 20^{\prime} 29.8^{\prime \prime}$ & Aug 19 at $02: 32: 42(1.6)$ & 112 \\
\hline $13^{h} 16^{m} 20.64^{s},-26 \operatorname{deg} 36^{\prime} 46.4^{\prime \prime}$ & Aug 19 at $03: 35: 12$ (1.6) & 87 \\
\hline $13^{h} 14^{m} 56.64^{s},-26 \operatorname{deg} 36^{\prime} 46.4^{\prime \prime}$ & Aug 19 at $03: 37: 31$ (1.6) & 110 \\
\hline $13^{h} 13^{m} 32.40^{s},-26 \operatorname{deg} 36^{\prime} 46.4^{\prime \prime}$ & Aug 19 at $03: 39: 56(1.6)$ & 112 \\
\hline $13^{h} 15^{m} 1.68^{s},-26 \operatorname{deg} 55^{\prime} 33.6^{\prime \prime}$ & Aug 19 at $03: 42: 20(1.6)$ & 125 \\
\hline $13^{h} 16^{m} 26.16^{s},-26 \operatorname{deg} 55^{\prime} 33.6^{\prime \prime}$ & Aug 19 at $03: 44: 51$ (1.6) & 117 \\
\hline $13^{h} 17^{m} 50.40^{s},-26 \operatorname{deg} 55^{\prime} 33.6^{\prime \prime}$ & Aug 19 at $03: 47: 16$ (1.6) & 117 \\
\hline $13^{h} 19^{m} 22.08^{s},-27 \operatorname{deg} 14^{\prime} 20.8^{\prime \prime}$ & Aug 19 at $03: 49: 41$ (1.6) & 122 \\
\hline $13^{h} 20^{m} 46.56^{s},-27 \operatorname{deg} 14^{\prime} 20.8^{\prime \prime}$ & Aug 19 at $03: 52: 12(1.6)$ & 117 \\
\hline $13^{h} 19^{m} 14.64^{s},-26 \operatorname{deg} 55^{\prime} 33.6^{\prime \prime}$ & Aug 19 at 03:54:37 (1.6) & 122 \\
\hline $13^{h} 17^{m} 44.64^{s},-26 \operatorname{deg} 36^{\prime} 46.4^{\prime \prime}$ & Aug 19 at 03:57:07 (1.6) & 122 \\
\hline
\end{tabular}

${ }^{a}$ Observations were 2017, times in parentheses are days since the GW trigger. 


\begin{tabular}{|c|c|c|}
\hline $\begin{array}{l}\text { Pointing direction } \\
\text { (J2000) }\end{array}$ & $\begin{array}{l}\text { Start time }^{a} \\
\text { (UTC) }\end{array}$ & $\begin{array}{c}\text { Exposure } \\
\text { (s) }\end{array}$ \\
\hline $13^{h} 16^{m} 16.56^{s},-26 \operatorname{deg} 17^{\prime} 59.3^{\prime \prime}$ & Aug 19 at $03: 59: 38$ (1.6) & 125 \\
\hline $13^{h} 14^{m} 50.40^{s},-25 \operatorname{deg} 59^{\prime} 12.1^{\prime \prime}$ & Aug 19 at 04:02:09 (1.6) & 122 \\
\hline $13^{h} 13^{m} 26.88^{s},-25 \operatorname{deg} 59^{\prime} 12.1^{\prime \prime}$ & Aug 19 at 04:04:39 (1.6) & 120 \\
\hline $13^{h} 12^{m} 3.12^{s},-25 \operatorname{deg} 59^{\prime} 12.1^{\prime \prime}$ & Aug 19 at 04:07:04 (1.6) & 115 \\
\hline $13^{h} 13^{m} 26.16^{s},-25 \operatorname{deg} 40^{\prime} 25.0^{\prime \prime}$ & Aug 19 at 04:09:29 (1.6) & 127 \\
\hline $13^{h} 28^{m} 42.00^{s},-31 \operatorname{deg} 56^{\prime} 07.8^{\prime \prime}$ & Aug 19 at $04: 12: 00$ (1.6) & 117 \\
\hline $13^{h} 58^{m} 16.32^{s},-48 \operatorname{deg} 12^{\prime} 58.7^{\prime \prime}$ & Aug 19 at $04: 14: 39$ (1.6) & 117 \\
\hline $12^{h} 54^{m} 5.04^{s},-14 \operatorname{deg} 05^{\prime} 21.1^{\prime \prime}$ & Aug 19 at 05:06:57 (1.7) & 80 \\
\hline $12^{h} 53^{m} 13.20^{s},-14 \operatorname{deg} 24^{\prime} 08.3^{\prime \prime}$ & Aug 19 at $05: 10: 42(1.7)$ & 117 \\
\hline $12^{h} 52^{m} 47.52^{s},-14 \operatorname{deg} 05^{\prime} 21.1^{\prime \prime}$ & Aug 19 at $05: 13: 12(1.7)$ & 120 \\
\hline $12^{h} 53^{m} 40.80^{s},-13 \operatorname{deg} 46^{\prime} 34.0^{\prime \prime}$ & Aug 19 at $05: 15: 37$ (1.7) & 120 \\
\hline $12^{h} 54^{m} 35.28^{s},-13 \operatorname{deg} 27^{\prime} 46.8^{\prime \prime}$ & Aug 19 at $05: 18: 08$ (1.7) & 122 \\
\hline $12^{h} 55^{m} 52.56^{s},-13 \operatorname{deg} 27^{\prime} 46.8^{\prime \prime}$ & Aug 19 at $05: 20: 37(1.7)$ & 115 \\
\hline $12^{h} 54^{m} 58.08^{s},-13 \operatorname{deg} 46^{\prime} 34.0^{\prime \prime}$ & Aug 19 at $05: 23: 03$ (1.7) & 122 \\
\hline $12^{h} 55^{m} 22.56^{s},-14 \operatorname{deg} 05^{\prime} 21.1^{\prime \prime}$ & Aug 19 at $05: 25: 34$ (1.7) & 112 \\
\hline $12^{h} 55^{m} 48.24^{s},-14 \operatorname{deg} 24^{\prime} 08.3^{\prime \prime}$ & Aug 19 at $05: 27: 59$ (1.7) & 112 \\
\hline $12^{h} 54^{m} 30.72^{s},-14 \operatorname{deg} 24^{\prime} 08.3^{\prime \prime}$ & Aug 19 at $05: 30: 24(1.7)$ & 117 \\
\hline $13^{h} 15^{m} 50.16^{s},-28 \operatorname{deg} 29^{\prime} 29.4^{\prime \prime}$ & Aug 19 at $05: 32: 49(1.7)$ & 115 \\
\hline $13^{h} 15^{m} 37.44^{s},-28 \operatorname{deg} 10^{\prime} 42.2^{\prime \prime}$ & Aug 19 at $05: 35: 38$ (1.7) & 115 \\
\hline $13^{h} 17^{m} 2.64^{s},-28 \operatorname{deg} 10^{\prime} 42.2^{\prime \prime}$ & Aug 19 at $05: 38: 03$ (1.7) & 117 \\
\hline $13^{h} 17^{m} 15.60^{s},-28 \operatorname{deg} 29^{\prime} 29.4^{\prime \prime}$ & Aug 19 at $05: 40: 28$ (1.7) & 115 \\
\hline $13^{h} 16^{m} 4.32^{s},-28 \operatorname{deg} 48^{\prime} 16.6^{\prime \prime}$ & Aug 19 at $05: 42: 53(1.7)$ & 120 \\
\hline $13^{h} 16^{m} 19.92^{s},-29 \operatorname{deg} 07^{\prime} 03.4^{\prime \prime}$ & Aug 19 at $05: 45: 24(1.7)$ & 112 \\
\hline $13^{h} 29^{m} 23.28^{s},-34 \operatorname{deg} 26^{\prime} 24.7^{\prime \prime}$ & Aug 19 at $05: 47: 49(1.7)$ & 115 \\
\hline $13^{h} 58^{m} 32.88^{s},-43 \operatorname{deg} 49^{\prime} 58.8^{\prime \prime}$ & Aug 19 at 05:50:27 (1.7) & 117 \\
\hline $14^{h} 20^{m} 19.92^{s},-55 \operatorname{deg} 06^{\prime} 15.8^{\prime \prime}$ & Aug 19 at $05: 53: 11(1.7)$ & 117 \\
\hline $12^{h} 54^{m} 53.76^{s},-16 \operatorname{deg} 54^{\prime} 25.2^{\prime \prime}$ & Aug 19 at $06: 47: 14(1.8)$ & 87 \\
\hline $12^{h} 55^{m} 35.04^{s},-16 \operatorname{deg} 35^{\prime} 38.0^{\prime \prime}$ & Aug 19 at $06: 49: 07$ (1.8) & 117 \\
\hline $12^{h} 56^{m} 53.52^{s},-16 \operatorname{deg} 35^{\prime} 38.0^{\prime \prime}$ & Aug 19 at $06: 51: 32(1.8)$ & 120 \\
\hline $12^{h} 56^{m} 17.52^{s},-16 \operatorname{deg} 16^{\prime} 50.9^{\prime \prime}$ & Aug 19 at $06: 53: 57(1.8)$ & 120 \\
\hline $12^{h} 55^{m} 42.96^{s},-15 \operatorname{deg} 58^{\prime} 03.7^{\prime \prime}$ & Aug 19 at $06: 56: 22(1.8)$ & 120 \\
\hline $12^{h} 56^{m} 28.08^{s},-15 \operatorname{deg} 39^{\prime} 16.6^{\prime \prime}$ & Aug 19 at $06: 58: 47(1.8)$ & 120 \\
\hline $12^{h} 57^{m} 46.08^{s},-15 \operatorname{deg} 39^{\prime} 16.6^{\prime \prime}$ & Aug 19 at $07: 01: 12(1.8)$ & 120 \\
\hline $12^{h} 59^{m} 4.08^{s},-15 \operatorname{deg} 39^{\prime} 16.6^{\prime \prime}$ & Aug 19 at 07:03:37 (1.8) & 120 \\
\hline $12^{h} 59^{m} 50.40^{s},-15 \operatorname{deg} 20^{\prime} 29.8^{\prime \prime}$ & Aug 19 at 07:06:02 (1.8) & 110 \\
\hline $13^{h} 01^{m} 8.16^{s},-15 \operatorname{deg} 20^{\prime} 29.8^{\prime \prime}$ & Aug 19 at 07:08:27 (1.8) & 120 \\
\hline
\end{tabular}

${ }^{a}$ Observations were 2017, times in parentheses are days since the GW trigger. 


\begin{tabular}{|c|c|c|}
\hline $\begin{array}{l}\text { Pointing direction } \\
\text { (J2000) }\end{array}$ & $\begin{array}{l}\text { Start time }^{a} \\
\text { (UTC) }\end{array}$ & $\begin{array}{c}\text { Exposure } \\
\text { (s) }\end{array}$ \\
\hline $13^{h} 00^{m} 37.68^{s},-15 \operatorname{deg} 01^{\prime} 42.6^{\prime \prime}$ & Aug 19 at $07: 10: 52(1.8)$ & 115 \\
\hline $12^{h} 59^{m} 19.92^{s},-15 \operatorname{deg} 01^{\prime} 42.6^{\prime \prime}$ & Aug 19 at $07: 13: 17(1.8)$ & 112 \\
\hline $12^{h} 58^{m} 50.88^{s},-14 \operatorname{deg} 42^{\prime} 55.4^{\prime \prime}$ & Aug 19 at $07: 15: 42(1.8)$ & 117 \\
\hline $13^{h} 00^{m} 8.64^{s},-14 \operatorname{deg} 42^{\prime} 55.4^{\prime \prime}$ & Aug 19 at 07:18:07 (1.8) & 120 \\
\hline $13^{h} 08^{m} 7.44^{s},-24 \operatorname{deg} 25^{\prime} 16.7^{\prime \prime}$ & Aug 19 at $07: 20: 31(1.8)$ & 117 \\
\hline $13^{h} 36^{m} 12.72^{s},-34 \operatorname{deg} 07^{\prime} 37.6^{\prime \prime}$ & Aug 19 at $07: 23: 15(1.8)$ & 117 \\
\hline $14^{h} 09^{m} 16.08^{s},-49 \operatorname{deg} 09^{\prime} 20.2^{\prime \prime}$ & Aug 19 at $07: 26: 00(1.8)$ & 117 \\
\hline $14^{h} 15^{m} 53.04^{s},-51 \operatorname{deg} 58^{\prime} 24.2^{\prime \prime}$ & Aug 19 at $07: 28: 51(1.8)$ & 117 \\
\hline $12^{h} 48^{m} 9.12^{s},-15 \operatorname{deg} 20^{\prime} 29.8^{\prime \prime}$ & Aug 19 at $08: 22: 14(1.8)$ & 77 \\
\hline $12^{h} 49^{m} 26.88^{s},-15 \operatorname{deg} 20^{\prime} 29.8^{\prime \prime}$ & Aug 19 at $08: 24: 07$ (1.8) & 117 \\
\hline $12^{h} 48^{m} 39.84^{s},-15 \operatorname{deg} 39^{\prime} 16.6^{\prime \prime}$ & Aug 19 at $08: 26: 32(1.8)$ & 115 \\
\hline $12^{h} 49^{m} 12.24^{s},-15 \operatorname{deg} 58^{\prime} 03.7^{\prime \prime}$ & Aug 19 at $08: 28: 57$ (1.8) & 120 \\
\hline $12^{h} 50^{m} 30.48^{s},-15 \operatorname{deg} 58^{\prime} 03.7^{\prime \prime}$ & Aug 19 at $08: 31: 21(1.8)$ & 117 \\
\hline $12^{h} 49^{m} 46.08^{s},-16 \operatorname{deg} 16^{\prime} 50.9^{\prime \prime}$ & Aug 19 at $08: 33: 47(1.8)$ & 120 \\
\hline $12^{h} 50^{m} 21.36^{s},-16 \operatorname{deg} 35^{\prime} 38.0^{\prime \prime}$ & Aug 19 at $08: 36: 12(1.8)$ & 117 \\
\hline $12^{h} 51^{m} 39.84^{s},-16 \operatorname{deg} 35^{\prime} 38.0^{\prime \prime}$ & Aug 19 at $08: 38: 37$ (1.8) & 120 \\
\hline $12^{h} 52^{m} 16.80^{s},-16 \operatorname{deg} 54^{\prime} 25.2^{\prime \prime}$ & Aug 19 at $08: 41: 02(1.8)$ & 117 \\
\hline $12^{h} 51^{m} 36.48^{s},-17 \operatorname{deg} 13^{\prime} 12.4^{\prime \prime}$ & Aug 19 at $08: 43: 27(1.8)$ & 112 \\
\hline $12^{h} 52^{m} 16.32^{s},-17 \operatorname{deg} 31^{\prime} 59.5^{\prime \prime}$ & Aug 19 at $08: 45: 52(1.8)$ & 120 \\
\hline $12^{h} 53^{m} 35.04^{s},-17 \operatorname{deg} 31^{\prime} 59.5^{\prime \prime}$ & Aug 19 at $08: 48: 16(1.8)$ & 117 \\
\hline $12^{h} 54^{m} 54.00^{s},-17 \operatorname{deg} 31^{\prime} 59.5^{\prime \prime}$ & Aug 19 at $08: 50: 42(1.8)$ & 117 \\
\hline $13^{h} 02^{m} 10.32^{s},-17 \operatorname{deg} 50^{\prime} 46.7^{\prime \prime}$ & Aug 19 at $08: 53: 06(1.8)$ & 122 \\
\hline $13^{h} 02^{m} 46.80^{s},-17 \operatorname{deg} 31^{\prime} 59.5^{\prime \prime}$ & Aug 19 at $08: 55: 39(1.8)$ & 112 \\
\hline $13^{h} 30^{m} 9.60^{s},-34 \operatorname{deg} 07^{\prime} 37.6^{\prime \prime}$ & Aug 19 at 08:58:04 (1.8) & 117 \\
\hline $14^{h} 11^{m} 49.20^{s},-51 \operatorname{deg} 58^{\prime} 24.2^{\prime \prime}$ & Aug 19 at $09: 03: 36(1.8)$ & 152 \\
\hline $12^{h} 54^{m} 8.64^{s},-15 \operatorname{deg} 01^{\prime} 42.6^{\prime \prime}$ & Aug 19 at 09:58:13 (1.9) & 85 \\
\hline $12^{h} 54^{m} 38.64^{s},-15 \operatorname{deg} 20^{\prime} 29.8^{\prime \prime}$ & Aug 19 at 10:00:07 (1.9) & 117 \\
\hline $12^{h} 55^{m} 10.08^{s},-15 \operatorname{deg} 39^{\prime} 16.6^{\prime \prime}$ & Aug 19 at 10:02:32 (1.9) & 115 \\
\hline $12^{h} 55^{m} 56.64^{s},-15 \operatorname{deg} 20^{\prime} 29.8^{\prime \prime}$ & Aug 19 at 10:04:57 (1.9) & 110 \\
\hline $12^{h} 57^{m} 14.40^{s},-15 \operatorname{deg} 20^{\prime} 29.8^{\prime \prime}$ & Aug 19 at 10:07:22 (1.9) & 110 \\
\hline $12^{h} 58^{m} 36.24^{s},-20 \operatorname{deg} 58^{\prime} 37.9^{\prime \prime}$ & Aug 19 at 10:09:47 (1.9) & 117 \\
\hline $12^{h} 59^{m} 56.88^{s},-20 \operatorname{deg} 58^{\prime} 37.9^{\prime \prime}$ & Aug 19 at $10: 12: 24$ (1.9) & 120 \\
\hline $12^{h} 59^{m} 34.80^{s},-21 \operatorname{deg} 17^{\prime} 25.1^{\prime \prime}$ & Aug 19 at $10: 14: 48$ (1.9) & 120 \\
\hline $12^{h} 58^{m} 14.16^{s},-21 \operatorname{deg} 17^{\prime} 25.1^{\prime \prime}$ & Aug 19 at $10: 17: 14(1.9)$ & 120 \\
\hline $12^{h} 57^{m} 15.84^{s},-20 \operatorname{deg} 58^{\prime} 37.9^{\prime \prime}$ & Aug 19 at 10:19:39 (1.9) & 127 \\
\hline $12^{h} 57^{m} 39.60^{s},-20 \operatorname{deg} 39^{\prime} 50.8^{\prime \prime}$ & Aug 19 at 10:22:10 (1.9) & 120 \\
\hline
\end{tabular}

${ }^{a}$ Observations were 2017, times in parentheses are days since the GW trigger. 


\begin{tabular}{|c|c|c|}
\hline $\begin{array}{l}\text { Pointing direction } \\
\text { (J2000) }\end{array}$ & $\begin{array}{l}\text { Start time }^{a} \\
\text { (UTC) }\end{array}$ & $\begin{array}{c}\text { Exposure } \\
\text { (s) }\end{array}$ \\
\hline $12^{h} 56^{m} 44.40^{s},-20 \operatorname{deg} 21^{\prime} 03.6^{\prime \prime}$ & Aug 19 at 10:24:35 (1.9) & 125 \\
\hline $12^{h} 56^{m} 19.20^{s},-20 \operatorname{deg} 39^{\prime} 50.8^{\prime \prime}$ & Aug 19 at 10:27:05 (1.9) & 115 \\
\hline $12^{h} 55^{m} 55.44^{s},-20 \operatorname{deg} 58^{\prime} 37.9^{\prime \prime}$ & Aug 19 at 10:29:30 (1.9) & 115 \\
\hline $13^{h} 04^{m} 1.20^{s},-22 \operatorname{deg} 13^{\prime} 46.6^{\prime \prime}$ & Aug 19 at 10:31:55 (1.9) & 122 \\
\hline $13^{h} 31^{m} 45.60^{s},-33 \operatorname{deg} 30^{\prime} 03.2^{\prime \prime}$ & Aug 19 at 10:34:28 (1.9) & 115 \\
\hline $14^{h} 08^{m} 10.32^{s},-48 \operatorname{deg} 31^{\prime} 45.8^{\prime \prime}$ & Aug 19 at $10: 37: 15$ (1.9) & 117 \\
\hline $14^{h} 17^{m} 12.72^{s},-55 \operatorname{deg} 25^{\prime} 03.0^{\prime \prime}$ & Aug 19 at $10: 40: 06$ (1.9) & 127 \\
\hline $12^{h} 48^{m} 27.36^{s},-12 \operatorname{deg} 31^{\prime} 25.3^{\prime \prime}$ & Aug 19 at $11: 33: 02(2.0)$ & 97 \\
\hline $12^{h} 48^{m} 10.56^{s},-12 \operatorname{deg} 12^{\prime} 38.2^{\prime \prime}$ & Aug 19 at 11:35:07 (2.0) & 117 \\
\hline $12^{h} 49^{m} 44.40^{s},-12 \operatorname{deg} 31^{\prime} 25.3^{\prime \prime}$ & Aug 19 at $11: 37: 31(2.0)$ & 120 \\
\hline $12^{h} 51^{m} 19.68^{s},-12 \operatorname{deg} 50^{\prime} 12.5^{\prime \prime}$ & Aug 19 at $11: 40: 03$ (2.0) & 122 \\
\hline $12^{h} 52^{m} 18.24^{s},-12 \operatorname{deg} 31^{\prime} 25.3^{\prime \prime}$ & Aug 19 at $11: 42: 33$ (2.0) & 122 \\
\hline $12^{h} 53^{m} 35.28^{s},-12 \operatorname{deg} 31^{\prime} 25.3^{\prime \prime}$ & Aug 19 at $11: 45: 04(2.0)$ & 120 \\
\hline $12^{h} 54^{m} 52.08^{s},-12 \operatorname{deg} 31^{\prime} 25.3^{\prime \prime}$ & Aug 19 at $11: 47: 29(2.0)$ & 115 \\
\hline $12^{h} 53^{m} 18.00^{s},-12 \operatorname{deg} 12^{\prime} 38.2^{\prime \prime}$ & Aug 19 at $11: 49: 54(2.0)$ & 117 \\
\hline $12^{h} 52^{m} 1.20^{s},-12 \operatorname{deg} 12^{\prime} 38.2^{\prime \prime}$ & Aug 19 at $11: 52: 24(2.0)$ & 117 \\
\hline $12^{h} 53^{m} 2.40^{s},-11 \operatorname{deg} 53^{\prime} 51.0^{\prime \prime}$ & Aug 19 at $11: 54: 49(2.0)$ & 120 \\
\hline $12^{h} 54^{m} 35.04^{s},-12 \operatorname{deg} 12^{\prime} 38.2^{\prime \prime}$ & Aug 19 at $11: 57: 20(2.0)$ & 122 \\
\hline $12^{h} 54^{m} 19.20^{s},-11 \operatorname{deg} 53^{\prime} 51.0^{\prime \prime}$ & Aug 19 at $11: 59: 51(2.0)$ & 120 \\
\hline $12^{h} 52^{m} 48.00^{s},-11 \operatorname{deg} 35^{\prime} 03.8^{\prime \prime}$ & Aug 19 at $12: 02: 15(2.0)$ & 125 \\
\hline $13^{h} 10^{m} 52.32^{s},-24 \operatorname{deg} 25^{\prime} 16.7^{\prime \prime}$ & Aug 19 at $12: 04: 46(2.0)$ & 112 \\
\hline $13^{h} 09^{m} 24.24^{s},-24 \operatorname{deg} 44^{\prime} 03.5^{\prime \prime}$ & Aug 19 at 12:07:34 (2.0) & 122 \\
\hline $13^{h} 36^{m} 58.80^{s},-33 \operatorname{deg} 48^{\prime} 50.4^{\prime \prime}$ & Aug 19 at $12: 10: 04(2.0)$ & 117 \\
\hline $13^{h} 55^{m} 7.68^{s},-44 \operatorname{deg} 08^{\prime} 46.0^{\prime \prime}$ & Aug 19 at $12: 12: 49(2.0)$ & 115 \\
\hline $14^{h} 17^{m} 45.60^{s},-52 \operatorname{deg} 35^{\prime} 58.6^{\prime \prime}$ & Aug 19 at $12: 15: 33(2.0)$ & 117 \\
\hline $12^{h} 47^{m} 21.84^{s},-15 \operatorname{deg} 39^{\prime} 16.6^{\prime \prime}$ & Aug 19 at 13:08:56 (2.0) & 102 \\
\hline $12^{h} 47^{m} 54.00^{s},-15 \operatorname{deg} 58^{\prime} 03.7^{\prime \prime}$ & Aug 19 at $13: 11: 06(2.0)$ & 117 \\
\hline $12^{h} 48^{m} 27.84^{s},-16 \operatorname{deg} 16^{\prime} 50.9^{\prime \prime}$ & Aug 19 at $13: 13: 32(2.0)$ & 120 \\
\hline $12^{h} 49^{m} 2.88^{s},-16 \operatorname{deg} 35^{\prime} 38.0^{\prime \prime}$ & Aug 19 at $13: 15: 57$ (2.0) & 120 \\
\hline $12^{h} 49^{m} 39.60^{s},-16 \operatorname{deg} 54^{\prime} 25.2^{\prime \prime}$ & Aug 19 at $13: 18: 22(2.0)$ & 120 \\
\hline $12^{h} 50^{m} 58.08^{s},-16 \operatorname{deg} 54^{\prime} 25.2^{\prime \prime}$ & Aug 19 at $13: 20: 47$ (2.0) & 117 \\
\hline $12^{h} 50^{m} 17.76^{s},-17 \operatorname{deg} 13^{\prime} 12.4^{\prime \prime}$ & Aug 19 at $13: 23: 12(2.0)$ & 22 \\
\hline $13^{h} 44^{m} 5.28^{s},-41 \operatorname{deg} 00^{\prime} 54.7^{\prime \prime}$ & Aug 19 at $13: 48: 23(2.0)$ & 87 \\
\hline $14^{h} 08^{m} 8.88^{s},-51 \operatorname{deg} 20^{\prime} 49.9^{\prime \prime}$ & Aug 19 at $13: 50: 34(2.0)$ & 155 \\
\hline $13^{h} 31^{m} 40.80^{s},-34 \operatorname{deg} 45^{\prime} 11.9^{\prime \prime}$ & Aug 19 at $15: 23: 16(2.1)$ & 35 \\
\hline $14^{h} 07^{m} 40.32^{s},-48 \operatorname{deg} 12^{\prime} 58.7^{\prime \prime}$ & Aug 19 at $15: 24: 41$ (2.1) & 117 \\
\hline
\end{tabular}

${ }^{a}$ Observations were 2017, times in parentheses are days since the GW trigger. 


\begin{tabular}{|c|c|c|}
\hline $\begin{array}{l}\text { Pointing direction } \\
\text { (J2000) }\end{array}$ & $\begin{array}{l}\text { Start time }^{a} \\
\text { (UTC) }\end{array}$ & $\begin{array}{c}\text { Exposure } \\
\text { (s) }\end{array}$ \\
\hline $14^{h} 18^{m} 30.72^{s},-55 \operatorname{deg} 43^{\prime} 50.2^{\prime \prime}$ & Aug 19 at $15: 27: 30(2.1)$ & 117 \\
\hline $13^{h} 02^{m} 24.00^{s},-22 \operatorname{deg} 32^{\prime} 33.7^{\prime \prime}$ & Aug 19 at $16: 37: 27$ (2.2) & 112 \\
\hline $13^{h} 03^{m} 30.48^{s},-22 \operatorname{deg} 51^{\prime} 20.9^{\prime \prime}$ & Aug 19 at $16: 39: 53(2.2)$ & 117 \\
\hline $13^{h} 02^{m} 9.12^{s},-22 \operatorname{deg} 51^{\prime} 20.9^{\prime \prime}$ & Aug 19 at $16: 42: 24$ (2.2) & 117 \\
\hline $13^{h} 01^{m} 2.64^{s},-22 \operatorname{deg} 32^{\prime} 33.7^{\prime \prime}$ & Aug 19 at $16: 44: 49(2.2)$ & 122 \\
\hline $12^{h} 59^{m} 41.28^{s},-22 \operatorname{deg} 32^{\prime} 33.7^{\prime \prime}$ & Aug 19 at $16: 47: 19(2.2)$ & 117 \\
\hline $12^{h} 58^{m} 4.32^{s},-22 \operatorname{deg} 51^{\prime} 20.9^{\prime \prime}$ & Aug 19 at $16: 49: 43$ (2.2) & 122 \\
\hline $12^{h} 58^{m} 19.92^{s},-22 \operatorname{deg} 32^{\prime} 33.7^{\prime \prime}$ & Aug 19 at $16: 52: 15(2.2)$ & 112 \\
\hline $13^{h} 24^{m} 30.00^{s},-30 \operatorname{deg} 03^{\prime} 24.8^{\prime \prime}$ & Aug 19 at $16: 54: 40(2.2)$ & 115 \\
\hline $13^{h} 34^{m} 51.60^{s},-35 \operatorname{deg} 22^{\prime} 46.2^{\prime \prime}$ & Aug 19 at $16: 57: 22(2.2)$ & 117 \\
\hline $13^{h} 51^{m} 36.24^{s},-43 \operatorname{deg} 49^{\prime} 58.8^{\prime \prime}$ & Aug 19 at 17:00:00 (2.2) & 117 \\
\hline $14^{h} 16^{m} 39.12^{s},-53 \operatorname{deg} 32^{\prime} 20.0^{\prime \prime}$ & Aug 19 at $17: 02: 42(2.2)$ & 117 \\
\hline $12^{h} 47^{m} 9.60^{s},-16 \operatorname{deg} 16^{\prime} 50.9^{\prime \prime}$ & Aug 19 at $17: 56: 09(2.2)$ & 87 \\
\hline $12^{h} 47^{m} 44.64^{s},-16 \operatorname{deg} 35^{\prime} 38.0^{\prime \prime}$ & Aug 19 at 17:58:07 (2.2) & 112 \\
\hline $12^{h} 48^{m} 21.12^{s},-16 \operatorname{deg} 54^{\prime} 25.2^{\prime \prime}$ & Aug 19 at 18:00:31 (2.2) & 112 \\
\hline $12^{h} 48^{m} 59.28^{s},-17 \operatorname{deg} 13^{\prime} 12.4^{\prime \prime}$ & Aug 19 at 18:02:57 (2.2) & 115 \\
\hline $12^{h} 49^{m} 38.64^{s},-17 \operatorname{deg} 31^{\prime} 59.5^{\prime \prime}$ & Aug 19 at 18:05:22 (2.2) & 120 \\
\hline $12^{h} 50^{m} 19.92^{s},-17 \operatorname{deg} 50^{\prime} 46.7^{\prime \prime}$ & Aug 19 at 18:07:47 (2.2) & 120 \\
\hline $12^{h} 51^{m} 2.40^{s},-18 \operatorname{deg} 09^{\prime} 33.8^{\prime \prime}$ & Aug 19 at $18: 10: 12(2.2)$ & 117 \\
\hline $12^{h} 52^{m} 21.60^{s},-18 \operatorname{deg} 09^{\prime} 33.8^{\prime \prime}$ & Aug 19 at $18: 12: 37$ (2.2) & 120 \\
\hline $12^{h} 53^{m} 5.76^{s},-18 \operatorname{deg} 28^{\prime} 21.0^{\prime \prime}$ & Aug 19 at $18: 15: 02(2.2)$ & 117 \\
\hline $12^{h} 53^{m} 51.60^{s},-18 \operatorname{deg} 47^{\prime} 08.2^{\prime \prime}$ & Aug 19 at 18:17:27 (2.2) & 110 \\
\hline $12^{h} 54^{m} 39.12^{s},-19 \operatorname{deg} 05^{\prime} 55.3^{\prime \prime}$ & Aug 19 at $18: 19: 52(2.2)$ & 115 \\
\hline $12^{h} 54^{m} 8.40^{s},-19 \operatorname{deg} 24^{\prime} 42.5^{\prime \prime}$ & Aug 19 at $18: 22: 17$ (2.2) & 117 \\
\hline $12^{h} 53^{m} 19.44^{s},-19 \operatorname{deg} 05^{\prime} 55.3^{\prime \prime}$ & Aug 19 at $18: 24: 42(2.2)$ & 122 \\
\hline $12^{h} 52^{m} 32.40^{s},-18 \operatorname{deg} 47^{\prime} 08.2^{\prime \prime}$ & Aug 19 at $18: 27: 12(2.2)$ & 117 \\
\hline $13^{h} 07^{m} 59.76^{s},-20 \operatorname{deg} 58^{\prime} 37.9^{\prime \prime}$ & Aug 19 at $18: 29: 37$ (2.2) & 37 \\
\hline $13^{h} 45^{m} 52.80^{s},-39 \operatorname{deg} 08^{\prime} 11.8^{\prime \prime}$ & Aug 19 at $18: 35: 24(2.2)$ & 95 \\
\hline $14^{h} 06^{m} 6.96^{s},-53 \operatorname{deg} 32^{\prime} 20.0^{\prime \prime}$ & Aug 19 at $18: 37: 39(2.2)$ & 145 \\
\hline $13^{h} 01^{m} 28.08^{s},-17 \operatorname{deg} 31^{\prime} 59.5^{\prime \prime}$ & Aug 20 at $00: 18: 50(2.5)$ & 105 \\
\hline $13^{h} 00^{m} 47.28^{s},-17 \operatorname{deg} 13^{\prime} 12.4^{\prime \prime}$ & Aug 20 at $00: 21: 19(2.5)$ & 115 \\
\hline $13^{h} 00^{m} 7.92^{s},-16 \operatorname{deg} 54^{\prime} 25.2^{\prime \prime}$ & Aug 20 at $00: 23: 44(2.5)$ & 117 \\
\hline $12^{h} 59^{m} 28.56^{s},-17 \operatorname{deg} 13^{\prime} 12.4^{\prime \prime}$ & Aug 20 at $00: 26: 09(2.5)$ & 117 \\
\hline $13^{h} 00^{m} 9.12^{s},-17 \operatorname{deg} 31^{\prime} 59.5^{\prime \prime}$ & Aug 20 at $00: 28: 34(2.5)$ & 117 \\
\hline $12^{h} 59^{m} 32.40^{s},-17 \operatorname{deg} 50^{\prime} 46.7^{\prime \prime}$ & Aug 20 at $00: 30: 59(2.5)$ & 117 \\
\hline $13^{h} 00^{m} 51.36^{s},-17 \operatorname{deg} 50^{\prime} 46.7^{\prime \prime}$ & Aug 20 at $00: 33: 23$ (2.5) & 115 \\
\hline
\end{tabular}

${ }^{a}$ Observations were 2017, times in parentheses are days since the GW trigger. 


\begin{tabular}{|c|c|c|}
\hline $\begin{array}{l}\text { Pointing direction } \\
\text { (J2000) }\end{array}$ & $\begin{array}{l}\text { Start time }^{a} \\
\text { (UTC) }\end{array}$ & $\begin{array}{c}\text { Exposure } \\
\text { (s) }\end{array}$ \\
\hline $13^{h} 18^{m} 6.24^{s},-27 \operatorname{deg} 33^{\prime} 07.9^{\prime \prime}$ & Aug 20 at $00: 35: 49(2.5)$ & 117 \\
\hline $13^{h} 18^{m} 16.32^{s},-27 \operatorname{deg} 51^{\prime} 55.1^{\prime \prime}$ & Aug 20 at $00: 38: 32(2.5)$ & 112 \\
\hline $13^{h} 19^{m} 41.28^{s},-27 \operatorname{deg} 51^{\prime} 55.1^{\prime \prime}$ & Aug 20 at $00: 40: 56(2.5)$ & 117 \\
\hline $13^{h} 18^{m} 27.84^{s},-28 \operatorname{deg} 10^{\prime} 42.2^{\prime \prime}$ & Aug 20 at $00: 43: 22(2.5)$ & 125 \\
\hline $13^{h} 16^{m} 51.36^{s},-27 \operatorname{deg} 51^{\prime} 55.1^{\prime \prime}$ & Aug 20 at $00: 45: 53(2.5)$ & 125 \\
\hline $12^{h} 43^{m} 27.84^{s},-15 \operatorname{deg} 39^{\prime} 16.6^{\prime \prime}$ & Aug 20 at $01: 54: 17$ (2.6) & 72 \\
\hline $12^{h} 43^{m} 59.52^{s},-15 \operatorname{deg} 58^{\prime} 03.7^{\prime \prime}$ & Aug 20 at $01: 56: 07$ (2.6) & 107 \\
\hline $12^{h} 44^{m} 32.88^{s},-16 \operatorname{deg} 16^{\prime} 50.9^{\prime \prime}$ & Aug 20 at $01: 58: 32$ (2.6) & 117 \\
\hline $12^{h} 45^{m} 7.68^{s},-16 \operatorname{deg} 35^{\prime} 38.0^{\prime \prime}$ & Aug 20 at 02:00:57 (2.6) & 115 \\
\hline $12^{h} 45^{m} 44.16^{s},-16 \operatorname{deg} 54^{\prime} 25.2^{\prime \prime}$ & Aug 20 at $02: 03: 22$ (2.6) & 120 \\
\hline $12^{h} 46^{m} 21.84^{s},-17 \operatorname{deg} 13^{\prime} 12.4^{\prime \prime}$ & Aug 20 at $02: 05: 46(2.6)$ & 117 \\
\hline $12^{h} 47^{m} 1.20^{s},-17 \operatorname{deg} 31^{\prime} 59.5^{\prime \prime}$ & Aug 20 at $02: 08: 11$ (2.6) & 115 \\
\hline $12^{h} 47^{m} 42.00^{s},-17 \operatorname{deg} 50^{\prime} 46.7^{\prime \prime}$ & Aug 20 at $02: 10: 37$ (2.6) & 115 \\
\hline $13^{h} 24^{m} 48.72^{s},-32 \operatorname{deg} 14^{\prime} 55.0^{\prime \prime}$ & Aug 20 at $02: 13: 02(2.6)$ & 117 \\
\hline $13^{h} 23^{m} 53.76^{s},-32 \operatorname{deg} 33^{\prime} 42.1^{\prime \prime}$ & Aug 20 at $02: 15: 54$ (2.6) & 122 \\
\hline $13^{h} 25^{m} 22.80^{s},-32 \operatorname{deg} 33^{\prime} 42.1^{\prime \prime}$ & Aug 20 at $02: 18: 24$ (2.6) & 115 \\
\hline $13^{h} 26^{m} 17.76^{s},-32 \operatorname{deg} 14^{\prime} 55.0^{\prime \prime}$ & Aug 20 at $02: 20: 49$ (2.6) & 120 \\
\hline $13^{h} 27^{m} 13.44^{s},-31 \operatorname{deg} 56^{\prime} 07.8^{\prime \prime}$ & Aug 20 at $02: 23: 20$ (2.6) & 122 \\
\hline $13^{h} 28^{m} 10.56^{s},-31 \operatorname{deg} 37^{\prime} 20.6^{\prime \prime}$ & Aug 20 at $02: 25: 50(2.6)$ & 160 \\
\hline $14^{h} 07^{m} 21.12^{s},-49 \operatorname{deg} 09^{\prime} 20.2^{\prime \prime}$ & Aug 20 at 04:09:35 (2.6) & 117 \\
\hline $13^{h} 35^{m} 28.32^{s},-33 \operatorname{deg} 48^{\prime} 50.4^{\prime \prime}$ & Aug 20 at $05: 44: 16(2.7)$ & 17 \\
\hline $14^{h} 04^{m} 40.80^{s},-50 \operatorname{deg} 43^{\prime} 16.0^{\prime \prime}$ & Aug 20 at $05: 45: 34(2.7)$ & 117 \\
\hline $14^{h} 21^{m} 15.60^{s},-54 \operatorname{deg} 47^{\prime} 28.7^{\prime \prime}$ & Aug 20 at $05: 48: 27$ (2.7) & 120 \\
\hline $12^{h} 52^{m} 58.08^{s},-16 \operatorname{deg} 35^{\prime} 38.0^{\prime \prime}$ & Aug 20 at $06: 39: 13(2.7)$ & 100 \\
\hline $13^{h} 16^{m} 36.96^{s},-29 \operatorname{deg} 25^{\prime} 50.5^{\prime \prime}$ & Aug 20 at $06: 41: 42(2.8)$ & 112 \\
\hline $13^{h} 15^{m} 10.80^{s},-29 \operatorname{deg} 25^{\prime} 50.5^{\prime \prime}$ & Aug 20 at $06: 44: 30(2.8)$ & 120 \\
\hline $13^{h} 13^{m} 27.84^{s},-29 \operatorname{deg} 07^{\prime} 03.4^{\prime \prime}$ & Aug 20 at $06: 46: 55(2.8)$ & 125 \\
\hline $13^{h} 14^{m} 53.76^{s},-29 \operatorname{deg} 07^{\prime} 03.4^{\prime \prime}$ & Aug 20 at $06: 49: 25(2.8)$ & 115 \\
\hline $13^{h} 14^{m} 38.40^{s},-28 \operatorname{deg} 48^{\prime} 16.6^{\prime \prime}$ & Aug 20 at $06: 51: 51(2.8)$ & 115 \\
\hline $13^{h} 14^{m} 24.48^{s},-28 \operatorname{deg} 29^{\prime} 29.4^{\prime \prime}$ & Aug 20 at $06: 54: 16(2.8)$ & 120 \\
\hline $13^{h} 14^{m} 12.24^{s},-28 \operatorname{deg} 10^{\prime} 42.2^{\prime \prime}$ & Aug 20 at $06: 56: 40(2.8)$ & 115 \\
\hline $13^{h} 15^{m} 26.40^{s},-27 \operatorname{deg} 51^{\prime} 55.1^{\prime \prime}$ & Aug 20 at $06: 59: 05$ (2.8) & 122 \\
\hline $13^{h} 16^{m} 41.52^{s},-27 \operatorname{deg} 33^{\prime} 07.9^{\prime \prime}$ & Aug 20 at $07: 01: 36(2.8)$ & 120 \\
\hline $13^{h} 17^{m} 57.60^{s},-27 \operatorname{deg} 14^{\prime} 20.8^{\prime \prime}$ & Aug 20 at 07:04:06 (2.8) & 122 \\
\hline $13^{h} 16^{m} 33.12^{s},-27 \operatorname{deg} 14^{\prime} 20.8^{\prime \prime}$ & Aug 20 at 07:06:36 (2.8) & 120 \\
\hline $13^{h} 15^{m} 8.40^{s},-27 \operatorname{deg} 14^{\prime} 20.8^{\prime \prime}$ & Aug 20 at 07:09:02 (2.8) & 115 \\
\hline
\end{tabular}

${ }^{a}$ Observations were 2017, times in parentheses are days since the GW trigger. 


\begin{tabular}{ccc}
\hline & \multicolumn{1}{c}{ Start time $^{a}$} & $\begin{array}{c}\text { Exposure } \\
\text { (UTC) }\end{array}$ \\
(J) $)$
\end{tabular}

${ }^{a}$ Observations were 2017, times in parentheses are days since the GW trigger. 


\begin{tabular}{|c|c|c|}
\hline $\begin{array}{l}\text { Pointing direction } \\
\text { (J2000) }\end{array}$ & $\begin{array}{l}\text { Start time }^{a} \\
\text { (UTC) }\end{array}$ & $\begin{array}{c}\text { Exposure } \\
\text { (s) }\end{array}$ \\
\hline $12^{h} 47^{m} 48.96^{s},-18 \operatorname{deg} 28^{\prime} 21.0^{\prime \prime}$ & Aug 20 at 19:23:57 (3.3) & 22 \\
\hline $12^{h} 49^{m} 8.16^{s},-18 \operatorname{deg} 28^{\prime} 21.0^{\prime \prime}$ & Aug 20 at 19:26:42 (3.3) & 107 \\
\hline $12^{h} 50^{m} 27.36^{s},-18 \operatorname{deg} 28^{\prime} 21.0^{\prime \prime}$ & Aug 20 at 19:29:07 (3.3) & 112 \\
\hline $12^{h} 51^{m} 46.56^{s},-18 \operatorname{deg} 28^{\prime} 21.0^{\prime \prime}$ & Aug 20 at 19:31:32 (3.3) & 120 \\
\hline $12^{h} 51^{m} 12.96^{s},-18 \operatorname{deg} 47^{\prime} 08.2^{\prime \prime}$ & Aug 20 at 19:33:57 (3.3) & 115 \\
\hline $12^{h} 52^{m} 0.00^{s},-19 \operatorname{deg} 05^{\prime} 55.3^{\prime \prime}$ & Aug 20 at 19:36:22 (3.3) & 120 \\
\hline $12^{h} 52^{m} 48.72^{s},-19 \operatorname{deg} 24^{\prime} 42.5^{\prime \prime}$ & Aug 20 at 19:38:47 (3.3) & 120 \\
\hline $12^{h} 52^{m} 19.20^{s},-19 \operatorname{deg} 43^{\prime} 29.6^{\prime \prime}$ & Aug 20 at 19:41:17 (3.3) & 130 \\
\hline $13^{h} 00^{m} 58.56^{s},-14 \operatorname{deg} 24^{\prime} 08.3^{\prime \prime}$ & Aug 20 at 20:59:57 (3.3) & 15 \\
\hline $13^{h} 01^{m} 26.16^{s},-14 \operatorname{deg} 42^{\prime} 55.4^{\prime \prime}$ & Aug 20 at $21: 02: 42$ (3.3) & 102 \\
\hline $13^{h} 01^{m} 55.44^{s},-15 \operatorname{deg} 01^{\prime} 42.6^{\prime \prime}$ & Aug 20 at 21:05:07 (3.4) & 117 \\
\hline $13^{h} 11^{m} 11.28^{s},-27 \operatorname{deg} 51^{\prime} 55.1^{\prime \prime}$ & Aug 20 at $21: 07: 32$ (3.4) & 115 \\
\hline $13^{h} 11^{m} 21.60^{s},-28 \operatorname{deg} 10^{\prime} 42.2^{\prime \prime}$ & Aug 20 at $21: 10: 19(3.4)$ & 117 \\
\hline $13^{h} 09^{m} 46.32^{s},-27 \operatorname{deg} 51^{\prime} 55.1^{\prime \prime}$ & Aug 20 at $21: 12: 43$ (3.4) & 115 \\
\hline $13^{h} 09^{m} 56.40^{s},-28 \operatorname{deg} 10^{\prime} 42.2^{\prime \prime}$ & Aug 20 at $21: 15: 14$ (3.4) & 120 \\
\hline $13^{h} 11^{m} 33.60^{s},-28 \operatorname{deg} 29^{\prime} 29.4^{\prime \prime}$ & Aug 20 at $21: 17: 38$ (3.4) & 125 \\
\hline $13^{h} 13^{m} 12.72^{s},-28 \operatorname{deg} 48^{\prime} 16.6^{\prime \prime}$ & Aug 20 at $21: 20: 10(3.4)$ & 135 \\
\hline $12^{h} 55^{m} 24.24^{s},-20 \operatorname{deg} 21^{\prime} 03.6^{\prime \prime}$ & Aug 20 at $22: 35: 57$ (3.4) & 27 \\
\hline $12^{h} 54^{m} 58.80^{s},-20 \operatorname{deg} 39^{\prime} 50.8^{\prime \prime}$ & Aug 20 at $22: 38: 42(3.4)$ & 110 \\
\hline $12^{h} 54^{m} 34.80^{s},-20 \operatorname{deg} 58^{\prime} 37.9^{\prime \prime}$ & Aug 20 at $22: 41: 06$ (3.4) & 110 \\
\hline $12^{h} 55^{m} 32.88^{s},-21 \operatorname{deg} 17^{\prime} 25.1^{\prime \prime}$ & Aug 20 at $22: 43: 32$ (3.4) & 125 \\
\hline $12^{h} 55^{m} 11.52^{s},-21 \operatorname{deg} 36^{\prime} 12.2^{\prime \prime}$ & Aug 20 at $22: 46: 02(3.4)$ & 117 \\
\hline $12^{h} 56^{m} 32.40^{s},-21 \operatorname{deg} 36^{\prime} 12.2^{\prime \prime}$ & Aug 20 at $22: 48: 27$ (3.4) & 112 \\
\hline $12^{h} 57^{m} 53.28^{s},-21 \operatorname{deg} 36^{\prime} 12.2^{\prime \prime}$ & Aug 20 at $22: 50: 52$ (3.4) & 112 \\
\hline $12^{h} 58^{m} 54.72^{s},-21 \operatorname{deg} 54^{\prime} 59.4^{\prime \prime}$ & Aug 20 at $22: 53: 17$ (3.4) & 125 \\
\hline $13^{h} 00^{m} 34.80^{s},-21 \operatorname{deg} 36^{\prime} 12.2^{\prime \prime}$ & Aug 20 at $22: 55: 48(3.4)$ & 125 \\
\hline $13^{h} 01^{m} 55.68^{s},-21 \operatorname{deg} 36^{\prime} 12.2^{\prime \prime}$ & Aug 20 at $22: 58: 19$ (3.4) & 117 \\
\hline $13^{h} 02^{m} 16.08^{s},-21 \operatorname{deg} 17^{\prime} 25.1^{\prime \prime}$ & Aug 20 at 23:00:44 (3.4) & 120 \\
\hline $12^{h} 46^{m} 29.76^{s},-18 \operatorname{deg} 28^{\prime} 21.0^{\prime \prime}$ & Aug 21 at $00: 10: 57$ (3.5) & 22 \\
\hline $13^{h} 01^{m} 17.28^{s},-20 \operatorname{deg} 58^{\prime} 37.9^{\prime \prime}$ & Aug 21 at $00: 13: 41(3.5)$ & 112 \\
\hline $13^{h} 00^{m} 20.16^{s},-20 \operatorname{deg} 39^{\prime} 50.8^{\prime \prime}$ & Aug 21 at $00: 16: 17$ (3.5) & 112 \\
\hline $12^{h} 58^{m} 59.76^{s},-20 \operatorname{deg} 39^{\prime} 50.8^{\prime \prime}$ & Aug 21 at $00: 18: 48$ (3.5) & 120 \\
\hline $13^{h} 04^{m} 45.12^{s},-20 \operatorname{deg} 21^{\prime} 03.6^{\prime \prime}$ & Aug 21 at $00: 21: 12(3.5)$ & 120 \\
\hline $13^{h} 03^{m} 50.64^{s},-20 \operatorname{deg} 02^{\prime} 16.8^{\prime \prime}$ & Aug 21 at $00: 23: 45(3.5)$ & 122 \\
\hline $13^{h} 06^{m} 18.00^{s},-21 \operatorname{deg} 17^{\prime} 25.1^{\prime \prime}$ & Aug 21 at $00: 26: 15$ (3.5) & 122 \\
\hline $13^{h} 07^{m} 18.96^{s},-21 \operatorname{deg} 36^{\prime} 12.2^{\prime \prime}$ & Aug 21 at 00:28:47 (3.5) & 120 \\
\hline
\end{tabular}

${ }^{a}$ Observations were 2017, times in parentheses are days since the GW trigger. 


\begin{tabular}{ccc}
\hline & \multicolumn{1}{c}{ Start time $^{a}$} & $\begin{array}{c}\text { Exposure } \\
\text { (UTC) }\end{array}$ \\
(J) $)$
\end{tabular}

${ }^{a}$ Observations were 2017, times in parentheses are days since the GW trigger. 


\begin{tabular}{|c|c|c|}
\hline $\begin{array}{l}\text { Pointing direction } \\
\text { (J2000) }\end{array}$ & $\begin{array}{l}\text { Start time }^{a} \\
\text { (UTC) }\end{array}$ & $\begin{array}{c}\text { Exposure } \\
\text { (s) }\end{array}$ \\
\hline $13^{h} 32^{m} 27.36^{s},-33 \operatorname{deg} 48^{\prime} 50.4^{\prime \prime}$ & Aug 21 at 10:10:55 (3.9) & 120 \\
\hline $13^{h} 30^{m} 56.88^{s},-33 \operatorname{deg} 48^{\prime} 50.4^{\prime \prime}$ & Aug 21 at 10:13:20 (3.9) & 120 \\
\hline $13^{h} 30^{m} 15.36^{s},-33 \operatorname{deg} 30^{\prime} 03.2^{\prime \prime}$ & Aug 21 at 10:15:45 (3.9) & 115 \\
\hline $13^{h} 29^{m} 35.52^{s},-33 \operatorname{deg} 11^{\prime} 16.4^{\prime \prime}$ & Aug 21 at 10:18:10 (3.9) & 120 \\
\hline $13^{h} 28^{m} 57.60^{s},-32 \operatorname{deg} 52^{\prime} 29.3^{\prime \prime}$ & Aug 21 at 10:20:35 (3.9) & 120 \\
\hline $13^{h} 28^{m} 5.76^{s},-33 \operatorname{deg} 11^{\prime} 16.4^{\prime \prime}$ & Aug 21 at 10:23:00 (3.9) & 117 \\
\hline $13^{h} 37^{m} 2.88^{s},-39 \operatorname{deg} 45^{\prime} 46.1^{\prime \prime}$ & Aug 21 at 10:25:25 (3.9) & 117 \\
\hline $14^{h} 01^{m} 54.00^{s},-53 \operatorname{deg} 32^{\prime} 20.0^{\prime \prime}$ & Aug 21 at 10:28:04 (3.9) & 117 \\
\hline $12^{h} 51^{m} 50.88^{s},-20 \operatorname{deg} 02^{\prime} 16.8^{\prime \prime}$ & Aug 21 at 11:20:57 (3.9) & 17 \\
\hline $12^{h} 52^{m} 43.92^{s},-20 \operatorname{deg} 21^{\prime} 03.6^{\prime \prime}$ & Aug 21 at $11: 23: 42$ (3.9) & 117 \\
\hline $12^{h} 53^{m} 38.64^{s},-20 \operatorname{deg} 39^{\prime} 50.8^{\prime \prime}$ & Aug 21 at $11: 26: 12$ (3.9) & 115 \\
\hline $12^{h} 52^{m} 18.24^{s},-20 \operatorname{deg} 39^{\prime} 50.8^{\prime \prime}$ & Aug 21 at $11: 28: 43$ (3.9) & 115 \\
\hline $12^{h} 50^{m} 58.08^{s},-20 \operatorname{deg} 39^{\prime} 50.8^{\prime \prime}$ & Aug 21 at $11: 31: 08(4.0)$ & 112 \\
\hline $13^{h} 10^{m} 44.40^{s},-26 \operatorname{deg} 36^{\prime} 46.4^{\prime \prime}$ & Aug 21 at $11: 33: 33$ (4.0) & 117 \\
\hline $13^{h} 10^{m} 48.96^{s},-26 \operatorname{deg} 55^{\prime} 33.6^{\prime \prime}$ & Aug 21 at $11: 36: 13$ (4.0) & 117 \\
\hline $13^{h} 09^{m} 20.40^{s},-26 \operatorname{deg} 36^{\prime} 46.4^{\prime \prime}$ & Aug 21 at $11: 38: 38$ (4.0) & 125 \\
\hline $13^{h} 10^{m} 41.28^{s},-26 \operatorname{deg} 17^{\prime} 59.3^{\prime \prime}$ & Aug 21 at $11: 41: 08(4.0)$ & 120 \\
\hline $13^{h} 12^{m} 5.04^{s},-26 \operatorname{deg} 17^{\prime} 59.3^{\prime \prime}$ & Aug 21 at $11: 43: 39(4.0)$ & 117 \\
\hline $13^{h} 13^{m} 29.04^{s},-26 \operatorname{deg} 17^{\prime} 59.3^{\prime \prime}$ & Aug 21 at $11: 46: 04$ (4.0) & 120 \\
\hline $13^{h} 14^{m} 52.80^{s},-26 \operatorname{deg} 17^{\prime} 59.3^{\prime \prime}$ & Aug 21 at $11: 48: 29(4.0)$ & 117 \\
\hline $13^{h} 16^{m} 13.92^{s},-25 \operatorname{deg} 59^{\prime} 12.1^{\prime \prime}$ & Aug 21 at $11: 50: 54$ (4.0) & 120 \\
\hline $13^{h} 16^{m} 12.96^{s},-25 \operatorname{deg} 40^{\prime} 25.0^{\prime \prime}$ & Aug 21 at 11:53:24 (4.0) & 112 \\
\hline $13^{h} 14^{m} 49.44^{s},-25 \operatorname{deg} 40^{\prime} 25.0^{\prime \prime}$ & Aug 21 at $11: 55: 48$ (4.0) & 120 \\
\hline $13^{h} 17^{m} 36.24^{s},-25 \operatorname{deg} 40^{\prime} 25.0^{\prime \prime}$ & Aug 21 at $11: 58: 14(4.0)$ & 122 \\
\hline $13^{h} 36^{m} 23.76^{s},-35 \operatorname{deg} 22^{\prime} 46.2^{\prime \prime}$ & Aug 21 at 12:00:45 (4.0) & 117 \\
\hline $14^{h} 00^{m} 36.48^{s},-48 \operatorname{deg} 31^{\prime} 45.8^{\prime \prime}$ & Aug 21 at 12:03:29 (4.0) & 117 \\
\hline $13^{h} 31^{m} 56.40^{s},-32 \operatorname{deg} 52^{\prime} 29.3^{\prime \prime}$ & Aug 21 at 13:00:09 (4.0) & 87 \\
\hline $13^{h} 31^{m} 19.44^{s},-32 \operatorname{deg} 33^{\prime} 42.1^{\prime \prime}$ & Aug 21 at 13:02:30 (4.0) & 117 \\
\hline $13^{h} 32^{m} 48.72^{s},-32 \operatorname{deg} 33^{\prime} 42.1^{\prime \prime}$ & Aug 21 at 13:04:55 (4.0) & 112 \\
\hline $13^{h} 33^{m} 25.92^{s},-32 \operatorname{deg} 52^{\prime} 29.3^{\prime \prime}$ & Aug 21 at 13:07:20 (4.0) & 112 \\
\hline $13^{h} 34^{m} 17.76^{s},-32 \operatorname{deg} 33^{\prime} 42.1^{\prime \prime}$ & Aug 21 at 13:09:44 (4.0) & 120 \\
\hline $13^{h} 34^{m} 55.44^{s},-32 \operatorname{deg} 52^{\prime} 29.3^{\prime \prime}$ & Aug 21 at $13: 12: 10(4.0)$ & 115 \\
\hline $13^{h} 35^{m} 34.80^{s},-33 \operatorname{deg} 11^{\prime} 16.4^{\prime \prime}$ & Aug 21 at $13: 14: 35$ (4.0) & 112 \\
\hline $13^{h} 36^{m} 24.96^{s},-32 \operatorname{deg} 52^{\prime} 29.3^{\prime \prime}$ & Aug 21 at 13:17:00 (4.0) & 120 \\
\hline $13^{h} 37^{m} 54.48^{s},-32 \operatorname{deg} 52^{\prime} 29.3^{\prime \prime}$ & Aug 21 at 13:19:25 (4.0) & 115 \\
\hline $13^{h} 37^{m} 4.56^{s},-33 \operatorname{deg} 11^{\prime} 16.4^{\prime \prime}$ & Aug 21 at 13:21:50 (4.0) & 117 \\
\hline
\end{tabular}

${ }^{a}$ Observations were 2017, times in parentheses are days since the GW trigger. 


\begin{tabular}{|c|c|c|}
\hline $\begin{array}{l}\text { Pointing direction } \\
\text { (J2000) }\end{array}$ & $\begin{array}{l}\text { Start time }^{a} \\
\text { (UTC) }\end{array}$ & $\begin{array}{c}\text { Exposure } \\
\text { (s) }\end{array}$ \\
\hline $13^{h} 37^{m} 46.08^{s},-33 \operatorname{deg} 30^{\prime} 03.2^{\prime \prime}$ & Aug 21 at 13:24:15 (4.0) & 117 \\
\hline $13^{h} 38^{m} 29.28^{s},-33 \operatorname{deg} 48^{\prime} 50.4^{\prime \prime}$ & Aug 21 at 13:26:40 (4.0) & 112 \\
\hline $13^{h} 39^{m} 59.52^{s},-33 \operatorname{deg} 48^{\prime} 50.4^{\prime \prime}$ & Aug 21 at 13:29:05 (4.0) & 117 \\
\hline $13^{h} 40^{m} 46.32^{s},-33 \operatorname{deg} 30^{\prime} 03.2^{\prime \prime}$ & Aug 21 at 13:31:30 (4.0) & 117 \\
\hline $13^{h} 39^{m} 16.08^{s},-33 \operatorname{deg} 30^{\prime} 03.2^{\prime \prime}$ & Aug 21 at 13:33:55 (4.0) & 115 \\
\hline $13^{h} 40^{m} 6.24^{s},-36 \operatorname{deg} 37^{\prime} 54.8^{\prime \prime}$ & Aug 21 at 13:36:20 (4.0) & 115 \\
\hline $13^{h} 50^{m} 45.36^{s},-48 \operatorname{deg} 12^{\prime} 58.7^{\prime \prime}$ & Aug 21 at 13:38:54 (4.0) & 117 \\
\hline $13^{h} 30^{m} 9.36^{s},-34 \operatorname{deg} 45^{\prime} 11.9^{\prime \prime}$ & Aug 21 at 14:36:07 (4.1) & 87 \\
\hline $13^{h} 30^{m} 57.36^{s},-35 \operatorname{deg} 03^{\prime} 59.0^{\prime \prime}$ & Aug 21 at 14:38:32 (4.1) & 117 \\
\hline $13^{h} 32^{m} 29.28^{s},-35 \operatorname{deg} 03^{\prime} 59.0^{\prime \prime}$ & Aug 21 at 14:40:57 (4.1) & 115 \\
\hline $13^{h} 33^{m} 12.24^{s},-34 \operatorname{deg} 45^{\prime} 11.9^{\prime \prime}$ & Aug 21 at 14:43:22 (4.1) & 120 \\
\hline $13^{h} 32^{m} 25.44^{s},-34 \operatorname{deg} 26^{\prime} 24.7^{\prime \prime}$ & Aug 21 at 14:45:47 (4.1) & 120 \\
\hline $13^{h} 30^{m} 54.24^{s},-34 \operatorname{deg} 26^{\prime} 24.7^{\prime \prime}$ & Aug 21 at 14:48:12 (4.1) & 115 \\
\hline $13^{h} 31^{m} 40.32^{s},-34 \operatorname{deg} 07^{\prime} 37.6^{\prime \prime}$ & Aug 21 at 14:50:37 (4.1) & 115 \\
\hline $13^{h} 27^{m} 7.92^{s},-34 \operatorname{deg} 07^{\prime} 37.6^{\prime \prime}$ & Aug 21 at 14:53:02 (4.1) & 117 \\
\hline $13^{h} 25^{m} 37.20^{s},-34 \operatorname{deg} 07^{\prime} 37.6^{\prime \prime}$ & Aug 21 at 14:55:33 (4.1) & 112 \\
\hline $13^{h} 24^{m} 55.20^{s},-33 \operatorname{deg} 48^{\prime} 50.4^{\prime \prime}$ & Aug 21 at 14:57:58 (4.1) & 115 \\
\hline $13^{h} 24^{m} 14.88^{s},-33 \operatorname{deg} 30^{\prime} 03.2^{\prime \prime}$ & Aug 21 at 15:00:22 (4.1) & 120 \\
\hline $13^{h} 25^{m} 6.24^{s},-33 \operatorname{deg} 11^{\prime} 16.4^{\prime \prime}$ & Aug 21 at 15:02:48 (4.1) & 117 \\
\hline $13^{h} 25^{m} 58.56^{s},-32 \operatorname{deg} 52^{\prime} 29.3^{\prime \prime}$ & Aug 21 at 15:05:13 (4.1) & 120 \\
\hline $13^{h} 27^{m} 28.08^{s},-32 \operatorname{deg} 52^{\prime} 29.3^{\prime \prime}$ & Aug 21 at 15:07:38 (4.1) & 120 \\
\hline $13^{h} 28^{m} 21.12^{s},-32 \operatorname{deg} 33^{\prime} 42.1^{\prime \prime}$ & Aug 21 at 15:10:03 (4.1) & 115 \\
\hline $13^{h} 38^{m} 20.40^{s},-40 \operatorname{deg} 04^{\prime} 33.2^{\prime \prime}$ & Aug 21 at $15: 12: 27$ (4.1) & 117 \\
\hline $14^{h} 06^{m} 10.56^{s},-55 \operatorname{deg} 25^{\prime} 03.0^{\prime \prime}$ & Aug 21 at 15:15:08 (4.1) & 117 \\
\hline $13^{h} 07^{m} 54.24^{s},-19 \operatorname{deg} 05^{\prime} 55.3^{\prime \prime}$ & Aug 21 at 16:08:57 (4.1) & 17 \\
\hline $13^{h} 08^{m} 24.72^{s},-18 \operatorname{deg} 47^{\prime} 08.2^{\prime \prime}$ & Aug 21 at $16: 11: 41(4.1)$ & 105 \\
\hline $13^{h} 07^{m} 37.20^{s},-18 \operatorname{deg} 28^{\prime} 21.0^{\prime \prime}$ & Aug 21 at $16: 14: 07$ (4.1) & 120 \\
\hline $13^{h} 06^{m} 18.00^{s},-18 \operatorname{deg} 28^{\prime} 21.0^{\prime \prime}$ & Aug 21 at $16: 16: 31$ (4.1) & 120 \\
\hline $13^{h} 06^{m} 51.36^{s},-18 \operatorname{deg} 09^{\prime} 33.8^{\prime \prime}$ & Aug 21 at $16: 18: 57$ (4.2) & 117 \\
\hline $13^{h} 12^{m} 24.72^{s},-21 \operatorname{deg} 54^{\prime} 59.4^{\prime \prime}$ & Aug 21 at $16: 21: 22(4.2)$ & 117 \\
\hline $13^{h} 10^{m} 47.28^{s},-22 \operatorname{deg} 13^{\prime} 46.6^{\prime \prime}$ & Aug 21 at $16: 23: 57$ (4.2) & 122 \\
\hline $13^{h} 11^{m} 53.52^{s},-22 \operatorname{deg} 32^{\prime} 33.7^{\prime \prime}$ & Aug 21 at $16: 26: 28$ (4.2) & 122 \\
\hline $13^{h} 13^{m} 1.44^{s},-22 \operatorname{deg} 51^{\prime} 20.9^{\prime \prime}$ & Aug 21 at $16: 28: 58$ (4.2) & 120 \\
\hline $13^{h} 14^{m} 11.04^{s},-23 \operatorname{deg} 10^{\prime} 08.0^{\prime \prime}$ & Aug 21 at 16:31:29 (4.2) & 115 \\
\hline $13^{h} 14^{m} 0.48^{s},-23 \operatorname{deg} 28^{\prime} 55.2^{\prime \prime}$ & Aug 21 at 16:33:59 (4.2) & 112 \\
\hline $13^{h} 15^{m} 13.68^{s},-23 \operatorname{deg} 47^{\prime} 42.4^{\prime \prime}$ & Aug 21 at 16:36:24 (4.2) & 117 \\
\hline
\end{tabular}

${ }^{a}$ Observations were 2017, times in parentheses are days since the GW trigger. 


\begin{tabular}{clc}
\hline $\begin{array}{c}\text { Pointing direction } \\
(\mathrm{J} 2000)\end{array}$ & $\begin{array}{c}\text { Start time } \\
(\mathrm{UTC})\end{array}$ & $\begin{array}{c}\text { Exposure } \\
(\mathrm{s})\end{array}$ \\
\hline $13^{h} 16^{m} 28.32^{s},-24 \operatorname{deg} 06^{\prime} 29.5^{\prime \prime}$ & Aug 21 at 16:38:55(4.2) & 125 \\
$13^{h} 16^{m} 22.56^{s},-24 \operatorname{deg} 25^{\prime} 16.7^{\prime \prime}$ & Aug 21 at 16:41:25(4.2) & 115 \\
$13^{h} 14^{m} 55.20^{s},-24 \operatorname{deg} 44^{\prime} 03.5^{\prime \prime}$ & Aug 21 at 16:43:50(4.2) & 122 \\
$13^{h} 29^{m} 15.36^{s},-32 \operatorname{deg} 14^{\prime} 55.0^{\prime \prime}$ & Aug 21 at 16:46:21(4.2) & 117 \\
$13^{h} 47^{m} 29.76^{s},-39 \operatorname{deg} 08^{\prime} 11.8^{\prime \prime}$ & Aug 21 at 16:49:02(4.2) & 117 \\
$14^{h} 08^{m} 23.04^{s},-55 \operatorname{deg} 25^{\prime} 03.0^{\prime \prime}$ & Aug 21 at 16:51:42(4.2) & 140 \\
$13^{h} 03^{m} 25.44^{s},-16 \operatorname{deg} 35^{\prime} 38.0^{\prime \prime}$ & Aug 21 at 17:43:57(4.2) & 17 \\
$13^{h} 02^{m} 48.96^{s},-16 \operatorname{deg} 16^{\prime} 50.9^{\prime \prime}$ & Aug 21 at 17:46:42(4.2) & 107 \\
$13^{h} 14^{m} 51.84^{s},-25 \operatorname{deg} 02^{\prime} 50.6^{\prime \prime}$ & Aug 21 at 17:49:07(4.2) & 115 \\
$13^{h} 16^{m} 18.00^{s},-24 \operatorname{deg} 44^{\prime} 03.5^{\prime \prime}$ & Aug 21 at 17:51:49(4.2) & 117 \\
$13^{h} 17^{m} 37.68^{s},-25 \operatorname{deg} 02^{\prime} 50.6^{\prime \prime}$ & Aug 21 at 17:54:20(4.2) & 117 \\
$13^{h} 17^{m} 40.80^{s},-24 \operatorname{deg} 44^{\prime} 03.5^{\prime \prime}$ & Aug 21 at 17:56:50(4.2) & 117 \\
$13^{h} 19^{m} 7.44^{s},-24 \operatorname{deg} 25^{\prime} 16.7^{\prime \prime}$ & Aug 21 at 17:59:15(4.2) & 122 \\
$13^{h} 31^{m} 39.12^{s},-31 \operatorname{deg} 56^{\prime} 07.8^{\prime \prime}$ & Aug 21 at 18:22:42(4.2) & 117 \\
$13^{h} 54^{m} 55.92^{s},-48 \operatorname{deg} 31^{\prime} 45.8^{\prime \prime}$ & Aug 21 at 18:25:23(4.2) & 117 \\
$12^{h} 57^{m} 15.36^{s},-22 \operatorname{deg} 13^{\prime} 46.6^{\prime \prime}$ & Aug 21 at 19:19:57(4.3) & 22 \\
$12^{h} 56^{m} 12.72^{s},-21 \operatorname{deg} 54^{\prime} 59.4^{\prime \prime}$ & Aug 21 at 19:22:42(4.3) & 107 \\
$13^{h} 07^{m} 57.12^{s},-25 \operatorname{deg} 02^{\prime} 50.6^{\prime \prime}$ & Aug 21 at 19:25:11(4.3) & 120 \\
$13^{h} 06^{m} 38.88^{s},-24 \operatorname{deg} 44^{\prime} 03.5^{\prime \prime}$ & Aug 21 at 19:27:48 (4.3) & 122 \\
$13^{h} 05^{m} 16.08^{s},-24 \operatorname{deg} 44^{\prime} 03.5^{\prime \prime}$ & Aug 21 at 19:30:18(4.3) & 120 \\
$13^{h} 05^{m} 11.28^{s},-25 \operatorname{deg} 02^{\prime} 50.6^{\prime \prime}$ & Aug 21 at 19:32:43(4.3) & 115 \\
$13^{h} 03^{m} 53.28^{s},-24 \operatorname{deg} 44^{\prime} 03.5^{\prime \prime}$ & Aug 21 at 19:35:08 (4.3) & 142 \\
\hline
\end{tabular}

${ }^{a}$ Observations were 2017, times in parentheses are days since the GW trigger. 
24. A. J. van der Horst, et al., Astrophys. J 699, 1087 (2009).

25. T. LVC, et al., ApJL, submitted .

26. O. Gottlieb, E. Nakar, T. Piran, ArXiv 1705.10797 (2017).

27. D. Lazzati, A. Deich, B. J. Morsony, J. C. Workman, Mon. Not. R. Astron. Soc 471, 1652 (2017).

28. A. Kathirgamaraju, R. Barniol Duran, D. Giannios, ArXiv 1708.07488 (2017).

29. D. Malesani, D. Watson, J. Hjorth, Gamma Ray Coordinates Network Circular 21577 (2017).

30. P. Cowperthwaite, M. Nicholl, E. Berger, Gamma Ray Coordinates Network Circular 21578 (2017).

31. M. Modjaz, Y. Q. Liu, F. B. Bianco, O. Graur, Astrophys. J 832, 108 (2016).

32. P. S. Cowperthwaite, E. Berger, Astrophys. J 814, 25 (2015).

33. L.-X. Li, B. Paczyński, Astrophys. J. Lett. 507, L59 (1998).

34. B. D. Metzger, Living Reviews in Relativity 20, 3 (2017).

35. S. Rosswog, et al., Astron. Astrophys. 341, 499 (1999).

36. B. D. Metzger, A. L. Piro, E. Quataert, Mon. Not. R. Astron. Soc 396, 304 (2009).

37. A. Perego, et al., Mon. Not. R. Astron. Soc 443, 3134 (2014).

38. D. Martin, et al., Astrophys. J 813, 2 (2015).

39. O. Just, A. Bauswein, R. A. Pulpillo, S. Goriely, H.-T. Janka, Mon. Not. R. Astron. Soc 448, 541 (2015).

40. D. Kasen, N. R. Badnell, J. Barnes, Astrophys. J 774, 25 (2013).

41. J. Barnes, D. Kasen, Astrophys. J 775, 18 (2013).

42. E. M. Burbidge, G. R. Burbidge, W. A. Fowler, F. Hoyle, Reviews of Modern Physics 29, 547 (1957).

43. R. Fernández, D. Kasen, B. D. Metzger, E. Quataert, Mon. Not. R. Astron. Soc 446, 750 (2015).

44. R. T. Wollaeger, et al., ArXiv 1705.07084 (2017).

45. S. Rosswog, O. Korobkin, A. Arcones, F.-K. Thielemann, T. Piran, Mon. Not. R. Astron. Soc 439, 744 (2014).

46. E. Troja, L. Piro, T. Sakamoto, B. Cenko, A. Lien, Gamma Ray Coordinates Network Circular 21765 (2017).

47. E. Troja, et al., Nature, DOI: 10.1038/nature24290 .

48. D. Haggard, et al., ApJL, DOI: 0.3847/2041-8213/aa8ede 848.

49. J. E. Rhoads, Astrophys. J 525, 737 (1999).

50. P. A. Evans, et al., Mon. Not. R. Astron. Soc 455, 1522 (2016).

51. D. Eichler, M. Livio, T. Piran, D. N. Schramm, Nature 340, 126 (1989). 
52. N. R. Tanvir, et al., Nature 500, 547 (2013).

53. E. Berger, W. Fong, R. Chornock, Astrophys. J. Lett. 774, L23 (2013).

54. A. von Kienlin, C. Meegan, A. Goldstein, Gamma Ray Coordinates Network Circular 21520 (2017).

55. V. Savchenko, et al., Gamma Ray Coordinates Network Circular 21507 (2017).

56. S. D. Barthelmy, et al., Space Sci. Rev. 120, 143 (2005).

57. D. N. Burrows, et al., Space Sci. Rev. 120, 165 (2005).

58. P. W. A. Roming, et al., Space Sci. Rev. 120, 95 (2005).

59. The LIGO Scientific Collaboration, the Virgo Collaboration, Gamma Ray Coordinates Network Circular 21509 (2017).

60. D. J. White, E. J. Daw, V. S. Dhillon, Class. Quantum Gravity 28, 085016 (2011).

61. W. L. Freedman, et al., Astrophys. J 553, 47 (2001).

62. S. Allam, et al., Gamma Ray Coordinates Network Circular 21530 (2017).

63. S. Yang, et al., Gamma Ray Coordinates Network Circular 21531 (2017).

64. P. Evans, et al., Gamma Ray Coordinates Network Circular 21550 (2017).

65. A. Lien, et al., Astrophys. J 829, 7 (2016).

66. https://heasarc.gsfc.nasa.gov/lheasoft/ .

67. https://heasarc.gsfc.nasa.gov/docs/heasarc/caldb/ .

68. T. S. Poole, et al., Mon. Not. R. Astron. Soc 383, 627 (2008).

69. A. A. Breeveld, et al., American Institute of Physics Conference Series, J. E. McEnery, J. L. Racusin, N. Gehrels, eds. (2011), vol. 1358 of American Institute of Physics Conference Series, pp. 373-376.

70. E. F. Schlafly, D. P. Finkbeiner, Astrophys. J 737, 103 (2011).

71. P. A. Evans, et al., Astrophys. J. Suppl. Ser. 210, 8 (2014).

72. P. A. Evans, et al., Mon. Not. R. Astron. Soc 460, L40 (2016).

73. P. A. Evans, et al., Mon. Not. R. Astron. Soc 397, 1177 (2009).

74. P. Evans, et al., Gamma Ray Coordinates Network Circular 21612 (2017).

75. R. Margutti, et al., Gamma Ray Coordinates Network Circular 21648 (2017).

76. R. Margutti, et al., The Astrophysical Journal Letters, DOI: 10.3847/2041-8213/aa9057 848.

77. K. Alexander, W. Fong, E. Berger, Gamma Ray Coordinates Network Circular 21548 (2017). 
78. K. Bannister, C. Lynch, D. Kaplan, T. Murphy, D. Dobie, Gamma Ray Coordinates Network Circular 21559 (2017).

79. K. Alexander, et al., The Astrophysical Journal Letters, DOI: 10.3847/2041-8213/aa905d 848.

80. D. R. Wik, et al., Astrophys. J 792, 48 (2014).

81. A. Goldstein, et al., Gamma Ray Coordinates Network Circular 21528 (2017).

82. K. Chambers, et al., Gamma Ray Coordinates Network Circular 21617 (2017).

83. R. T. Wollaeger, et al., Astrophys. J. Supplement 209, 36 (2013).

84. R. T. Wollaeger, D. R. van Rossum 214, 28 (2014).

85. D. R. van Rossum, et al., Astrophys. J 827, 128 (2016).

86. C. J. Fontes, et al., High Energy Density Physics 16, 53 (2015).

87. C. J. Fontes, et al., Journal of Physics B: Atomic, Molecular and Optical Physics 48, 144014 (2015).

88. C. J. Fontes, et al., ArXiv 1702.02990 (2017).

89. S. Rosswog, Philosophical Transactions of the Royal Society of London Series A 371, 20272 (2013).

90. C. Winteler, Light Element Production in the Big Bang and the Synthesis of Heavy Elements in 3D MHD Jets from Core-Collapse Supernovae, Ph.D. thesis, The University of Basel, Basel, Switzerland (2012).

91. O. Korobkin, S. Rosswog, A. Arcones, C. Winteler, Mon. Not. R. Astron. Soc 426, 1940 (2012).

92. F.-K. Thielemann, et al., Progress in Particle and Nuclear Physics 66, 346 (2011).

93. T. Rauscher, F.-K. Thielemann, Atomic Data and Nuclear Data Tables 75, 1 (2000).

94. P. Möller, J. R. Nix, W. D. Myers, W. J. Swiatecki, Atomic Data and Nuclear Data Tables 59, 185 (1995).

95. A. Arcones, G. Martínez-Pinedo, Phys. Review C 83, 045809 (2011).

96. I. V. Panov, et al., Astron. \& Astrophys. 513, A61 (2010).

97. I. V. Panov, et al., Nuclear Physics A 747, 633 (2005).

98. J. Barnes, D. Kasen, M.-R. Wu, G. Martínez-Pinedo, Astrophys. J 829, 110 (2016).

99. S. Rosswog, et al., Classical and Quantum Gravity 34, 104001 (2017).

100. D. Grossman, O. Korobkin, S. Rosswog, T. Piran, Mon. Not. R. Astron. Soc 439, 757 (2014).

101. A. Perego, et al., Mon. Not. R. Astron. Soc 443, 3134 (2014).

102. D. M. Siegel, B. D. Metzger, ArXiv 1705.05473 (2017).

103. R. Sari, T. Piran, R. Narayan, Astrophys. J. Lett. 497, L17 (1998). 
104. J. Granot, R. Sari, Astrophys. J 568, 820 (2002).

105. E. Troja, L. Piro, T. Sakamoto, B. Cenko, A. Lien, Gamma Ray Coordinates Network Circular 21787 (2017).

106. D. Haggard, M. Nynka, V. Kalogera, P. Evans, S. Cenko, Gamma Ray Coordinates Network Circular 21798 (2017).

107. K. Mooley, G. Hallinan, A. Corsi, Gamma Ray Coordinates Network Circular 21814 (2017).

108. A. Corsi, et al., Gamma Ray Coordinates Network Circular 21815 (2017).

109. D. Lazzati, et al., ArXiv 1709.01468 (2017).

110. S. Kisaka, K. Ioka, E. Nakar, Astrophys. J 818, 104 (2016).

111. W. Fong, et al., Astrophys. J 780, 118 (2014).

112. N. R. Tanvir, et al., Gamma Ray Coordinates Network Circular 15489, 1 (2013).

113. W. Fong, R. Margutti, D. Haggard, Gamma Ray Coordinates Network Circular 21786 (2017).

114. A. Rowlinson, P. T. O’Brien, B. D. Metzger, N. R. Tanvir, A. J. Levan, Mon. Not. R. Astron. Soc 430, 1061 (2013).

115. B. P. Gompertz, P. T. O’Brien, G. A. Wynn, A. Rowlinson, Mon. Not. R. Astron. Soc 431, 1745 (2013).

116. B. P. Gompertz, A. J. van der Horst, P. T. O’Brien, G. A. Wynn, K. Wiersema, Mon. Not. R. Astron. Soc 448, 629 (2015).

117. H. Sun, B. Zhang, H. Gao, Astrophys. J 835, 7 (2017).

118. D. M. Siegel, R. Ciolfi, Astrophys. J 819, 15 (2016).

119. K. Hotokezaka, et al., Mon. Not. R. Astron. Soc 459, 35 (2016).

120. R. Foley, Gamma Ray Coordinates Network Circular 21557 (2017). 